\title{
A Quantitative Examination of the Effects of Silicon Substituents on the Efficiency of Cross-Coupling Reactions
}

\author{
Scott E. Denmark*, Luc Neuville, Matthew E. L. Christy and Steven A. Tymonko \\ Roger Adams Laboratory, Department of Chemistry \\ University of Illinois, Urbana, Illinois 61801
}

\section{SUPPORTING INFORMATION}

Table of Contents

Page

General Experimental

Literature Preparations

Experimental Procedures

Preparation of Alkenylsilane Precursors

Preparation of Reference Compounds

Preparation of $\mathbf{2 4}$

Determination of Response Factors

Competition Experiments, GC Data

NMR Spectra

\section{General Experimental}

All reactions were performed in oven-dried $\left(140^{\circ} \mathrm{C}\right)$ or flame-dried glassware under an inert atmosphere of dry $\mathrm{N}_{2}$. The following reaction solvents were distilled from the indicated drying agents: diethyl ether (sodium, benzophenone), toluene $(\mathrm{Na})$, methanol $\left(\mathrm{Mg}(\mathrm{OMe})_{2}\right)$, triethylamine $\left(\mathrm{CaH}_{2}\right)$, tert-butyl alcohol was distilled over Na. n-Butyllithium solutions were titrated following the method of Gilman. ${ }^{1}$ Brine refers to a sat. aq. solution of $\mathrm{NaCl}$.

${ }^{1} \mathrm{H}$ NMR, ${ }^{13} \mathrm{C}$ NMR and ${ }^{19} \mathrm{~F}$ NMR were recorded on $500 \mathrm{MHz},{ }^{1} \mathrm{H} ; 470 \mathrm{MHz},{ }^{19} \mathrm{~F} ; 126$ $\mathrm{MHz},{ }^{13} \mathrm{C}$ spectrometers. Spectra were referenced to residual chloroform $\left(7.26 \mathrm{ppm},{ }^{1} \mathrm{H} ; 77.00\right.$ $\left.\mathrm{ppm},{ }^{13} \mathrm{C}\right)$. Chemical shifts are reported in ppm ( $\delta$ ); multiplicities are indicated by s (singlet), $\mathrm{d}$ 
(doublet), t (triplet), q (quartet), qn (quintet), sept (septet), m (multiplet) and br (broad). Coupling constants, $J$, are reported in Hertz. ${ }^{1} \mathrm{H}$ and ${ }^{13} \mathrm{C}$ NMR assignments are corroborated by 2D experiments (HETCOR and COSY). Spectra are available on request from denmark@scs.uiuc.edu. Mass spectroscopy data (EI, CI, FAB) are reported in the form of (m/z). Infrared spectra (IR) were recorded in $\mathrm{NaCl}$ cells and peaks are reported in $\mathrm{cm}^{-1}$ with indicated relative intensities: s (strong, 67-100\%); $\mathrm{m}$ (medium, 34-66\%); w (weak, 0-33\%).

Analytical thin-layer chromatography was performed on silica or aluminum oxide, basic gel plates with QF-254 indicator. Visualization was accomplished with $\mathrm{KMnO}_{4}$, UV light and/or iodine. Diethyl ether was of reagent grade and used as received; other solvents for chromatography and filtration were technical grade and distilled from the indicated drying agents: hexane and pentane $\left(\mathrm{CaCl}_{2}\right)$; ethyl acetate $\left(\mathrm{K}_{2} \mathrm{CO}_{3}\right)$; dichloromethane $\left(\mathrm{CaCl}_{2}\right)$. Column chromatography was performed using 230-400-mesh silica.

Analytical capillary gas chromatography (GC) was performed using a gas chromatograph fitted with a flame ionization detector $\left(\mathrm{H}_{2}\right.$ carrier gas, $\left.1 \mathrm{~mL} / \mathrm{min}\right)$ : The following column was used: HP-5 50-m cross-linked 5\%-phenyl methyl silicone gum phase. The detector temperature was $300{ }^{\circ} \mathrm{C}$. Retention times $\left(t_{R}\right)$ and integrated ratios were obtained from a reporting integrator. Retention times (HP 5, $250{ }^{\circ} \mathrm{C}, 15 \mathrm{psi}$ ): $t_{R}$ naphthalene, $4.84 \mathrm{~min} ; t_{R} \mathbf{1 2}, 6.43 \mathrm{~min} ; t_{R} \mathbf{1 3}, 8.30$ $\min ; t_{R} \mathbf{1 4}, 4.94 \mathrm{~min} ; t_{R} \mathbf{1 5}, 5.60 \mathrm{~min} ; t_{R} \mathbf{1 6}, 5.52 \mathrm{~min} ; t_{R} \mathbf{1 7}, 6.65 \mathrm{~min}$. Retention times (HP 5, $\left.200{ }^{\circ} \mathrm{C}, 15 \mathrm{psi}\right): t_{R}$ naphthalene, $5.48 \mathrm{~min} ; t_{R} \mathbf{1 4}, 6.06 \mathrm{~min} ; t_{R} \mathbf{1 5}, 8.47 \mathrm{~min}$.

Bulb-to-bulb distillations were performed on a Kugelrohr, boiling points (bp) corresponding to uncorrected air-bath temperatures (ABT). Commercial reagents were purified by distillation or recrystallization prior to use. A $1.0 \mathrm{M}$ solution of tetrabutylammonium fluoride in THF was prepared from solid tetrabutylammonium fluoride trihydrate $\left(\mathrm{TBAF} \bullet 3 \mathrm{H}_{2} \mathrm{O}\right.$, Fluka) and distilled THF in a volumetric flask and was stored in a Schlenk bottle. A solution of THF containing TBAF $\left(\begin{array}{lll}1.0 & \mathrm{M}\end{array}\right)$ and naphthalene $\left(\begin{array}{lll}0.25 & \mathrm{M}\end{array}\right)$ was prepared from solid tetrabutylammonium fluoride trihydrate $\left(\mathrm{TBAF} \bullet 3 \mathrm{H}_{2} \mathrm{O}\right.$, Fluka) or naphthalene and distilled THF in a volumetric flask and was stored in a Schlenk bottle. Palladium bis(dibenzylideneacetone) $\left(\operatorname{Pd}(\mathrm{dba})_{2}\right)$ and allylpalladium chloride dimer were used without purification. The $t-\mathrm{Bu} 3 \mathrm{P}-\mathrm{Pt}(0)-$ DVDS complex was prepared by the literature procedure ${ }^{2}: t-\mathrm{Bu}_{3} \mathrm{P}(32 \mathrm{mg}, 0.158 \mathrm{mmol})$ was dissolved in platinum(0)-1,3-divinyl-1,1,3,3-tetramethyldisiloxane complex in xylene $(1.5 \mathrm{~mL}$ 
xylene solution). The mixture was stirred at $65{ }^{\circ} \mathrm{C}$ (oil bath) for $5 \mathrm{~min}$ and then was slowly cooled to room temperature. This solution could be stored under nitrogen in the freezer $\left(-20{ }^{\circ} \mathrm{C}\right)$ indefinitely.

The following compounds were prepared by literature methods. (E)-1-iodo-1-heptene, ${ }^{3}$ $(E)$-1-iodo-1-pentene, ${ }^{4} \quad(E)$-dimethyl-(1-heptenyl)silanol ${ }^{3} \quad(2), \quad(E)$-diisopropyl-(1-heptenyl)silanol $^{3}(4),(E)-1-[4-(1-h e p t e n y l) p h e n y l]$ ethanone $^{3}(13),(E)-4-m e t h o x y-2-\left(1-\right.$ pentenyl)benzene $^{5}$ (14), (E)-1-(1-heptenyl)-4-methoxybenzene ${ }^{3}$ (15), (E)-1-(1-heptenyl)-2-methylbenzene 3 (17), 4(trimethylsilyl)ethynylbenzaldehyde ${ }^{6}(\mathbf{1 8})$ and $\left(i-\operatorname{Pr}_{3} \mathrm{P}\right)_{2} \operatorname{RuHCl}(\mathrm{CO})^{7}$.

\section{Experimental Procedures}

\section{Preparation of Alkenylsilane Precursors.}

(E)-Dimethyl-(1-pentenyl)silanol (1)

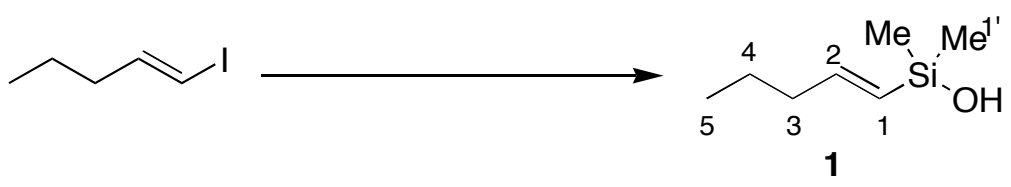

To a solution of $(E)$-1-iodo-1-pentene $(4.90 \mathrm{~g}, 25.0 \mathrm{mmol})$ in diethyl ether $(50 \mathrm{~mL})$ under dry $\mathrm{N}_{2}$ at $-78{ }^{\circ} \mathrm{C}$, was added $n$-butyllithium (16.0 mL, $25.0 \mathrm{mmol}, 1.55 \mathrm{M}, 1.0$ equiv) over 10 min and the reaction mixture was stirred at $-78{ }^{\circ} \mathrm{C}$ for $30 \mathrm{~min}$. A solution of hexamethylcyclotrisiloxane $(1.854 \mathrm{~g}, 8.33 \mathrm{mmol}, 0.33$ equiv) in diethyl ether $(30 \mathrm{~mL})$ was then added over $5 \mathrm{~min}$ at $-78^{\circ} \mathrm{C}$. The mixture was warmed to room temperature and was stirred for $24 \mathrm{~h}$. The solution was then cooled to $0{ }^{\circ} \mathrm{C}$ and was quenched with water $(15 \mathrm{~mL})$. The aqueous phase was extracted with diethyl ether $(3 \times 20 \mathrm{~mL})$ and the combined organic extracts were washed with water $(1 \times 20 \mathrm{~mL})$ and brine $(3 \times 25 \mathrm{~mL})$. The organic layer was dried $\left(\mathrm{MgSO}_{4}\right)$ and filtered. The solvent was then evaporated in vacuo to give an yellow oil which was purified by distillation to afford $3.06 \mathrm{~g}(85 \%)$ of $\mathbf{1}$ as a colorless oil. Repeated distillation provided analytically pure material.

\section{Data for 1:}

bp: $\quad 113{ }^{\circ} \mathrm{C}(100 \mathrm{mmHg})$

${ }^{1} \underline{\mathrm{H} \mathrm{NMR}}: \quad\left(500 \mathrm{MHz}, \mathrm{CDCl}_{3}\right)$

$6.18(\mathrm{dt}, J=18.9,6.2,1 \mathrm{H}, \mathrm{HC}(2)), 5.65$ (dt, $J=18.9,1.5,1 \mathrm{H}, \mathrm{HC}(1)), 2.10$ (qd, $\left.J=7.0,1.7,2 \mathrm{H}, \mathrm{H}_{2} \mathrm{C}(3)\right), 1.58(\mathrm{~s}, \mathrm{OH}, 1 \mathrm{H}), 1.43$ (sext, $\left.J=7.3,2 \mathrm{H}, \mathrm{H}_{2} \mathrm{C}(4)\right)$, 
$0.90\left(\mathrm{t}, J=7.3,3 \mathrm{H}, \mathrm{H}_{3} \mathrm{C}(5)\right), 0.19\left(\mathrm{~s}, 6 \mathrm{H}, 2 \mathrm{H}_{3} \mathrm{C}\left(1^{\prime}\right)\right)$

${ }^{13}$ C NMR: $\quad\left(101 \mathrm{MHz}, \mathrm{CDCl}_{3}\right)$

$149.2(\mathrm{C}(1)), 128.4(\mathrm{C}(2)), 38.6(\mathrm{C}(3)), 21.6(\mathrm{C}(4)), 13.7(\mathrm{C}(5)), 0.0\left(\mathrm{C}\left(1^{\prime}\right)\right)$

IR: $\quad(\mathrm{NaCl})$

3271 (s), 2960 (s), 2931 (s), 2875 (s), 1620 (s), 1252 (s), 991 (s), 866 (s), 843 (s)

MS: $\quad(E I, 70 \mathrm{eV})$

$144\left(\mathrm{M}^{+}, 3.0\right), 129(100), 116(5), 101(9), 75$ (20), 61 (24)

TLC: $\quad R_{f} 0.14$ (pentane/Et $\left.2 \mathrm{O}, 9 / 1\right)\left[\mathrm{KMnO}_{4}\right]$

Analysis: $\quad \mathrm{C}_{7} \mathrm{H}_{16} \mathrm{OSi}(144.29)$

Calc.: $\quad$ C, $58.27 ; \quad H, 11.18 \%$

Found: $\quad$ C, $57.92 ; \quad H, 11.32 \%$

\section{(E)-Diethyl-(1-heptenyl)silanol (3)}

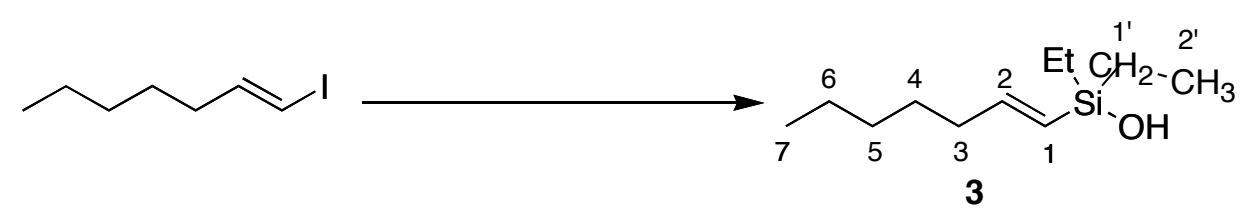

To a solution of $(E)$-1-iodo-1-heptene $(6.72 \mathrm{~g}, 30.0 \mathrm{mmol})$ in diethyl ether $(60 \mathrm{~mL})$ under dry $\mathrm{N}_{2}$ at $-78{ }^{\circ} \mathrm{C}$, was added $n$-butyllithium (19.4 mL, $30.0 \mathrm{mmol}, 1.55 \mathrm{M}, 1.0$ equiv) over 10 min and the reaction mixture was stirred at $-78{ }^{\circ} \mathrm{C}$ for $30 \mathrm{~min}$. A solution of hexaethylcyclotrisiloxane ( $3.07 \mathrm{~g}, 10 \mathrm{mmol}, 0.33$ equiv) in diethyl ether $(15 \mathrm{~mL})$ was then added over $5 \mathrm{~min}$ at $-78{ }^{\circ} \mathrm{C}$. The mixture was warmed to room temperature and was stirred for $24 \mathrm{~h}$. The solution was then cooled to $0{ }^{\circ} \mathrm{C}$ and quenched with water $(30 \mathrm{~mL})$. The aqueous phase was extracted with diethyl ether $(3 \times 25 \mathrm{~mL})$ and the combined organic extracts were washed with water $(1 \times 25 \mathrm{~mL})$ and brine $(2 \times 30 \mathrm{~mL})$. The organic layer was dried $\left(\mathrm{MgSO}_{4}\right)$ and filtered. The solvent was then evaporated in vacuo to give an yellow oil which was purified by distillation to afford $4.97 \mathrm{~g}(83 \%)$ of $\mathbf{3}$ as a colorless oil.

Data for 3:

bp: $\quad 93^{\circ} \mathrm{C}(0.8 \mathrm{mmHg})$

${ }^{1} \underline{\mathrm{H} \mathrm{NMR}}: \quad\left(500 \mathrm{MHz}, \mathrm{CDCl}_{3}\right)$

$6.20(\mathrm{dt}, J=18.8,6.3,1 \mathrm{H}, \mathrm{HC}(2)), 5.59$ (dt, $J=18.8,1.5,1 \mathrm{H}, \mathrm{HC}(1)), 2.13$ (qd, 
$J=7.1,1.5,2 \mathrm{H}, \mathrm{HC}(3)), 1.52$ (brs, $\mathrm{OH}, 1 \mathrm{H}), 1.40$ (qn, $J=7.3,2 \mathrm{H}, \mathrm{H}_{2} \mathrm{C}(4)$ ), $1.29\left(\mathrm{~m}, 4 \mathrm{H}, \mathrm{H}_{2} \mathrm{C}(5)\right.$ and $\left.\mathrm{H}_{2} \mathrm{C}(6)\right), 0.97\left(\mathrm{t}, J=7.8,6 \mathrm{H}, 2 \mathrm{H}_{3} \mathrm{C}\left(2^{\prime}\right)\right), 0.88(\mathrm{t}, J=$ 7.1, $\left.3 \mathrm{H}, \mathrm{H}_{3} \mathrm{C}(7)\right), 0.63$ (q, $J=8.1,4 \mathrm{H}, 2 \mathrm{H}_{2} \mathrm{C}\left(1^{\prime}\right)$ )

${ }^{13} \mathrm{C} \mathrm{NMR}: \quad\left(126 \mathrm{MHz}, \mathrm{CDCl}_{3}\right)$

150.3 (C1), 125.6 (C(2)), 36.7 (C(3)), 31.4 (C(4)), 28.2 (C(5)), 22.5 (C(6)), 14.0 $(\mathrm{C}(7)), 6.5\left(\mathrm{C}\left(2^{\prime}\right)\right), 6.4\left(\mathrm{C}\left(2^{\prime}\right)\right)$

IR: $\quad(\mathrm{NaCl})$

3294 (s, br), 2927 (s), 2956 (s), 2875 (s), 1618 (s), 1618 (s), 1460 (s), 1238 (m), 995 (s), 837 (s)

MS: $\quad(\mathrm{EI}, 70 \mathrm{eV})$

$200\left(\mathrm{M}^{+}, 0.6\right), 171(100), 143(6), 115(3), 95$ (7), 75 (50), 61 (5)

TLC: $\quad R_{f} 0.16($ pentane/Et $2 \mathrm{O}, 9 / 1)\left[\mathrm{KMnO}_{4}\right]$

Analysis: $\quad \mathrm{C}_{11} \mathrm{H}_{24} \mathrm{OSi}(200.39)$

Calc.: $\quad$ C, $65.93 ; \quad \mathrm{H}, 12.07 ; \quad \mathrm{Si}, 14.02 \%$

Found: $\quad \mathrm{C}, 65.63 ; \quad \mathrm{H}, 12.26 ; \quad \mathrm{Si}, 14.24 \%$

\section{(E)-Di-tert-butyl-(1-heptenyl)silanol (5)}
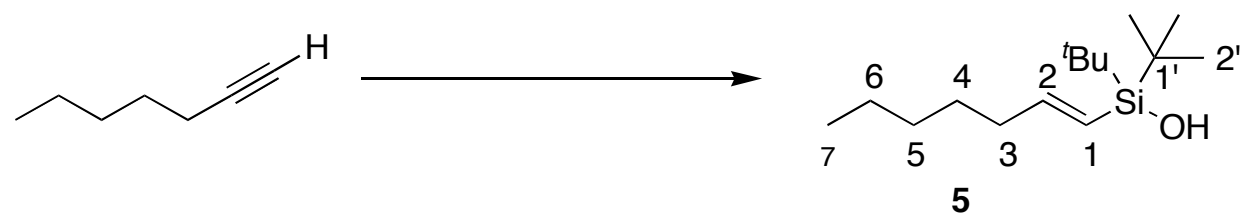

Hexachloroplatinic acid (62 mg, $132 \mu \mathrm{mol}, 0.01$ equiv) was dissolved in $1 \mathrm{~mL}$ of 2 propanol and $10 \mathrm{~mL}$ of diethyl ether in a dry round-bottom flask equipped with a stir bar and a reflux condenser under an atmosphere of dry $\mathrm{N}_{2}$. Chloro(di-tert-butyl)silane (3.4 mL, 16.5 mmol, 1.1 equiv) was then added and the mixture was heated to reflux for $1 \mathrm{~h}$. A solution of 1heptyne $(1.44 \mathrm{~g}, 15 \mathrm{mmol})$ in $5 \mathrm{~mL}$ of dry ether was then added dropwise over $10 \mathrm{~min}$. After the addition was complete, the mixture was heated in an oil bath to reflux for $24 \mathrm{~h}$. After cooling to room temperature, the solvent was evaporated in vacuo and the residual oil was distilled $\left(105^{\circ} \mathrm{C}\right.$ at $0.8 \mathrm{mmHg})$ to give $3.64 \mathrm{~g}(89 \%)$ of the chlorosilane as a colorless liquid.

The intermediate chlorosilane $(3.64 \mathrm{~g}, 13.3 \mathrm{mmol})$ was dissolved in $50 \mathrm{~mL}$ of $\mathrm{THF}$ and a 
sat. aq. solution of $\mathrm{NaHCO}_{3}(30 \mathrm{~mL})$ was added. The mixture was stirred at $50{ }^{\circ} \mathrm{C}$ overnight. The layers were separated and the aqueous phase was washed with diethyl ether (2 x $20 \mathrm{~mL})$. Combined organic layers were washed with water $(2 \times 20 \mathrm{~mL})$ and brine $(2 \times 20 \mathrm{~mL})$ and dried $\left(\mathrm{MgSO}_{4}\right)$, filtered, and the solvents evaporated in vacuo. The resulting oil was purified by column chromatography $\left(\mathrm{SiO}_{2}\right.$, hexane/EtOAc, 9/1) and distilled to give $2.57 \mathrm{~g}(67 \%)$ of 5 as a colorless oil.

Data for 5:

bp: $\quad 117^{\circ} \mathrm{C}(0.8 \mathrm{mmHg})$

${ }^{1} \underline{\mathrm{H} \mathrm{NMR}}: \quad\left(500 \mathrm{MHz}, \mathrm{CDCl}_{3}\right)$

$6.22(\mathrm{dt}, J=18.6,6.3,1 \mathrm{H}, \mathrm{HC}(2)), 5.63$ (dt, $J=18.8,1.5,1 \mathrm{H}, \mathrm{HC}(1)), 2.18$ (qd, $\left.J=7.1,1.2,2 \mathrm{H}, \mathrm{H}_{2} \mathrm{C}(3)\right), 1.44$ (qn, $\left.J=7.3,2 \mathrm{H}, \mathrm{H}_{2} \mathrm{C}(4)\right), 1.40$ (s, OH, $1 \mathrm{H}$ ), 1.32 $\left(\mathrm{m}, 4 \mathrm{H}, \mathrm{H}_{2} \mathrm{C}(5)\right.$ and $\left.\mathrm{H}_{2} \mathrm{C}(6)\right), 1.02\left(\mathrm{~s}, 18 \mathrm{H}, 6 \mathrm{H}_{3} \mathrm{C}\left(2^{\prime}\right)\right), 0.91(\mathrm{t}, J=6.6,3 \mathrm{H}$, $\left.\mathrm{H}_{3} \mathrm{C}(7)\right)$

${ }^{13} \underline{\mathrm{C} \mathrm{NMR}}: \quad\left(126 \mathrm{MHz}, \mathrm{CDCl}_{3}\right)$

$150.1(\mathrm{C}(1)), 126.7(\mathrm{C}(2)), 36.8(\mathrm{C}(3)), 31.3(\mathrm{C}(4)), 28.4(\mathrm{C}(5)), 27.6\left(\mathrm{C}\left(2^{\prime}\right)\right)$, $22.5(\mathrm{C}(6)), 19.8\left(\mathrm{C}\left(1^{\prime}\right)\right), 14.0(\mathrm{C}(7))$

IR: $\quad(\mathrm{NaCl})$ 3469 (s, br), 2966 (s), 2856 (s), 1616 (s), 1470 (s), 1363 (s), 823 (s)

MS: $\quad(\mathrm{EI}, 70 \mathrm{eV})$

$256\left(\mathrm{M}^{+}, 1.0\right), 199$ (83), 181 (20), 157 (26), 129 (99), 115 (67), 75 (100), 61 (37)

TLC: $\quad R_{f} 0.32$ (pentane/Et $\left.2 \mathrm{O}, 9 / 1\right)\left[\mathrm{KMnO}_{4}\right]$

Analysis: $\quad \mathrm{C}_{15} \mathrm{H}_{32} \mathrm{OSi}(256.50)$

Calc.: $\quad$ C, $70.24 ; \quad H, 12.58 \%$

Found: $\quad$ C, $70.11 ; \quad H, 12.88 \%$ 


\section{(E)-Diphenyl-(1-heptenyl)silanol (6)}

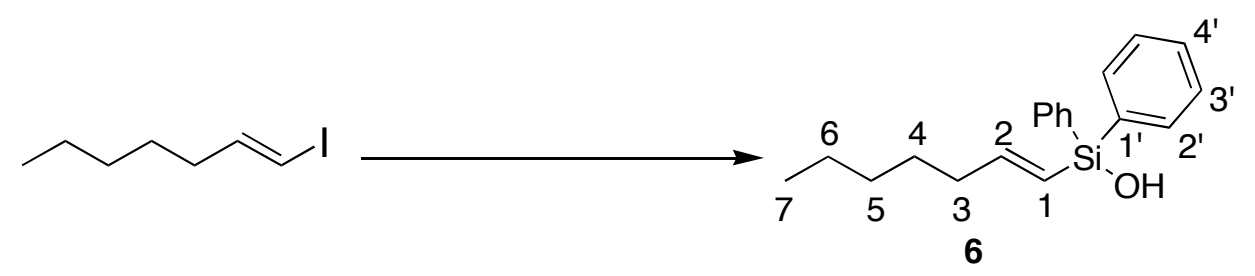

To a solution of $(E)$-1-iodo-1-heptene $(2.29 \mathrm{~g}, 10.2 \mathrm{mmol})$ in diethyl ether $(25 \mathrm{~mL})$ under dry $\mathrm{N}_{2}$ at $-78^{\circ} \mathrm{C}$, was added $n$-butyllithium (6.6 mL, $10.2 \mathrm{mmol}, 1.55 \mathrm{M}, 1.0$ equiv) over $10 \mathrm{~min}$ and the reaction mixture was stirred at $-78{ }^{\circ} \mathrm{C}$ for $30 \mathrm{~min}$. A solution of diphenylchlorosilane (2.0 mL, $10.2 \mathrm{mmol}, 1.0$ equiv) in diethyl ether $(10 \mathrm{~mL})$ was then added over $5 \mathrm{~min}$ at $-78{ }^{\circ} \mathrm{C}$. After stirring for $30 \mathrm{~min}$ at $-78^{\circ} \mathrm{C}$, the mixture was warmed to room temperature and was stirred for $2 \mathrm{~h}$, during which time a white precipitate formed. The solution was then cooled to $0{ }^{\circ} \mathrm{C}$ and was quenched (ice/sat. aq. ammonium chloride, 1/1, $40 \mathrm{~mL}$ ). The aqueous phase was extracted with pentane $(2 \times 20 \mathrm{~mL})$ and the combined organic extracts were washed with brine $(2 \times 20$ $\mathrm{mL})$. The organic layer was dried $\left(\mathrm{MgSO}_{4}\right)$ and filtered. The solvent was then evaporated in vacuo to give an yellow oil which was distilled $\left(146^{\circ} \mathrm{C}\right.$ at $\left.0.8 \mathrm{mmHg}\right)$ to afford $2.28 \mathrm{~g}(79 \%)$ of the hydrosilane.

The intermediate silane $(1.73 \mathrm{~g}, 6.16 \mathrm{mmol})$ was dissolved in $10 \mathrm{~mL}$ of diethyl ether and $\mathrm{Bu}_{4} \mathrm{~N}^{+} \mathrm{OH}^{-}$in $\mathrm{MeOH}$ was slowly dropwise over $5 \mathrm{~min}$, with vigorous gas evolution. After being stirred for $10 \mathrm{~min}$ the mixture was quenched in a mixture of ether and water $(75 \mathrm{~mL}, 30 \mathrm{~mL})$. The phases were separated and the aqueous layer was extracted with ether $(2 \times 25 \mathrm{~mL})$. The combined organic extracts were washed with water $(2 \times 15 \mathrm{~mL})$ and brine $(2 \times 15 \mathrm{~mL})$ then were dried $\left(\mathrm{MgSO}_{4}\right)$, filtered, and the solvent was evaporated in vacuo. The resulting oil was purified by distillation to give $1.66 \mathrm{~g}(92 \%)$ of $\mathbf{6}$ as colorless oil.

Data for 6:

bp: $\quad 165^{\circ} \mathrm{C}(0.8 \mathrm{mmHg})$

${ }^{1}$ H NMR: $\quad\left(500 \mathrm{MHz}, \mathrm{CDCl}_{3}\right)$

$7.65\left(\mathrm{dd}, J=7.7,1.3,4 \mathrm{H}, \mathrm{HC}\left(2^{\prime}\right)\right), 7.39\left(\mathrm{~m}, 6 \mathrm{H}, \mathrm{HC}\left(3^{\prime}\right)\right.$ and $\left.\mathrm{HC}\left(4^{\prime}\right)\right), 6.33(\mathrm{dt}, J$ = 18.7, 2.9, $1 \mathrm{H}, \mathrm{HC}(2)), 5.99$ (d, $J=18.7,1 \mathrm{H}, \mathrm{HC}(1)), 2.22$ (s, OH, $1 \mathrm{H}), 2.21$ (qd, $\left.J=7.7,1.5,2 \mathrm{H}, \mathrm{H}_{2} \mathrm{C}(3)\right), 1.44$ (qn, $\left.J=7.3,2 \mathrm{H}, \mathrm{H}_{2} \mathrm{C}(4)\right), 1.31$ (m, $4 \mathrm{H}$, $\mathrm{H}_{2} \mathrm{C}(5)$ and $\left.\mathrm{H}_{2} \mathrm{C}(6)\right), 0.89$ (t, $J=6.9,3 \mathrm{H}, \mathrm{H}_{3} \mathrm{C}(7)$ ) 
${ }^{13}$ C NMR: $\quad\left(126 \mathrm{MHz}, \mathrm{CDCl}_{3}\right)$

$153.7(\mathrm{C} 1), 136.0\left(\mathrm{C}\left(1^{\prime}\right)\right), 134.6\left(\mathrm{C}\left(2^{\prime}\right)\right), 129.9\left(\mathrm{C}\left(4^{\prime}\right)\right), 127.8\left(\mathrm{C}\left(3^{\prime}\right)\right), 124.0(\mathrm{C}(2))$, $36.7(\mathrm{C}(3)), 31.4(\mathrm{C}(4)), 28.0(\mathrm{C}(5)), 22.5(\mathrm{C}(6)), 14.0(\mathrm{C}(7))$

IR: $\quad(\mathrm{NaCl})$

3278 (s, br), 3068 (s), 3049 (s), 2956 (s), 2927 (s), 2856 (s), 1616 (s), 1429 (s), 1118 (s), 997 (s), 849 (s), 878 (s)

MS: (EI, $70 \mathrm{eV})$ 296 (M+1 16), 239 (17), 225 (65), 199 (100), 123 (59), 77 (17), 58 (9)

TLC: $\quad R_{f} 0.21$ (pentane/Et $\left.2 \mathrm{O}, 9 / 1\right)\left[\mathrm{KMnO}_{4}+\mathrm{UV}\right]$

CG: $\quad t_{\mathrm{R}} 4.93 \mathrm{~min}(>99 \%)\left(\mathrm{HP} 5\right.$, injector $225^{\circ} \mathrm{C}$, column $\left.200{ }^{\circ} \mathrm{C}, 15 \mathrm{psi}\right)$

Analysis: $\quad \mathrm{C}_{19} \mathrm{H}_{20} \mathrm{O}_{1} \mathrm{Si}(172.34)$

Calc.: $\quad$ C, $76.97 ; \quad \mathrm{H}, 8.16 ; \quad \mathrm{Si}, 9.47 \%$

Found $\quad \mathrm{C}, 76.75 ; \quad \mathrm{H}, 8.16 ; \quad \mathrm{Si}, 9.67 \%$

\section{(E)-(1-Heptenyl)triethoxysilane (11)}
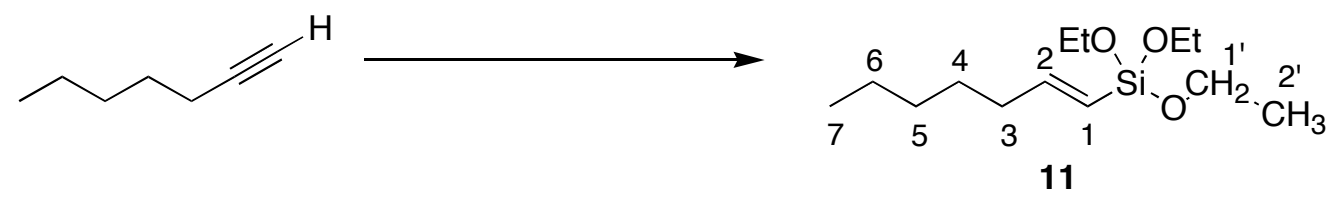

Triethoxysilane (970 $\mu \mathrm{L} 5.3 \mathrm{mmol}, 1.05$ equiv) was combined with a solution of platinum(0)-DVDS- $t$-Bu3 $\mathrm{P}(50 \mu \mathrm{L})$ in xylene. The solution was cooled to $0{ }^{\circ} \mathrm{C}$ and 1-heptyne (655 $\mu \mathrm{L}, 5.0 \mathrm{mmol}$ ) was added. The ice bath was then removed and the reaction was stirred for $4 \mathrm{~h}$. All volatile materials were then removed by evaporation under high vacuum and the residual oil was Kugelrohr distilled at $150{ }^{\circ} \mathrm{C}(3 \mathrm{mmHg})$. The distillate was purified by radial chromatography ( $\mathrm{SiO}_{2}$, pentane/Et $\left.2 \mathrm{O}, 19 / 1\right)$ and then was distilled on a Kugelrohr to give 690 $\mathrm{mg}(52 \%)$ of $\mathbf{1 1}$ as a colorless oil.

\section{Data for 11:}

bp: $\quad 125^{\circ} \mathrm{C}(0.4 \mathrm{mmHg}, \mathrm{ABT})$

${ }^{1}$ H NMR: $\quad\left(500 \mathrm{MHz}, \mathrm{CDCl}_{3}\right)$

$6.42(\mathrm{dt}, J=17.9,6.3,1 \mathrm{H}, \mathrm{HC}(2)), 5.40(\mathrm{dt}, J=18.2,1.6,1 \mathrm{H}, \mathrm{HC}(1)), 3.81$ (q, $J$ $=7.1,6 \mathrm{H}, \mathrm{HC}\left(1^{\prime}\right), 2.13$ (qd, $J=7.4,1.5,2 \mathrm{H}, \mathrm{H}_{2} \mathrm{C}(3)$ ), 1.41 (qn, $J=7.3,2 \mathrm{H}$, 
$\left.\mathrm{H}_{2} \mathrm{C}(4)\right), 1.29\left(\mathrm{~m}, 4 \mathrm{H}, \mathrm{H}_{2} \mathrm{C}(5)\right.$ and $\left.\mathrm{H}_{2} \mathrm{C}(6)\right), 1.22\left(\mathrm{t}, J=7.1,9 \mathrm{H}, 3 \mathrm{H}_{3} \mathrm{C}\left(2^{\prime}\right)\right), 0.88$ $\left(\mathrm{t}, J=6.6,3 \mathrm{H}, \mathrm{H}_{3} \mathrm{C}(7)\right)$

${ }^{13}$ C NMR: $\quad\left(126 \mathrm{MHz}, \mathrm{CDCl}_{3}\right)$

$154.3(\mathrm{C}(1)), 118.9(\mathrm{C}(2)), 58.6\left(\mathrm{C}\left(1^{\prime}\right), 36.7(\mathrm{C}(3)), 31.5(\mathrm{C}(4)), 28.1(\mathrm{C}(5)), 22.6\right.$ $(\mathrm{C}(6)), 18.4\left(\mathrm{C}\left(2^{\prime}\right)\right), 14.2(\mathrm{C}(7))$

IR: $\quad(\mathrm{NaCl})$ 2973 (s), 2927 (s), 1619 (m), 1442 (w), 1390 (m), 1166 (s), 1105 (s), 958 (s)

MS: $\quad(\mathrm{EI}, 70 \mathrm{eV})$

$260\left(\mathrm{M}^{+}, 2\right), 245$ (5), 215 (16), 189 (9), 163 (100), 135 (10), 119 (25)

TLC: $\quad R_{f} 0.23$ (pentane/Et $\left.{ }_{2} \mathrm{O}, 19 / 1\right)\left[\mathrm{KMnO}_{4}\right]$

CG: $\quad t_{\mathrm{R}} 7.74 \mathrm{~min}(>99 \%)\left(\mathrm{HP} 5\right.$, injector $225^{\circ} \mathrm{C}$, column $\left.180{ }^{\circ} \mathrm{C}, 15 \mathrm{psi}\right)$

Analysis: $\quad \mathrm{C}_{13} \mathrm{H}_{28} \mathrm{O}_{3} \mathrm{Si}(260.18)$

Calc.:

C, 59.96;

$\mathrm{H}, 10.85 \%$

Found

C, 59.87;

$\mathrm{H}, 11.13 \%$

\section{(E)-Diethoxy-(1-heptenyl)methylsilane (10)}

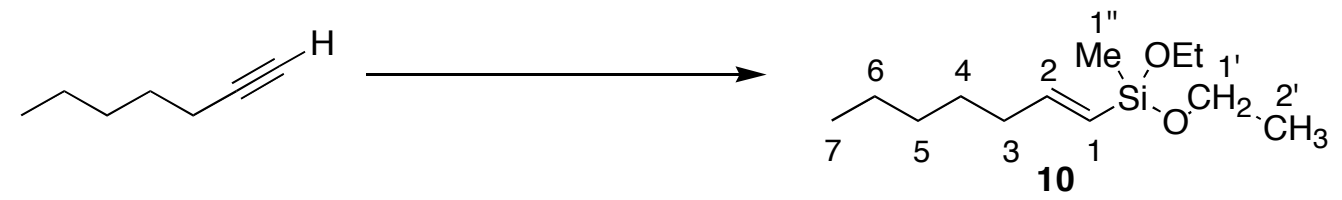

Diethoxymethylsilane ( $850 \mu \mathrm{L} .5 .3 \mathrm{mmol}, 1.05$ equiv) was combined with a solution of platinum(0)-DVDS- $t$ - $\mathrm{Bu}_{3} \mathrm{P}(50 \mu \mathrm{L})$ in xylene. The reaction was cooled to $0{ }^{\circ} \mathrm{C}$ and 1 -heptyne $(655 \mu \mathrm{L}, 5.0 \mathrm{mmol})$ was added. The ice bath was then removed and the reaction was stirred for $4 \mathrm{~h}$. All volatile materials were then removed by evaporation under high vacuo and the residual oil was distilled. The resulting oil was purified by radial chromatography $\left(\mathrm{SiO}_{2}\right.$, pentane/Et $2 \mathrm{O}$, 19/1) and then was distilled to give $563 \mathrm{mg}$ (49\%) of $\mathbf{1 0}$ as a colorless oil.

Data for 10:

bp: $\quad 115^{\circ} \mathrm{C}(0.4 \mathrm{mmHg}, \mathrm{ABT})$

${ }^{1} \underline{\mathrm{H} \mathrm{NMR}}: \quad\left(500 \mathrm{MHz}, \mathrm{CDCl}_{3}\right)$

$6.29(\mathrm{dt}, J=18.5,6.2,1 \mathrm{H}, \mathrm{HC}(2)), 5.52(\mathrm{dt}, J=18.5,1.7,1 \mathrm{H}, \mathrm{HC}(1)), 3.76(\mathrm{q}, J$ $=7.1,4 \mathrm{H}, 2 \mathrm{H}_{2} \mathrm{C}\left(1^{\prime}\right), 2.12\left(\mathrm{qd}, J=7.5,1.5,2 \mathrm{H}, \mathrm{H}_{2} \mathrm{C}(3)\right), 1.39(\mathrm{qn}, J=7.2,2 \mathrm{H}$, 
$\left.\mathrm{H}_{2} \mathrm{C}(4)\right), 1.27$ (m, $4 \mathrm{H}, \mathrm{H}_{2} \mathrm{C}(5)$ and $\left.\mathrm{H}_{2} \mathrm{C}(6)\right), 1.19\left(\mathrm{t}, J=7.1,6 \mathrm{H}, 3 \mathrm{H}_{2} \mathrm{C}\left(2^{\prime}\right)\right), 0.87$ (t, $\left.J=7.0,3 \mathrm{H}, \mathrm{H}_{3} \mathrm{C}(7)\right), 0.16$ (s, $\left.3 \mathrm{H}, \mathrm{H}_{3} \mathrm{C}\left(1^{\prime \prime}\right)\right)$

${ }^{13} \underline{\mathrm{C} \mathrm{NMR}}: \quad\left(126 \mathrm{MHz}, \mathrm{CDCl}_{3}\right)$

$152.3(\mathrm{C}(1)), 123.5(\mathrm{C}(2)), 58.3\left(\mathrm{C}\left(1^{\prime}\right), 36.7\right.$ (C(3)), $31.5(\mathrm{C}(4)), 28.2(\mathrm{C}(5)), 22.7$ $(\mathrm{C}(6)), 18.5\left(\mathrm{C}\left(2^{\prime}\right)\right), 14.2(\mathrm{C}(7)),-4.2\left(\mathrm{C}\left(1^{\prime \prime}\right)\right)$

IR: $\quad(\mathrm{NaCl})$

2970 (s), 2927 (s), 2875 (s), 1620 (m), 1458 (w), 1390 (m), 1255 (m), 1105 (s), 1079 (s), 952 (s), 823 (s)

MS: $\quad(\mathrm{EI}, 70 \mathrm{eV})$

$230\left(\mathrm{M}^{+}, 1\right), 215$ (100), 185 (9), 171 (19), 133 (54), 89 (12), 77 (13)

TLC: $\quad R_{f} 0.22\left(\right.$ pentane $\left./ \mathrm{Et}_{2} \mathrm{O}, 19 / 1\right)\left[\mathrm{KMnO}_{4}\right]$

CG: $\quad t_{\mathrm{R}} 5.35 \mathrm{~min}(>99 \%)\left(\mathrm{HP} 5\right.$, injector $225^{\circ} \mathrm{C}$, column $\left.180{ }^{\circ} \mathrm{C}, 15 \mathrm{psi}\right)$

Analysis: $\quad \mathrm{C}_{12} \mathrm{H}_{26} \mathrm{O}_{2} \mathrm{Si}(230.17)$

Calc.: $\quad$ C, $62.56 ; \quad H, 11.38 \%$

Found: $\quad$ C, $62.37 ; \quad H, 11.33 \%$

\section{(E)-Dimethylethoxy-(1-heptenyl)silane (9)}

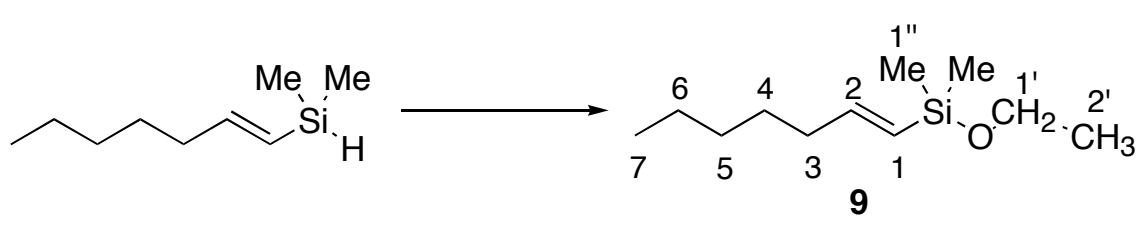

Sodium (16 mg, $0.7 \mathrm{mmol}, 0.01$ equiv) was added to $10 \mathrm{~mL}$ of dry ethanol and the resulting solution was stirred for $30 \mathrm{~min}$. The solution was cooled to $-4{ }^{\circ} \mathrm{C}$ (internal temperature) and the silane $(1.09 \mathrm{~g}, 7.0 \mathrm{mmol})$ was slowly added whereupon gas evolution was observed. After being stirred for $1 \mathrm{~h}$ at room temperature, the mixture was diluted with ether $(50 \mathrm{~mL})$ and then was filtered through a plug of Celite. The solvent was evaporated in vacuo and the resulting oil was distilled to give $952 \mathrm{mg}(68 \%)$ of 9 as colorless oil.

Data for 9:

bp: $\quad 120{ }^{\circ} \mathrm{C}(0.6 \mathrm{mmHg}, \mathrm{ABT})$

${ }^{1} \underline{\mathrm{H} \mathrm{NMR}}: \quad\left(400 \mathrm{MHz}, \mathrm{CDCl}_{3}\right)$

$6.17(\mathrm{dt}, J=18.5,6.1,1 \mathrm{H}, \mathrm{HC}(2)), 5.61$ (dt, $J=18.5,1.7,1 \mathrm{H}, \mathrm{HC}(1)), 3.65$ (q, $J$ 
$=7.1,2 \mathrm{H}, \mathrm{H}_{2} \mathrm{C}\left(1^{\prime}\right), 2.12\left(\mathrm{qd}, J=7.5,1.5,2 \mathrm{H}, \mathrm{H}_{2} \mathrm{C}(3)\right), 1.40$ (qn, $J=7.5,2 \mathrm{H}$, $\mathrm{HC}(4)), 1.29\left(\mathrm{~m}, 4 \mathrm{H}, \mathrm{H}_{2} \mathrm{C}(5)\right.$ and $\left.\mathrm{H}_{2} \mathrm{C}(6)\right), 1.18\left(\mathrm{t}, J=7.1,3 \mathrm{H}, \mathrm{H}_{3} \mathrm{C}\left(2^{\prime}\right)\right), 0.88$ (t, $\left.J=7.0,3 \mathrm{H}, \mathrm{H}_{3} \mathrm{C}(7)\right), 0.16\left(\mathrm{~s}, 3 \mathrm{H}, \mathrm{H}_{3} \mathrm{C}\left(1^{\prime \prime}\right)\right)$

${ }^{13}$ C NMR: $\quad\left(101 \mathrm{MHz}, \mathrm{CDCl}_{3}\right)$

$150.1(\mathrm{C}(1)), 127.2(\mathrm{C}(2)), 58.5\left(\mathrm{C}\left(1^{\prime}\right), 36.8(\mathrm{C}(3)), 31.6(\mathrm{C}(4)), 28.4(\mathrm{C}(5)), 22.7\right.$ $(\mathrm{C}(6)), 18.7\left(\mathrm{C}\left(2^{\prime}\right)\right), 14.2(\mathrm{C}(7)),-1.5\left(\mathrm{C}\left(1^{\prime \prime}\right)\right)$

IR: $\quad(\mathrm{NaCl})$

2969 (s), 2927 (s), 2873 (s), 1618 (m), 1460 (w), 1390 (w), 1250 (s),1109 (s), 1080 (s), 993 (s), 837 (s)

MS: $\quad(E I, 70 \mathrm{eV})$

$200\left(\mathrm{M}^{+},>1 \%\right), 185$ (100), 141 (10), 103 (26)

TLC: $\quad R_{f} 0.30$ (hexane/EtOAc, 20/1) $\left[\mathrm{KMnO}_{4}\right]$

CG: $\quad t_{\mathrm{R}} 5.04 \min (>99 \%)\left(\mathrm{HP} 5\right.$, injector $225^{\circ} \mathrm{C}$, column $\left.170{ }^{\circ} \mathrm{C}, 15 \mathrm{psi}\right)$

HRMS: $\quad$ calcd for $\mathrm{C}_{11} \mathrm{H}_{24} \mathrm{O}_{1} \mathrm{Si}: 200.1596$; found: 200.1601

\section{(E)-Diethoxy-(1-pentenyl)methylsilane (8)}
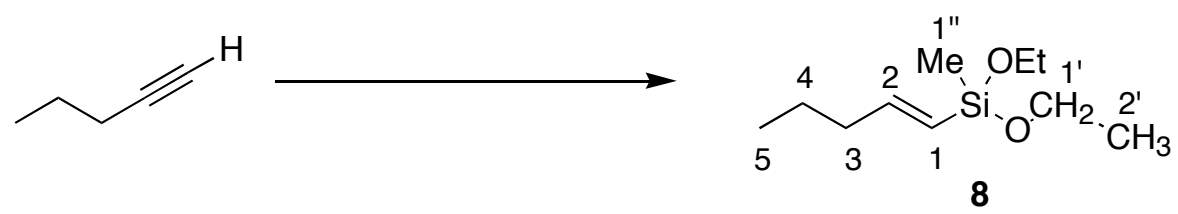

Diethoxymethylsilane ( $850 \mu \mathrm{L} .5 .3 \mathrm{mmol}, 1.05$ equiv) was combined with a solution of platinum(0)-DVDS- $t$ - $\mathrm{Bu}_{3} \mathrm{P}(50 \mu \mathrm{L})$ in xylene. The solution was cooled to $0{ }^{\circ} \mathrm{C}$ and 1 -pentyne (492 $\mu$ L. $5.0 \mathrm{mmol}$ ) was added. The ice bath was then removed and the reaction was stirred for $4 \mathrm{~h}$. All volatile materials were then removed by evaporation under high vacuo and the residual oil was distilled. The resulting oil was purified by radial chromatography $\left(\mathrm{SiO}_{2}\right.$, pentane/Et $2 \mathrm{O}$, 19/1) and distilled to give $450 \mathrm{mg}(43 \%)$ of $\mathbf{8}$ as colorless oil.

Data for 8:

bp: $\quad 95^{\circ} \mathrm{C}(0.8 \mathrm{mmHg}, \mathrm{ABT})$

${ }^{1} \underline{\mathrm{H} \mathrm{NMR}}: \quad\left(400 \mathrm{MHz}, \mathrm{CDCl}_{3}\right)$

$6.28(\mathrm{dt}, J=18.7,6.4,1 \mathrm{H}, \mathrm{HC}(2)), 5.52(\mathrm{dt}, J=19.0,1.4,1 \mathrm{H}, \mathrm{HC}(1)), 3.76$ (q, $J$ $=7.0,4 \mathrm{H}, 2 \mathrm{H}_{2} \mathrm{C}\left(1^{\prime}\right), 2.11$ (qd, $J=7.6,1.5,2 \mathrm{H}, \mathrm{H}_{2} \mathrm{C}(3)$ ), 1.43 (sext, $J=7.3,2 \mathrm{H}$, 
$\left.\mathrm{H}_{2} \mathrm{C}(4)\right), 1.21\left(\mathrm{t}, J=7.0,6 \mathrm{H}, 2 \mathrm{H}_{3} \mathrm{C}\left(2^{\prime}\right)\right), 0.89\left(\mathrm{t}, J=7.3,3 \mathrm{H}, \mathrm{H}_{3} \mathrm{C}(5)\right), 0.16(\mathrm{~s}, 3$ $\left.\mathrm{H}, \mathrm{H}_{3} \mathrm{C}\left(1^{\prime \prime}\right)\right)$

${ }^{13}$ C NMR: $\quad\left(100 \mathrm{MHz}, \mathrm{CDCl}_{3}\right)$

$151.9(\mathrm{C}(1)), 123.8(\mathrm{C}(2)), 58.4\left(\mathrm{C}\left(1^{\prime}\right), 38.8(\mathrm{C}(3)), 21.7(\mathrm{C}(4)), 18.5\left(\mathrm{C}\left(2^{\prime}\right)\right), 13.8\right.$ $(\mathrm{C}(5)),-4.2\left(\mathrm{C}\left(1^{\prime \prime}\right)\right)$

IR: $\quad(\mathrm{NaCl})$

2969 (m), 2927 (m), 2875 (m), 1620 (w), 1390 (w), 1390 (m), 1255 (w), 1166 (s), 1105 (s), 1078 (s), 951 (s), 806 (s)

MS: $\quad(\mathrm{EI}, 70 \mathrm{eV})$

$202\left(\mathrm{M}^{+}, 2.9\right), 187$ (100), 143 (29.3), 133 (37.0), 89 (11.2), 77 (14.0)

TLC: $\quad R_{f} 0.22\left(\right.$ pentane $\left./ \mathrm{Et}_{2} \mathrm{O}, 19 / 1\right)\left[\mathrm{KMnO}_{4}\right]$

Analysis: $\quad \mathrm{C}_{10} \mathrm{H}_{22} \mathrm{O}_{2} \mathrm{Si}(202.14)$

Calc.: $\quad$ C, $59.34 ; \quad H, 10.97 \%$

Found $\quad$ C, 59.10; H, $11.33 \%$

\section{(E)-Trifluoropropylmethyl-(1-heptenyl)silanol (7)}

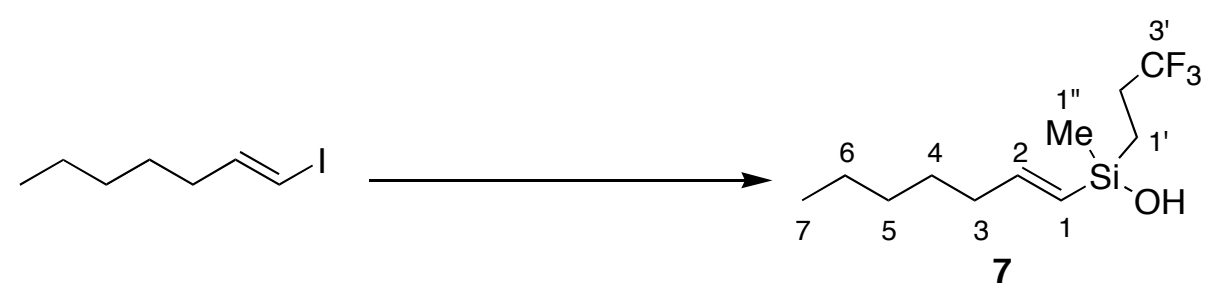

To a solution of $(E)$-1-iodo-1-heptene $(2.0 \mathrm{~g}, 8.9 \mathrm{mmol})$ in dry ether $(15 \mathrm{~mL})$ under dry $\mathrm{N}_{2}$ at $-78{ }^{\circ} \mathrm{C}$ was added a solution of $n$-butyllithium $(5.76 \mathrm{~mL}, 8.9 \mathrm{mmol}, 1.55 \mathrm{M}$ in hexane, 1.0 equiv) dropwise over $10 \mathrm{~min}$. The reaction mixture was stirred at $-78{ }^{\circ} \mathrm{C}$ for $1 \mathrm{~h}$. Then a solution of methyl(1,1,1-trifluoropropyl)cyclotrisiloxane $(1.39 \mathrm{~g}, 2.9 \mathrm{mmol}, 0.33$ equiv) in dry ether $(5 \mathrm{~mL})$ was added over $5 \mathrm{~min}$ at $-78{ }^{\circ} \mathrm{C}$. The mixture was warmed to room temperature and was stirred for $12 \mathrm{~h}$. The solution was then cooled to $0{ }^{\circ} \mathrm{C}$ and was quenched with water (20 $\mathrm{mL})$. The aqueous phase was extracted with ether $(3 \times 25 \mathrm{~mL})$ and the combined organic extracts were washed with water $(1 \times 25 \mathrm{~mL})$ and brine $(2 \times 30 \mathrm{~mL})$. The organic layer was dried with $\mathrm{MgSO}_{4}$ (anhydrous) and was filtered. The solvent was then evaporated in vacuo to give a yellow residue, which was purified by distillation to afford $1.695 \mathrm{~g}(75 \%)$ of 7 as colorless oil. 
Data for 7:

bp: $\quad 105^{\circ} \mathrm{C}(0.4 \mathrm{mmHg}, \mathrm{ABT})$

${ }^{1} \underline{\mathrm{H} \mathrm{NMR}}: \quad\left(500 \mathrm{MHz}, \mathrm{CDCl}_{3}\right)$

$6.22(\mathrm{dt}, J=18.6,6.2,1 \mathrm{H}, \mathrm{HC}(2)), 5.58$ (dt, $J=18.6,1.5,1 \mathrm{H}, \mathrm{HC}(1)), 2.13$ (qd, $\left.J=7.3,1.5,2 \mathrm{H}, \mathrm{H}_{2} \mathrm{C}(3)\right), 2.06\left(\mathrm{~m}, 2 \mathrm{H}, \mathrm{H}_{2} \mathrm{C}\left(2^{\prime}\right)\right) 1.40$ (qn, $\left.J=7.3,2 \mathrm{H}, \mathrm{H}_{2} \mathrm{C}(4)\right)$, 1.29 (m, $4 \mathrm{H}, \mathrm{H}_{2} \mathrm{C}(5)$ and $\left.\mathrm{H}_{2} \mathrm{C}(6)\right), 0.89$ (t, $\left.J=7.1,3 \mathrm{H}, \mathrm{H}_{3} \mathrm{C}(7)\right), 0.85(\mathrm{~m}, 2 \mathrm{H}$, $\left.\mathrm{H}_{2} \mathrm{C}\left(1^{\prime}\right)\right), 0.21$ (s, $\left.3 \mathrm{H}, \mathrm{H}_{3} \mathrm{C}\left(1^{\prime \prime}\right)\right)$

${ }^{13} \mathrm{C} \mathrm{NMR}: \quad\left(126 \mathrm{MHz}, \mathrm{CDCl}_{3}\right)$

$151.6(\mathrm{C}(1)), 128.0$ (q, $J=277,\left(\mathrm{C}\left(3^{\prime}\right)\right), 125.8(\mathrm{C}(2)), 36.8(\mathrm{C}(3)), 31.5(\mathrm{C}(4))$, $28.2\left(\mathrm{q}, J=30 \mathrm{~Hz},\left(\mathrm{C}\left(2^{\prime}\right)\right), 28.2(\mathrm{C}(5)), 22.6(\mathrm{C}(6)), 14.1(\mathrm{C}(7)), 8.7\right.$ (d, $J=1.8$ Hz. $\left.\left(\mathrm{C} 1^{\prime}\right)\right),-1.8\left(\mathrm{C}\left(1^{\prime \prime}\right)\right)$

IR: $\quad(\mathrm{NaCl})$

3269 (s), 2960 (s), 2931 (s), 2859 (s), 1618 (s), 1446 (m), 1365 (m), 1315 (m), 1265 (s), 1209 (s), 1126 (s), 995 (m), 899 (s), 854 (s)

MS: $\quad(\mathrm{EI}, 70 \mathrm{eV})$

157 (M-97, $\left.\mathrm{CH}_{2} \mathrm{CH}_{2} \mathrm{CF}_{3}, 100\right), 95$ (12), 79 (24), 61 (41)

TLC: $\quad R_{f} 0.34$ (hexane/EtOAc, $\left.8 / 1\right)\left[\mathrm{KMnO}_{4}\right]$

CG: $\quad t_{\mathrm{R}} 4.93 \mathrm{~min}(>99 \%)\left(\mathrm{HP} 5\right.$, injector $225^{\circ} \mathrm{C}$, column $\left.200{ }^{\circ} \mathrm{C}, 15 \mathrm{psi}\right)$

Analysis: $\quad \mathrm{C}_{11} \mathrm{H}_{21} \mathrm{O}_{1} \mathrm{~F}_{3} \mathrm{Si}(230.17)$

Calc.: $\quad$ C, $51.94 ; \quad \mathrm{H}, 8.33 \%$

Found $\quad \mathrm{C}, 52.02 ; \quad \mathrm{H}, 8.67 \%$ 


\section{(E)-1-[4-(1-Pentenyl)phenyl]ethanone (12)}

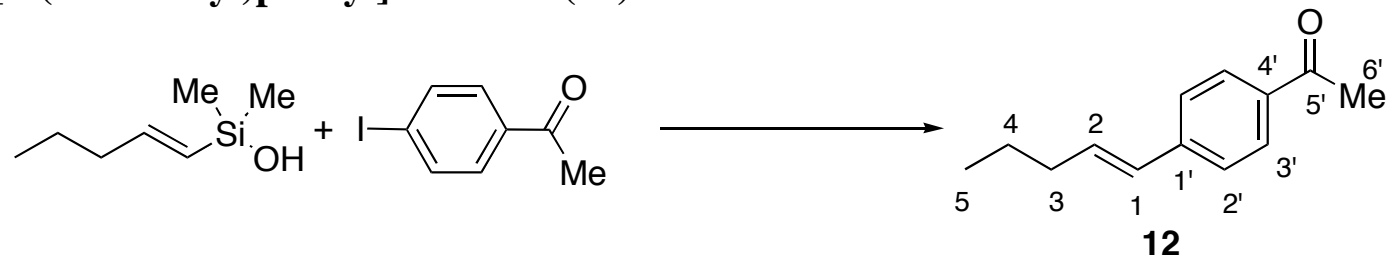

(E)-Dimethyl-(1-pentenyl)silanol ((E)-1 (317 mg, $2.2 \mathrm{mmol}, 1.1$ equiv) was added to a solution of TBAF (4.0 mL, 1.0 M in THF, 2 equiv) and $\mathrm{Pd}(\mathrm{dba})_{2}$ (58 mg. $0.1 \mathrm{mmol}, 0.05$ equiv). 4-Iodoacetophenone (493 mg, $2.0 \mathrm{mmol}$ ) was added and the mixture was stirred for $10 \mathrm{~min}$ at room temperature. The reaction mixture was then filtered through a short silica gel column (20 g). The plug was washed with hexane/EtOAc, 4/1 (200 mL) and the solvent was evaporated in vacuo. The residue was purified by column chromatography (Reverse Phase $\mathrm{C} 18, \mathrm{MeOH} / \mathrm{H}_{2} \mathrm{O}$, 9/1) and distillation to afford $331 \mathrm{mg}(88 \%)$ of $\mathbf{1 2}$ as colorless oil.

Data for 12:

bp: $\quad 130{ }^{\circ} \mathrm{C}(0.8 \mathrm{mmHg}, \mathrm{ABT})$

${ }^{1} \underline{\mathrm{H} \mathrm{NMR}}: \quad\left(500 \mathrm{MHz}, \mathrm{CDCl}_{3}\right)$

$7.89\left(\mathrm{~d}, J=8.5,2 \mathrm{H}, \mathrm{HC}\left(3^{\prime}\right)\right), 7.41$ (d, $\left.J=8.3,2 \mathrm{H}, \mathrm{HC}\left(2^{\prime}\right)\right), 6.39$ (m, $2 \mathrm{H} \mathrm{HC(1)}$ and $\mathrm{HC}(2)), 2.58$ (s, $\left.3 \mathrm{H}, \mathrm{H}_{3} \mathrm{C}\left(6^{\prime}\right)\right), 2.26$ (q, $J=5.9,2 \mathrm{H}, \mathrm{H}_{2} \mathrm{C}(3)$ ), 1.53 (sept, $J=$ $\left.7.3,2 \mathrm{H}, \mathrm{H}_{2} \mathrm{C}(4)\right), 0.97$ (t, $\left.J=7.3,3 \mathrm{H}, \mathrm{H}_{3} \mathrm{C}(5)\right)$

${ }^{13} \underline{\mathrm{C} \mathrm{NMR}}: \quad\left(101 \mathrm{MHz}, \mathrm{CDCl}_{3}\right)$

197.6 (C(5')), $142.6\left(\mathrm{C}\left(4^{\prime}\right)\right), 135.4\left(\mathrm{C}\left(1^{\prime}\right)\right), 134.3$ (C(2)), $129.1(\mathrm{C}(1)), 128.7$ $\left(\mathrm{C}\left(3^{\prime}\right)\right), 125.9\left(\mathrm{C}\left(2^{\prime}\right)\right), 35.2(\mathrm{C}(3)), 26.5\left(\mathrm{C}\left(6^{\prime}\right)\right), 22.3(\mathrm{C}(4)), 13.7(\mathrm{C}(5))$

IR: $\quad(\mathrm{NaCl})$

2960 (s), 2931 (s), 2872 (s), 1680 (s), 1603 (s), 1412 (s), 1358 (s), 1269 (s), 1180 (s), $966(\mathrm{~s}), 850(\mathrm{~s})$

MS: $\quad(\mathrm{EI}, 70 \mathrm{eV})$

$188\left(\mathrm{M}^{+}, 75\right), 173$ (100), 159 (6), 145 (16), 131 (60), 115 (73), 103 (18), 91 (18), 77 (22), 63 (19)

TLC: $\quad R_{f} 0.45$ (hexane/EtOAc, $\left.4 / 1\right)\left[\mathrm{UV}+\mathrm{KMnO}_{4}\right]$

Analysis: $\quad \mathrm{C}_{13} \mathrm{H}_{16} \mathrm{O}(188.27)$

Calc.: $\quad$ C, $82.94 ; \quad H, 8.57 \%$

Found $\quad$ C, 82.65; H, $8.57 \%$ 
(E)-1-Methyl-2-(1-pentenyl)benzene (16)

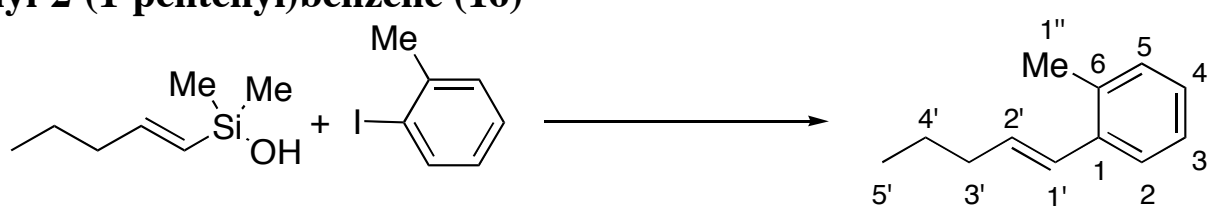

\section{6}

(E)-Dimethyl(1-pentenyl)silanol ((E)-1 (158 mg, $1.1 \mathrm{mmol}, 1.1$ equiv) was added to a solution of TBAF (2.0 mL, $1 \mathrm{M}$ in THF, $2.0 \mathrm{mmol}, 2$ equiv) and $\mathrm{Pd}(\mathrm{dba})_{2}(58 \mathrm{mg} .0 .1 \mathrm{mmol}$, 0.05 equiv). 2-Iodotoluene $(127 \mu \mathrm{L}, 1.0 \mathrm{mmol})$ was added and the mixture was stirred for 30 min at room temperature. The reaction mixture was then filtered through a short silica gel column $(20 \mathrm{~g})$. The plug was washed with hexane/EtOAc, 4/1 (200 mL) and the solvent was evaporated in vacuo. The residue was purified by column chromatography (Reverse Phase C18, $\mathrm{MeOH} / \mathrm{H}_{2} \mathrm{O}$ 9/1) and distillation to afford $128 \mathrm{mg}(80 \%)$ of $\mathbf{1 6}$ as colorless oil.

\section{Data for 16:}

bp: $\quad 100{ }^{\circ} \mathrm{C}(5.0 \mathrm{mmHg}, \mathrm{ABT})$

1․

$7.42(\mathrm{~d}, J=7.3,1 \mathrm{H}, \mathrm{HC}(6)), 7.20$ (m, $3 \mathrm{H} \mathrm{HC(3),} \mathrm{HC(4)} \mathrm{and} \mathrm{HC}(5)), 6.57$ (d, $J=$ 15.6, $\left.1 \mathrm{H}, \mathrm{HC}\left(1^{\prime}\right)\right), 6.09$ (dt, $\left.J=15.6,6.9,1 \mathrm{H}, \mathrm{HC}\left(2^{\prime}\right)\right), 2.33$ (s, $\left.3 \mathrm{H}, \mathrm{H}_{3} \mathrm{C}\left(1^{\prime \prime}\right)\right)$, $2.21\left(\mathrm{qd}, J=7.1,1.2,2 \mathrm{H}, \mathrm{H}_{2} \mathrm{C}\left(3^{\prime}\right)\right), 1.51$ (sept, $\left.J=7.2,2 \mathrm{H}, \mathrm{H}_{2} \mathrm{C}\left(4^{\prime}\right)\right), 0.96$ (t, $J=$ 7.2, $\left.3 \mathrm{H}, \mathrm{H}_{3} \mathrm{C}\left(5^{\prime}\right)\right)$

${ }^{13} \underline{\mathrm{C} \mathrm{NMR}}: \quad\left(126 \mathrm{MHz}, \mathrm{CDCl}_{3}\right)$

137.0 (C(1)), 134.8 (C(2)), $132.3\left(\mathrm{C}\left(1^{\prime}\right)\right), 130.1\left(\mathrm{C}\left(3^{\prime}\right)\right), 127.7\left(\mathrm{C}\left(2^{\prime}\right)\right), 126.6$ (C(4)), 125.9 (C(6)), 125.4 (C(5)), 35.3 (C(3')), 22.5 (C(4')), $16.7\left(\mathrm{C}\left(1^{\prime \prime}\right)\right), 13.6$ $\left(\mathrm{C}\left(5^{\prime}\right)\right)$

IR: $\quad(\mathrm{NaCl})$

3022 (m), 2958 (s), 2927 (s), 1602 (w), 1485 (m), 1461 (m), 1379 (w), 1259 (w), 1045 (m), 964 (s)

MS: $\quad(\mathrm{EI}, 70 \mathrm{eV})$

$160\left(\mathrm{M}^{+}, 44\right), 131$ (100), 115 (21), 91 (31)

TLC: $\quad R_{f} 0.87\left(\mathrm{SiO}_{2}\right.$ hexane $)\left[\mathrm{UV}+\mathrm{KMnO}_{4}\right]$

CG: $\quad t_{\mathrm{R}} 6.32 \min (96 \%)\left(\mathrm{HP} 5\right.$, injector $225^{\circ} \mathrm{C}$, column $\left.200{ }^{\circ} \mathrm{C}, 15 \mathrm{psi}\right)$

HRMS: $\quad$ calcd for $\mathrm{C}_{12} \mathrm{H}_{16}$ : 160.1252 ; found: 160.1254 


\section{(E)-1-[4-(1-Heptenyl)phenyl]ethanone $(13)^{3}$}

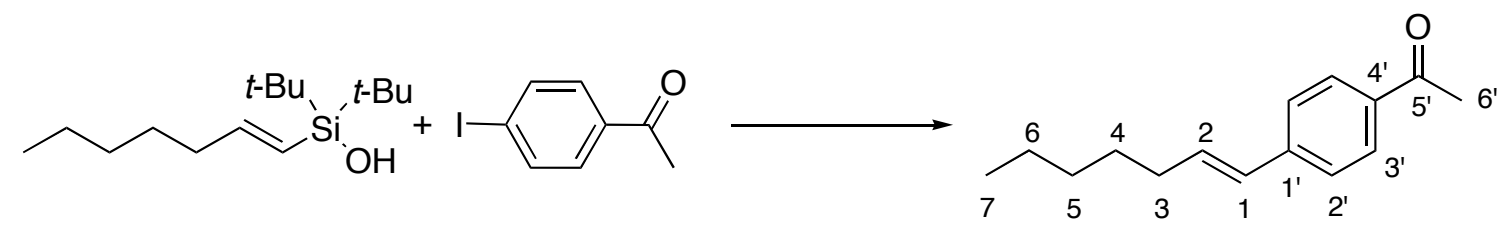

13

(E)-Di-tert-butyl(1-heptenyl)silanol 5 (307 $\mathrm{mg}, 1.2 \mathrm{mmol}, 1.2$ equiv) was added to a solution of TBAF (2.0 mL, $1 \mathrm{M}$ in THF, $2.0 \mathrm{mmol}, 2.0$ equiv) and $\mathrm{Pd}(\mathrm{dba})_{2}(28.7 \mathrm{mg} .0 .05$ mmol, 0.05 equiv). 4-Iodoacetophenone $(246 \mathrm{mg}, 1.0 \mathrm{mmol})$ was added to the mixture. The mixture was stirred at $50{ }^{\circ} \mathrm{C}$ for $19 \mathrm{~h}$. The reaction mixture was then filtered through a short silica gel column $(20 \mathrm{~g})$. The plug was washed with hexane/EtOAc, 4/1 $(50 \mathrm{~mL})$ and the solvent was evaporated in vacuo. The residue was purified by column chromatography $\left(\mathrm{SiO}_{2}\right.$, hexane/EtOAc, 9/1 then hexane/EtOAc, 4/1) to afford product 13 and $29 \mathrm{mg}$ (12\%) of 1,1'-[1,1'biphenyl]-4,4'-diyl-bisethanone (homocoupling product). Further purification by Kugelrohr distillation afforded $331 \mathrm{mg}$ (59\%) of $\mathbf{1 3}$ as colorless oil. ${ }^{3}$

Data for 13:

${ }^{1}$ H NMR: $\quad\left(500 \mathrm{MHz}, \mathrm{CDCl}_{3}\right)$

$7.89\left(\mathrm{~d}, J=8.5,2 \mathrm{H}, \mathrm{HC}\left(3^{\prime}\right)\right), 7.41\left(\mathrm{~d}, J=8.3,2 \mathrm{H}, \mathrm{HC}\left(2^{\prime}\right)\right), 6.39$ (m, $2 \mathrm{H}, \mathrm{HC}(1)$ and $\mathrm{HC}(2)), 2.58$ (s, $\left.3 \mathrm{H}, \mathrm{H}_{3} \mathrm{C}\left(6^{\prime}\right)\right), 2.26$ (q, $J=5.9,2 \mathrm{H}, \mathrm{H}_{2} \mathrm{C}(3)$ ), 1.53 (sept, $J=$ 7.3, $\left.2 \mathrm{H}, \mathrm{H}_{2} \mathrm{C}(4)\right), 0.97$ (t, $\left.J=7.3,3 \mathrm{H}, \mathrm{H}_{3} \mathrm{C}(5)\right)$

${ }^{13}$ C NMR: $\quad\left(101 \mathrm{MHz}, \mathrm{CDCl}_{3}\right)$

$197.6\left(\mathrm{C}\left(5^{\prime}\right)\right), 142.6\left(\mathrm{C}\left(4^{\prime}\right)\right), 135.4\left(\mathrm{C}\left(1^{\prime}\right)\right), 134.3(\mathrm{C}(2)), 129.1(\mathrm{C}(1)), 128.7$ $\left(\mathrm{C}\left(3^{\prime}\right)\right), 125.9\left(\mathrm{C}\left(2^{\prime}\right)\right), 35.2(\mathrm{C}(3)), 26.5\left(\mathrm{C}\left(6^{\prime}\right)\right), 22.3(\mathrm{C}(4)), 13.7(\mathrm{C}(5))$

TLC: $\quad R_{f} 0.45$ (hexane/EtOAc, $4 / 1$ ) [UV $\left.+\mathrm{KMnO}_{4}\right]$

CG: $\quad t_{\mathrm{R}} 8.30 \mathrm{~min}(>99 \%)\left(\mathrm{HP} 5\right.$, injector $225^{\circ} \mathrm{C}$, column $\left.250^{\circ} \mathrm{C}, 15 \mathrm{psi}\right)$ 
Preparation of Ethoxy(di-(1-methylethyl)) 2-[4-[(E)-2-(Ethoxydimethylsilyl)ethenyl]phenyl]ethenylsilane

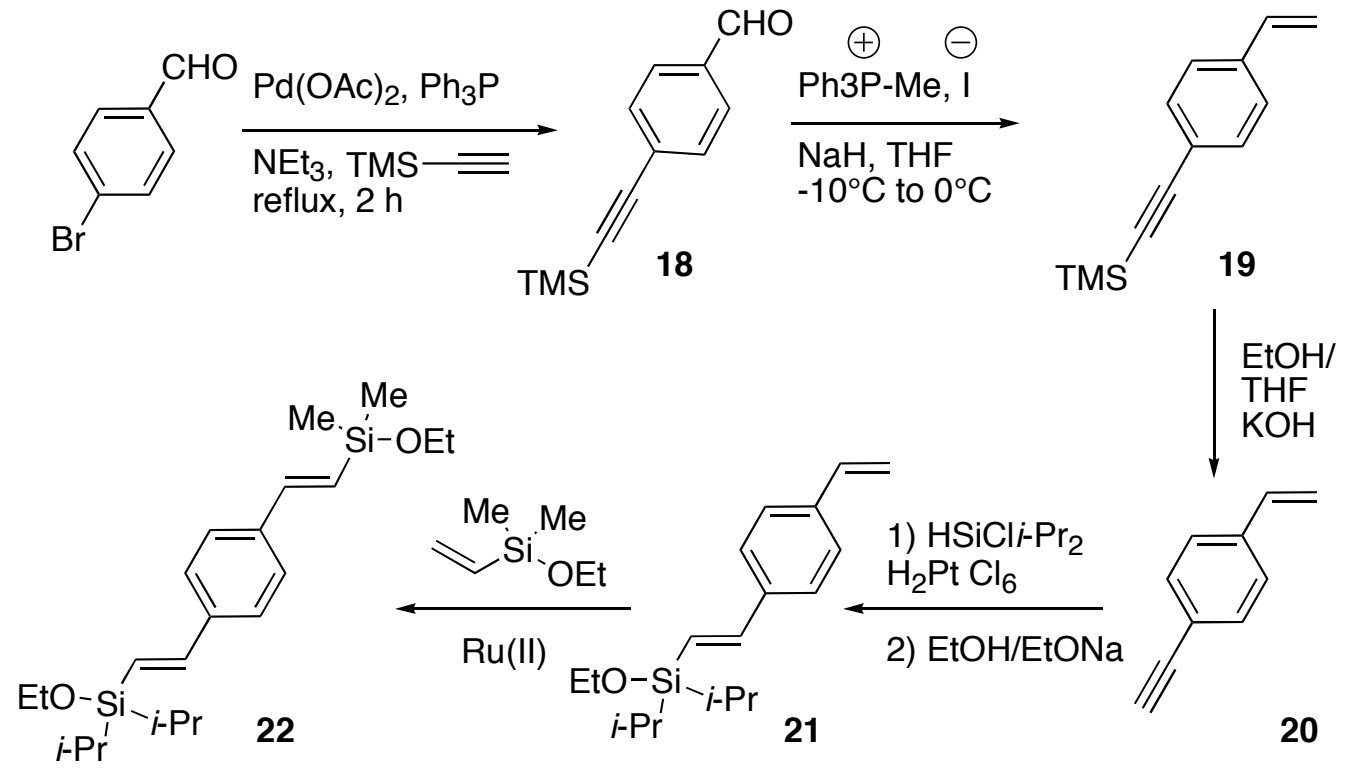

\section{[(4-(Ethenylphenyl)ethynyl] trimethylsilane $(19)^{8}$}

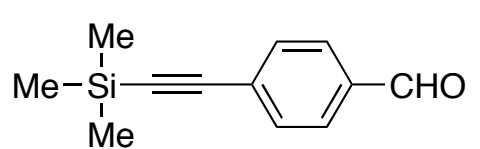

18

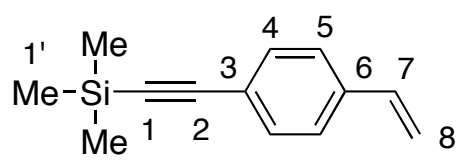

19

To a suspension of triphenylphosphonium iodide ( $4.85 \mathrm{~g}, 12.0 \mathrm{mmol}, 1.1$ equiv) in $10 \mathrm{~mL}$ of THF at $-20{ }^{\circ} \mathrm{C}$, was added a solution of $n$-butyllithium $(7.75 \mathrm{~mL}, 1.55 \mathrm{M}$ in hexane, 12.0 mmol, 1.1 equiv). The reaction was stirred for $30 \mathrm{~min}$ at $-10{ }^{\circ} \mathrm{C}$, then for $1 \mathrm{~h}$ at room temperature. The mixture was cooled to $-20^{\circ} \mathrm{C}$ and a solution of aldehyde $\mathbf{1 8}(2.0 \mathrm{~g}, 10.0 \mathrm{mmol})$ in $5 \mathrm{~mL}$ of THF was slowly added. The reaction was allowed to warm to room temperature and was stirred for $1 \mathrm{~h}$ whereupon the reaction mixture was quenched with water $(30 \mathrm{~mL})$. The aqueous phase was extracted with ether $(3 \times 25 \mathrm{~mL})$ and the combined organic extracts were washed with water $(1 \times 25 \mathrm{~mL})$ and brine $(2 \times 30 \mathrm{~mL})$. The organic layer was dried with $\mathrm{MgSO}_{4}$ (anhydrous) and filtered. After evaporation of the solvent, the residue was purified by column chromatography $\left(\mathrm{SiO}_{2}\right.$, hexane) and distillation to afford $1.60 \mathrm{~g}(81 \%)$ of $\mathbf{1 9}^{8}$ as colorless oil. Data for 19:

mp: $\quad 90^{\circ} \mathrm{C}(0.4 \mathrm{mmHg}, \mathrm{ABT})$ 
${ }^{1} \underline{\mathrm{H} \text { NMR: }} \quad\left(400 \mathrm{MHz}, \mathrm{CDCl}_{3}\right)$

$7.42(\mathrm{~d}, J=8.3,2 \mathrm{H}, \mathrm{HC}(4)), 7.36$ (d, $J=8.1,2 \mathrm{H}, \mathrm{HC}(5)), 6.70(\mathrm{dd}, J=17.1$, 11.0, $1 \mathrm{H}, \mathrm{HC}(7), 5.78$ (d, $J=17.1,1 \mathrm{H}, \mathrm{HC}(8)), 5.31$ (d, $J=11.0,1 \mathrm{H}, \mathrm{HC}(8))$, 3.12 (s, $1 \mathrm{H}, \mathrm{HC}(1))$

${ }^{13} \underline{\mathrm{C} \mathrm{NMR}}: \quad\left(101 \mathrm{MHz}, \mathrm{CDCl}_{3}\right)$

$137.8(\mathrm{C}(6)), 136.4(\mathrm{C}(7)), 132.3(\mathrm{C}(4), 126.2$ (C(5)), $122.6(\mathrm{C}(3)), 115.0(\mathrm{C}(8))$, $105.3(\mathrm{C}(2)), 95.0(\mathrm{C}(1)), 0.2\left(\mathrm{C}\left(1^{\prime}\right)\right)$

TLC: $\quad R_{f} 0.35$ (hexane) $\left[\mathrm{UV}+\mathrm{KMnO}_{4}\right]$

\section{4-Ethenyl-1-ethynylbenzene (20) ${ }^{9}$}

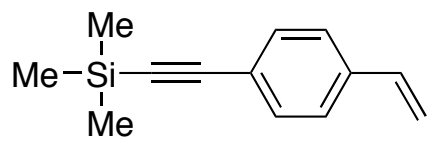

19

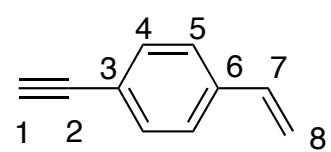

20

To a solution of $19(1.40 \mathrm{~g}, 7.0 \mathrm{mmol})$ in $10 \mathrm{~mL}$ of THF and $10 \mathrm{~mL}$ of ethanol, cooled to $0{ }^{\circ} \mathrm{C}$ (external ice bath) was added an aqueous solution of $\mathrm{KOH}(7.0 \mathrm{~mL}, 7.0 \mathrm{mmol}, 1.0 \mathrm{M}, 1.0$ equiv). The reaction was warmed to room temperature and was stirred for $1 \mathrm{~h}$ whereupon the reaction was quenched with water $(50 \mathrm{~mL})$. The aqueous phase was extracted with ethyl acetate $(3 \times 35 \mathrm{~mL})$ and the combined organic extracts were washed with water $(1 \times 30 \mathrm{~mL})$ and brine $(2$ $\times 30 \mathrm{~mL}$ ). The organic layer was dried with $\mathrm{MgSO}_{4}$ (anhydrous) and filtered. After evaporation of the solvent, the residue was purified by column chromatography $\left(\mathrm{SiO}_{2}\right.$, hexane) and distillation to afford $786 \mathrm{mg}(88 \%)$ of $\mathbf{2 0}$ as colorless oil. ${ }^{9}$

Data for 20:

bp: $\quad 60^{\circ} \mathrm{C}(0.4 \mathrm{mmHg}, \mathrm{ABT})$

${ }^{1} \underline{\mathrm{H} \mathrm{NMR}}: \quad\left(500 \mathrm{MHz}, \mathrm{CDCl}_{3}\right)$

$7.46(\mathrm{~d}, J=8.1,2 \mathrm{H}, \mathrm{HC}(4)), 7.33(\mathrm{~d}, J=8.3,2 \mathrm{H}, \mathrm{HC}(5)), 6.69(\mathrm{dd}, J=17.5$, 11.0, $1 \mathrm{H}, \mathrm{HC}(7), 5.76(\mathrm{~d}, J=17.5,1 \mathrm{H}, \mathrm{HC}(8)), 5.29(\mathrm{~d}, J=11.0,1 \mathrm{H}, \mathrm{HC}(8))$, $0.26\left(\mathrm{~s}, 9 \mathrm{H}, 3 \mathrm{H}_{3} \mathrm{C}\left(1^{\prime}\right)\right)$

${ }^{13}$ C NMR: $\quad\left(126 \mathrm{MHz}, \mathrm{CDCl}_{3}\right)$

138.2 (C(6)), 136.3 (C(7)), 132.5 (C(4), 126.3 (C(5)), $121.5(\mathrm{C}(3)), 115.3(\mathrm{C}(8))$, 83.8(C(2)), 77.9 (C(1)) 
TLC: $\quad R_{f} 0.33$ (hexane) $\left[\mathrm{UV}+\mathrm{KMnO}_{4}\right]$

Ethoxy(di-(1-methylethyl)) 2-[4-[(E)-2-(Ethoxydimethylsilyl)ethenyl]phenyl]ethenylsilane (22)

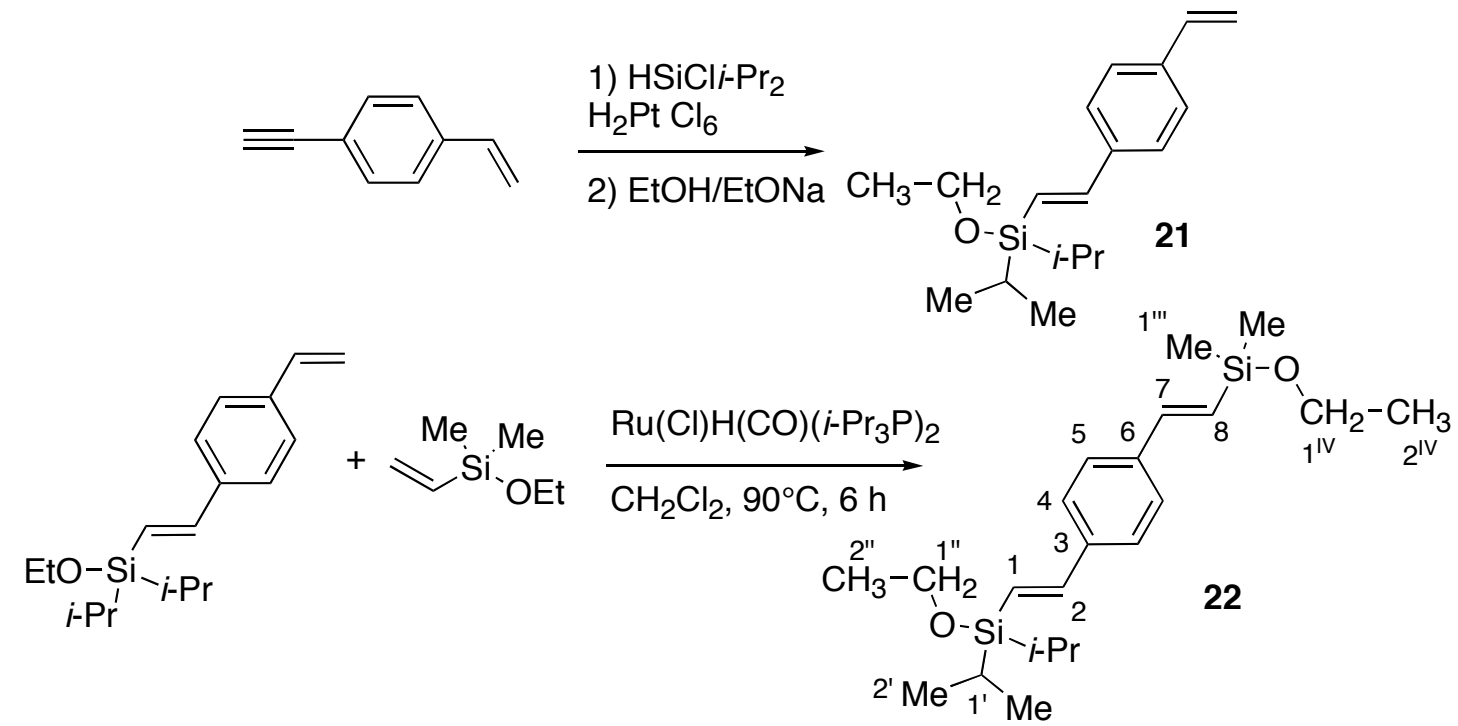

A mixture of $20(740 \mathrm{mg}, 5.7 \mathrm{mmol})$ and diisopropylchlorosilane $(1.05 \mathrm{~mL}, 6.0 \mathrm{mmol}$, 1.05 equiv) and $\operatorname{Pt}(0)$-DVDS solution $(25 \mu \mathrm{L})$. The mixture was stirred for $2.5 \mathrm{~h}$ at room temperature and all volatile materials were removed under high vacuum. The residue was dissolved in $10 \mathrm{~mL}$ of hexane and $1.0 \mathrm{~mL}$ of ethanol (17 mmol, 3.0 equiv) and triethylamine (1.2 $\mathrm{mL}, 8.6 \mathrm{mmol}, 1.5$ equiv) was added. The reaction mixture was further stirred for $1 \mathrm{~h}$ and was filtered. The filtrate was evaporated in vacuo and the resulting oil was purified by column chromatography $\left(\mathrm{SiO}_{2}\right.$, hexane/ $\left.\mathrm{CH}_{2} \mathrm{Cl}_{2}, 7 / 1\right)$ to afford $(E)-21$ as colorless oil.

Compound 21 was placed in a Schlenk tube with $3 \mathrm{~mL}$ of dichloromethane, followed by ethoxydimethylvinylsilane ( $1.03 \mathrm{ml}, 6.3 \mathrm{mmol}, 1.2$ equiv). The tube was placed in a dry-box and Ruthenium catalyst ( $13 \mathrm{mg}, 0.026 \mathrm{mmol}, 0.5 \%$ mol equiv) was added. The tube was sealed, removed from the dry-box and heated at $100{ }^{\circ} \mathrm{C}$ for $12 \mathrm{~h}$. After being cooled to room temperature, the solvent was evaporated. The residue was purified by column chromatography $\left(\mathrm{SiO}_{2}\right.$, hexane/ $\left.\mathrm{CH}_{2} \mathrm{Cl}_{2} 2 / 1\right)$ and distillation to afford $1.29 \mathrm{~g}(58 \%)$ of $\mathbf{2 2}$ as colorless oil.

Data for 22:

bp: $\quad 190{ }^{\circ} \mathrm{C}(0.3 \mathrm{mmHg}, \mathrm{ABT})$ 
${ }^{1} \underline{\mathrm{H} \text { NMR}}: \quad\left(400 \mathrm{MHz}, \mathrm{CDCl}_{3}\right)$

7.44 (s, 4 H, HC(4) and $\mathrm{HC}(5)), 7.03$ (d, $J=19.5,1 \mathrm{H}, \mathrm{HC}(2)), 6.97$ (d, $J=19.3$, $1 \mathrm{H}, \mathrm{HC}(7)), 6.43(\mathrm{~d}, J=19.3,1 \mathrm{H}, \mathrm{HC}(8)), 6.36(\mathrm{~d}, J=19.5,1 \mathrm{H}, \mathrm{HC}(1)), 3.81$ (q, $J=7.1,2 \mathrm{H}, \mathrm{H}_{2} \mathrm{C}\left(1^{\mathrm{IV}}\right), 3.72$ (q, $J=7.1,2 \mathrm{H}, \mathrm{H}_{2} \mathrm{C}\left(1^{\prime \prime}\right), 1.23\left(\mathrm{~m}, 6 \mathrm{H}, \mathrm{H}_{3} \mathrm{C}\left(2^{\prime \prime}\right)\right.$ and $\left.\mathrm{H}_{3} \mathrm{C}\left(2^{\mathrm{IV}}\right)\right), 1.07\left(\mathrm{~m}, 14 \mathrm{H}, 4 \mathrm{H}_{3} \mathrm{C}\left(2^{\prime}\right)\right.$ and $\left.2 \mathrm{HC}\left(1^{\prime}\right)\right), 0.28\left(\mathrm{~s}, 6 \mathrm{H}, 2 \mathrm{H}_{3} \mathrm{C}\left(1^{\prime \prime \prime}\right)\right)$

${ }^{13} \underline{\mathrm{C} \mathrm{NMR}}: \quad\left(101 \mathrm{MHz}, \mathrm{CDCl}_{3}\right)$

$146.3(\mathrm{C}(2)), 145.2$ (C(7)), 138.4(C(3)), 138.0 (C(6)), 127.0 (C(4)), 126.9 (C(5)), $126.9(\mathrm{C}(8)), 122.9(\mathrm{C}(1)), 59.5\left(\mathrm{C}\left(1^{\mathrm{IV}}\right)\right), 58.7\left(\mathrm{C}\left(1^{\mathrm{N}}\right)\right), 18.9\left(\mathrm{C}\left(2^{\mathrm{IV}}\right)\right), 18.7$ $\left(\mathrm{C}\left(2^{\prime \prime}\right)\right), 17.7 / 17.6\left(\mathrm{C}\left(2^{\prime}\right)\right), 12.7(\mathrm{C}(1)),-1.5\left(\mathrm{C}\left(1^{\prime \prime \prime}\right)\right)$

IR: $\quad(\mathrm{NaCl})$

2960 (s), 2865 (s), 1605 (m), 1556 (w), 1506 (w), 1463 (m), 1390 (m), 1251 (s), 1081 (s), 989 (s), 843 (s)

MS: $\quad(E I, 70 \mathrm{eV})$

$390\left(\mathrm{M}^{+}, 6\right), 375(2), 347$ (100), 103 (32), 75 (10)

TLC: $\quad R_{f} 0.26$ (hexane $\left./ \mathrm{CH}_{2} \mathrm{Cl}_{2}, 2 / 1\right)\left[\mathrm{UV}+\mathrm{KMnO}_{4}\right]$

CG: $\quad t_{\mathrm{R}} 16.3 \min (>99 \%)\left(\mathrm{HP} 5\right.$, injector $225^{\circ} \mathrm{C}$, column $\left.250^{\circ} \mathrm{C}, 15 \mathrm{psi}\right)$

HRMS: calc for $\mathrm{C}_{22} \mathrm{H}_{38} \mathrm{O}_{2} \mathrm{Si}_{2}$ : 390.2410 ; found: 390.2410

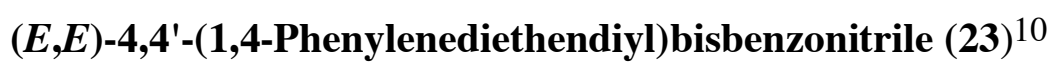

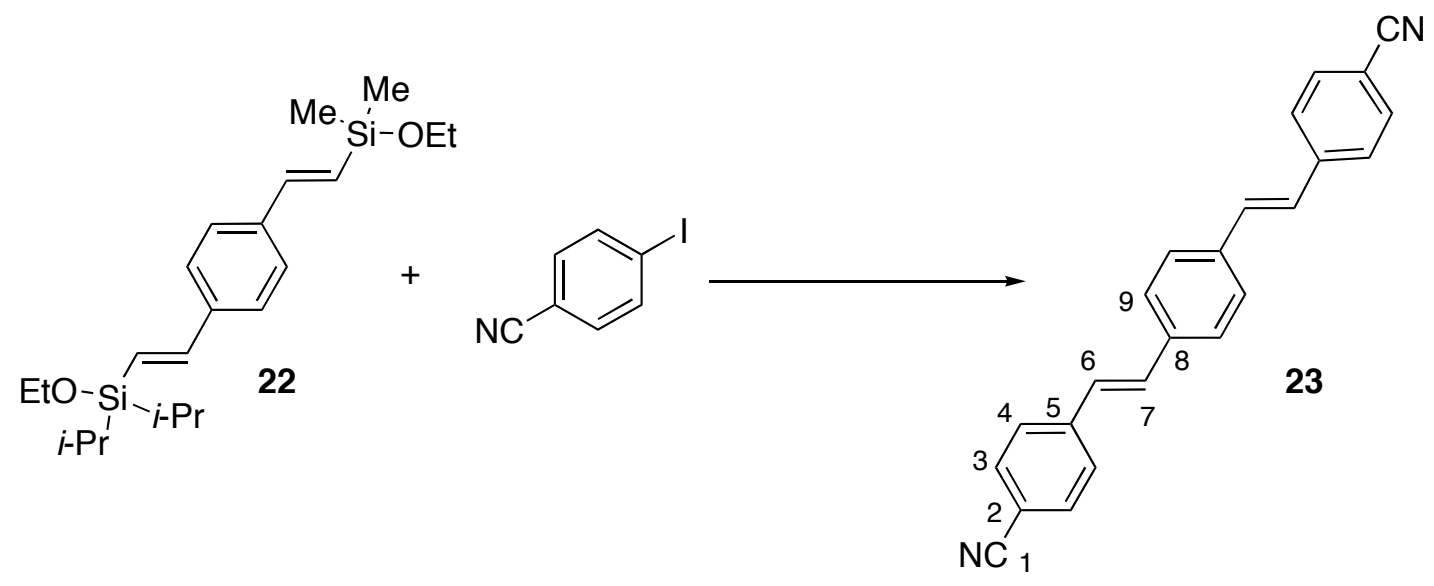

Bis-silane 22 (390 mg, $1.0 \mathrm{mmol}$ ), 4-iodobenzonitrile (458 mg, $2.0 \mathrm{mmol}, 2.0$ equiv), and $(\text { allylPdCl})_{2}(9.3 \mathrm{mg} .0 .025 \mathrm{mmol}, 0.025$ equiv) were dissolved in a solution of TBAF $(4.0 \mathrm{~mL}$, $1.0 \mathrm{mmol}, 1 \mathrm{M}$ in THF, 4 equiv). The reaction mixture was stirred for $6 \mathrm{~h}$ at room temperature. 
The solution was quenched with water $(20 \mathrm{~mL})$ and was extracted with EtOAc $(5 \times 20 \mathrm{~mL})$ and the combined organic phases were washed with brine $(20 \mathrm{~mL})$. The organic layer was dried with $\mathrm{MgSO}_{4}$ (anhydrous) and was filtered. The solvent was then evaporated in vacuo to give a solid which was purified by column chromatography $\left(\mathrm{SiO}_{2}, \mathrm{CH}_{2} \mathrm{Cl}_{2} /\right.$ hexane, 2/1) to afford $262 \mathrm{mg}$ (79\%) of $\mathbf{2 3}$ as yellow solid. ${ }^{10}$

Data for 23:

mp: $\quad 288^{\circ} \mathrm{C}$

${ }^{1} \underline{\mathrm{H} \mathrm{NMR}}: \quad\left(500 \mathrm{MHz}, \mathrm{CDCl}_{3}\right)$

7.65 (d, $J=8.4,4$ H, HC(4)), 7.60 (d, J = 8.4, 4 H, HC(3)), 7.56 (s, 4 H, HC(9)), $7.21(\mathrm{~d}, J=16.3,2 \mathrm{H}, \mathrm{HC}(6)), 7.13(\mathrm{~d}, J=16.3,2 \mathrm{H}, \mathrm{HC}(7))$,

${ }^{13} \mathrm{C} \mathrm{NMR}: \quad\left(126 \mathrm{MHz}, \mathrm{CDCl}_{3}\right)$

$141.8(\mathrm{C}(5)), 136.8(\mathrm{C}(8)), 132.7$ (C(3)), $131.9(\mathrm{C}(6)), 127.6(\mathrm{C}(4)), 127.4(\mathrm{C}(7))$, $127.1(\mathrm{C}(9)), 119.2(\mathrm{C}(1)), 110.9(\mathrm{C}(2))$

TLC: $\quad R_{f} 0.26\left(\mathrm{CH}_{2} \mathrm{Cl}_{2} /\right.$ hexane, $\left.2 / 1\right)\left[\mathrm{UV}+\mathrm{KMnO}_{4}\right]$

\section{4-[2-[4-[2-(2-Methylphenyl)ethenyl]phenyl]ethenyl]benzonitrile (24)}

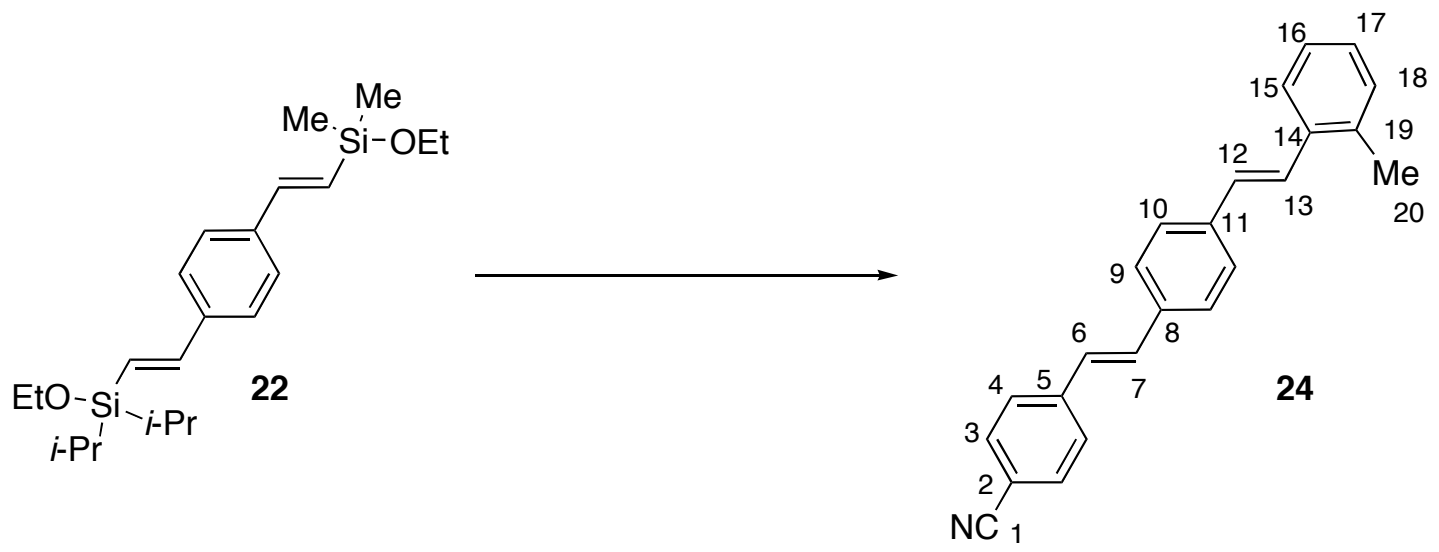

A solution of bis-silane 22 (390 mg, $1.0 \mathrm{mmol})$, 4-iodobenzonitrile (229 mg, $1.0 \mathrm{mmol}$, 1.0 equiv), and (allylPdCl $)_{2}(9.3 \mathrm{mg} .0 .025 \mathrm{mmol}, 0.025$ equiv) in DME (4 mL) was stirred at room temperature for $5 \mathrm{~min}$ and then TMSOK (512 mg, $4.0 \mathrm{mmol}, 4.0$ equiv) was added. The reaction mixture was stirred at room temperature for $6 \mathrm{~h}$ whereupon EtOAc $(20 \mathrm{~mL})$ was added and the reaction was stirred for 10 min further. The reaction mixture was then filtered through a short silica gel column (20 g) and the plug was washed with EtOAc (100 mL) and the solvent was evaporated in vacuo. To the crude product was added 2-iodotoluene (128 $\mu \mathrm{L}, 1.0 \mathrm{mmol}, 1.0$ 
equiv), (allylPdCl$)_{2}(9.3 \mathrm{mg} .0 .025 \mathrm{mmol}, 0.025)$ and a solution of TBAF (3.0 mL, $1 \mathrm{M}$ in THF, $3.0 \mathrm{mmol}, 3.0$ equiv). The reaction mixture was stirred for $4 \mathrm{~h}$ at room temperature, then EtOAc $(25 \mathrm{~mL})$ was added. After stirring 10 min further, the reaction was quenched with water $(25 \mathrm{~mL})$ and extracted with ethyl acetate $(3 \times 25 \mathrm{~mL})$. The combined organic extracts were washed with water $(1 \times 30 \mathrm{~mL})$ and brine $(1 \times 30 \mathrm{~mL})$. The organic layer was dried over $\mathrm{MgSO}_{4}$ (anhydrous) and was filtered. After evaporation of the solvent, the residue was purified by column chromatography $\left(\mathrm{SiO}_{2}\right.$, hexane/ $\left.\mathrm{CH}_{2} \mathrm{Cl}_{2}, 2 / 1\right)$ and sublimed to afford $244 \mathrm{mg}(76 \%)$ of 24 as yellow solid.

Data for 24:

mp: $\quad 294^{\circ} \mathrm{C}($ subl.)

${ }^{1} \underline{\mathrm{H} \text { NMR}}: \quad\left(500 \mathrm{MHz}, \mathrm{CDCl}_{3}\right)$

$7.64(\mathrm{~d}, J=8.5,2 \mathrm{H}, \mathrm{HC}(4)), 7.60(\mathrm{~m}, 3 \mathrm{H}, \mathrm{HC}(3)$ and $\mathrm{HC}(15)), 7.54$ (s, $4 \mathrm{H}$, $\mathrm{HC}(9)$ and $\mathrm{HC}(10)), 7.38(\mathrm{~d}, J=16.4,1 \mathrm{H}, \mathrm{HC}(13)), 7.21(\mathrm{~m}, 4 \mathrm{H}, \mathrm{HC}(6)$, $\mathrm{HC}(16), \mathrm{HC}(17)$, and $\mathrm{HC}(18)), 7.11$ (d, $J=16.3,1 \mathrm{H}, \mathrm{HC}(7)), 7.01$ (d, $J=16.1,1$ $\mathrm{H}, \mathrm{HC}(12)), 2.45$ (s, $3 \mathrm{H}, \mathrm{HC} 20)$ )

${ }^{13} \mathrm{C} \mathrm{NMR}: \quad\left(126 \mathrm{MHz}, \mathrm{CDCl}_{3}\right)$

142.1 (C(5)), 138.3 (C(14)), 136.4 (C(8)), 136.1 (C(11)), 135.8(C(19)), 132.7 (C(3)), 132.2 (C(6)), 130.7 (C(18)), 129.5 (C(17)), 127.9 (C(15)), 127.5 (C(4)), 127.3 (C(7)), 127.2 (C(9)), 127.0 (C(10)), 126.7 (C(16)), 126.5 (C(12)), 125.5 (C(13)), 119.3 (C(1)), 110.7 (C(2)), 20.1 (C(20))

IR: $\quad\left(\mathrm{CHCl}_{3}\right)$

3021 (m), 2227 (s), 1600 (s), 1514 (w), 1460 (w), 1214 (w), $1174(w), 964$ (s)

MS: $\quad(\mathrm{EI}, 70 \mathrm{eV})$

$321\left(\mathrm{M}^{+}, 100\right), 203(13), 157$ (16)

TLC: $\quad R_{f} 0.21\left(\right.$ hexane $\left./ \mathrm{CH}_{2} \mathrm{Cl}_{2}, 2 / 1\right)\left[\mathrm{UV}+\mathrm{KMnO}_{4}\right]$

CG: $\quad t_{\mathrm{R}} 31.24 \min (100 \%)\left(\mathrm{HP} 5\right.$, injector $225^{\circ} \mathrm{C}$, column $\left.275^{\circ} \mathrm{C}, 15 \mathrm{psi}\right)$

HRMS: $\quad$ calc for $\mathrm{C}_{24} \mathrm{H}_{19} \mathrm{~N}_{1}$ : 331.1518; found: 321.1517 
Determination of Response Factors for 1-[(E)-4-(1-Pentenyl)phenyl]ethanone (12) and 1[(E)-4-(1-Heptenyl)phenyl]ethanone (13) with Respect to Naphthalene.

Samples containing various amount of 1-[(E)-4-(1-pentenyl)phenyl]ethanone (12) or 1[(E)-4-(1-heptenyl)phenyl]ethanone (13), and naphthalene were weighed (amounts given below) into small vials. The samples were diluted with $10 \mathrm{~mL}$ of dry THF and were then injected into the GC three times to give the areas indicated below. The response factor for every sample was calculated by

$$
\text { response factor }=\begin{aligned}
& \text { mmol } 12 \times \text { area naphthalene } \\
& \text { area } 12 \times \text { mmol naphthalene }
\end{aligned}
$$

\begin{tabular}{c|c|c|c|c|c|c}
\hline mg naphth & mmol naphth & area naphth & $\mathrm{mg} \mathrm{12}$ & $\mathrm{mmol} 12$ & area 12 & response factor \\
\hline 45.6 & 0.354 & 17650 & 80.5 & 0.428 & 27031 & 0.788 \\
45.6 & 0.354 & 17642 & 80.5 & 0.428 & 26972 & 0.790 \\
45.6 & 0.354 & 17621 & 80.5 & 0.428 & 26752 & 0.795 \\
44.4 & 0.345 & 30424 & 62.9 & 0.334 & 36812 & 0.801 \\
44.4 & 0.345 & 30041 & 62.9 & 0.334 & 36498 & 0.797 \\
44.4 & 0.345 & 30478 & 62.9 & 0.334 & 36904 & 0.800 \\
44.0 & 0.342 & 19642 & 59.1 & 0.314 & 23376 & 0.772 \\
44.0 & 0.342 & 19695 & 59.1 & 0.314 & 23403 & 0.773 \\
44.0 & 0.342 & 19727 & 59.1 & 0.314 & 23490 & 0.771 \\
\hline & & & & & average & 0.787 \\
\hline
\end{tabular}




$$
\text { response factor }=\begin{aligned}
& \text { mmol } 13 \times \text { area naphthalene } \\
& \text { area } 13 \times \text { mmol naphthalene }
\end{aligned}
$$

\begin{tabular}{c|c|c|c|c|c|c}
\hline mg naphth & mmol naphth & area naphth & $\mathrm{mg} \mathrm{13}$ & $\mathrm{mmol} \mathrm{13}$ & area 13 & response factor \\
\hline 48.0 & 0.373 & 28933 & 73.8 & 0.341 & 38879 & 0.681 \\
48.0 & 0.373 & 29169 & 73.8 & 0.341 & 39474 & 0.676 \\
48.0 & 0.373 & 28908 & 73.8 & 0.341 & 39126 & 0.676 \\
45.6 & 0.354 & 17650 & 70.7 & 0.327 & 24499 & 0.665 \\
45.6 & 0.354 & 17642 & 70.7 & 0.327 & 2444 & 0.666 \\
45.6 & 0.354 & 17621 & 70.7 & 0.327 & 24403 & 0.666 \\
44.4 & 0.345 & 30424 & 77.6 & 0.359 & 46491 & 0.681 \\
44.4 & 0.345 & 30041 & 77.6 & 0.359 & 46519 & 0.672 \\
44.4 & 0.345 & 30478 & 77.6 & 0.359 & 46953 & 0.675 \\
\hline
\end{tabular}

Determination of Response Factors for (E)-1-Methyl-2-(1-pentenyl)benzene (16) and (E)-1Methyl-2-(1-heptenyl)benzene (17) with Respect to Naphthalene.

Samples containing various amount of (E)-1-methyl-2-(1-pentenyl)benzene (16), $(E)-1$ methyl-2-(1-heptenyl)benzene (17), and naphthalene were weighed (amounts given below) into small vials. The samples were diluted with $10 \mathrm{~mL}$ dry THF and were then injected into the GC three times to give the areas indicated below. The response factor for every sample was calculated by 
response factor $=\begin{aligned} & \text { mmol } 16 \times \text { area naphthalene } \\ & \text { area } 16 \times \text { mmol naphthalene }\end{aligned}$

\begin{tabular}{c|c|c|c|c|c|c}
\hline mg naphth & mmol naphth & area naphth & $\mathrm{mg} 9$ & $\mathrm{mmol} 9$ & area 9 & response factor \\
\hline 40.5 & 0.315 & 30289 & 44.6 & 0.278 & 31003 & 0.864 \\
40.5 & 0.315 & 29947 & 44.6 & 0.278 & 30419 & 0.871 \\
40.5 & 0.315 & 28763 & 44.6 & 0.278 & 29302 & 0.868 \\
40.3 & 0.313 & 32362 & 52.1 & 0.325 & 39808 & 0.844 \\
40.3 & 0.313 & 41383 & 52.1 & 0.325 & 51256 & 0.838 \\
40.3 & 0.313 & 49832 & 52.1 & 0.325 & 61671 & 0.839 \\
40.3 & 0.313 & 30144 & 49.8 & 0.311 & 34553 & 0.866 \\
40.3 & 0.313 & 44474 & 49.8 & 0.311 & 51428 & 0.858 \\
40.3 & 0.313 & 39946 & 49.8 & 0.311 & 46094 & 0.860 \\
\hline
\end{tabular}

response factor $=\begin{aligned} & \text { mmol } 17 \times \text { area naphthalene } \\ & \text { area } 17 \times \text { mmol naphthalene }\end{aligned}$

\begin{tabular}{c|c|c|c|c|c|c}
\hline mg naphth & mmol naphth & area naphth & $\mathrm{mg} \mathrm{17}$ & $\mathrm{mmol} \mathrm{17}$ & area 17 & Response factor \\
\hline 41.9 & 0.325 & 36983 & 57.1 & 0.303 & 48111 & 0.716 \\
41.9 & 0.325 & 39439 & 57.1 & 0.303 & 51425 & 0.715 \\
41.9 & 0.325 & 40007 & 57.1 & 0.303 & 52262 & 0.713 \\
40.5 & 0.315 & 32362 & 36.7 & 0.195 & 28947 & 0.693 \\
40.5 & 0.315 & 41383 & 36.7 & 0.195 & 37631 & 0.681 \\
40.5 & 0.315 & 49832 & 36.7 & 0.195 & 45294 & 0.682 \\
40.3 & 0.313 & 30289 & 18.5 & 0.098 & 13049 & 0.728 \\
40.3 & 0.313 & 29947 & 18.5 & 0.098 & 12827 & 0.733 \\
40.3 & 0.313 & 28763 & 18.5 & 0.098 & 12351 & 0.731 \\
\hline
\end{tabular}


Determination of Response Factors for $(E)$-1-Methoxy-4-(1-pentenyl)benzene (14) and $(E)$ -

\section{1-Methoxy-4-(1-heptenyl)benzene (15) with Respect to Naphthalene.}

Samples containing various amount of $(E)$-1-methoxy-4-(1-pentenyl)benzene (14) or $(E)$ 1-methoxy-4-(1-heptenyl)benzene (15), and naphthalene were weighed (amounts given below) into small vials. The samples were diluted with $10 \mathrm{~mL}$ dry $\mathrm{THF}$ and were then injected into the GC three times to give the areas indicated bellow. The response factor for every sample was calculated by

$$
\text { response factor }=\begin{aligned}
& \text { mmol } 14 \times \text { area naphthalene } \\
& \text { area } 14 \times \text { mmol naphthalene }
\end{aligned}
$$

\begin{tabular}{c|c|c|c|c|c|c}
\hline mg naphth & mmol naphth & area naphth & $\mathrm{mg} \mathrm{14}$ & $\mathrm{mmol} 14$ & area 14 & response factor \\
\hline 46.7 & 0.365 & 72420 & 53.5 & 0.304 & 62181 & 0.970 \\
46.7 & 0.365 & 71762 & 53.5 & 0.304 & 61892 & 0.965 \\
46.7 & 0.365 & 72116 & 53.5 & 0.304 & 62232 & 0.965 \\
46.3 & 0.363 & 47337 & 42.9 & 0.244 & 33038 & 0.964 \\
46.3 & 0.363 & 47327 & 42.9 & 0.244 & 33121 & 0.961 \\
46.3 & 0.363 & 47095 & 42.9 & 0.244 & 32856 & 0.964 \\
38.3 & 0.299 & 35469 & 59.1 & 0.314 & 26951 & 0.974 \\
38.3 & 0.299 & 35430 & 59.1 & 0.314 & 27081 & 0.968 \\
38.3 & 0.299 & 35546 & 59.1 & 0.314 & 26987 & 0.975 \\
\hline & & & & & average & 0.967 \\
\hline
\end{tabular}




\begin{tabular}{|c|c|c|c|c|c|c|}
\hline \multicolumn{7}{|c|}{ response factor $=$} \\
\hline mg naphth & mmol naphth & area naphth & $\mathrm{mg} 15$ & $\mathrm{mmol} 15$ & area 15 & response factor \\
\hline 52.5 & 0.410 & 60006 & 61.2 & 0.300 & 54756 & 0.801 \\
\hline 52.5 & 0.410 & 60681 & 61.2 & 0.300 & 55506 & 0.799 \\
\hline 52.5 & 0.410 & 60742 & 61.2 & 0.300 & 55982 & 0.794 \\
\hline 46.7 & 0.365 & 72116 & 79.7 & 0.390 & 97750 & 0.789 \\
\hline 46.7 & 0.365 & 71762 & 79.7 & 0.390 & 97148 & 0.790 \\
\hline 46.7 & 0.365 & 72420 & 79.7 & 0.390 & 97336 & 0.796 \\
\hline 46.4 & 0.363 & 47095 & 56.0 & 0.363 & 44820 & 0.795 \\
\hline 46.4 & 0.363 & 47327 & 56.0 & 0.363 & 45218 & 0.792 \\
\hline \multirow[t]{2}{*}{46.4} & 0.363 & 47337 & 56.0 & 0.363 & 45330 & 0.790 \\
\hline & & & & & average & 0.794 \\
\hline
\end{tabular}




\section{Competition Experiments from Table 1 (Carbon Substituent Effects).}

\section{Competition Experiments with 4-Iodoacetophenone. General Procedure I.}

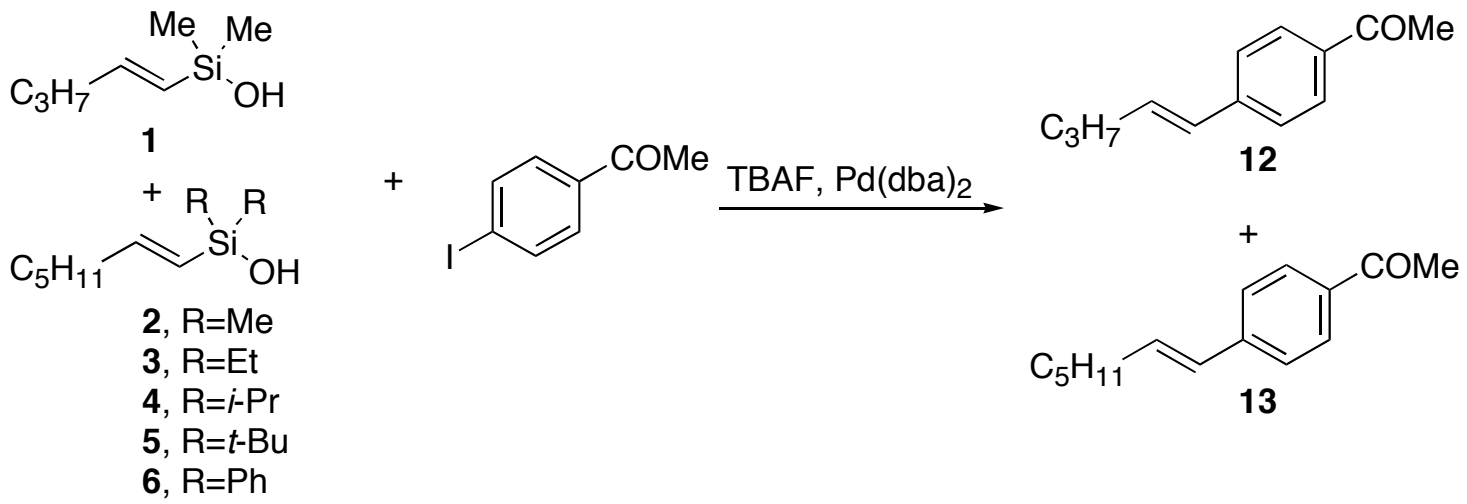

(E)-Dimethyl-(1-pentenyl)silanol (1), together with one of the other 1-heptenylsilanols (2-6), naphthalene, and $\operatorname{Pd}(\mathrm{dba})_{2}$ were dissolved in a TBAF solution (1.0 $\mathrm{M}$ in THF) in a flamedried, 5-mL, 2-neck, round-bottomed flask under $\mathrm{N}_{2}$. The 4-iodoacetophenone was then added slowly to maintain an internal temperature $<30^{\circ} \mathrm{C}$. After the reaction was complete, determined by TLC, $25-\mu \mathrm{L}$ samples were taken via syringe. The sample aliquots were filtered through a plug of silica gel washing with hexane/ethyl acetate, $4 / 1$ to achieve a total sample volume of $\sim 2$ $\mathrm{mL}$. These samples were then subjected to GC analysis. The reaction mixture was filtered through a plug of silica gel ( 15 g). The plug was washed with hexane/ethyl acetate, 4/1, (100 $\mathrm{mL}$ ), and the solvent was evaporated in vacuo. The residue was purified by column chromatography (Reverse Phase $\mathrm{C} 18, \mathrm{MeOH} / \mathrm{H}_{2} \mathrm{O}, 9 / 1$ ) to afford the 12, and $\mathbf{1 3}$ which were further purified by Kugelrohr distillation.

\section{Competition of $(E)$-Dimethyl-(1-pentenyl)silanol (1) vs (E)-Dimethyl-(1 heptenyl)silanol (2) with 4-Iodoacetophenone}

Following General Procedure I, 1 (83.9 mg, $0.58 \mathrm{mmol}), 2$ (97.1 mg, $0.56 \mathrm{mmol})$, naphthalene $(67.7 \mathrm{mg}, 0.53 \mathrm{mmol}), \mathrm{TBAF}(2.0 \mathrm{~mL}, 2.0 \mathrm{mmol}, 1.0 \mathrm{M}$ in THF), Pd(dba)2 (14.4 $\mathrm{mg} 0.025 \mathrm{mmol})$, and 4-iodoacetophenone $(123.0 \mathrm{mg}, 0.50 \mathrm{mmol})$ was stirred at room temperature for $10 \mathrm{~min}$ and then sample aliquots were taken and reaction was worked up to afford $48 \mathrm{mg}(51 \%)$ of $\mathbf{1 2}$ and $47 \mathrm{mg}(43 \%)$ of 13. GC analysis of samples showed a 12/13 ratio of $52.2 / 47.8$. 
GC Data:

\begin{tabular}{|c|c|c|c|c|c|}
\hline & area naphth & area 12 & area 13 & response factor $12 / 13$ & ratio $12 / 13 \times 100$ \\
\hline sample1 & 5546 & 4247 & 4396 & $0.78 / 0.67$ & $53.1 / 46.9$ \\
\hline sample2 & 6125 & 7688 & 8524 & $0.78 / 0.67$ & $51.3 / 48.7$ \\
\hline average & 5836 & 5968 & 6460 & $0.78 / 0.67$ & $52.2 / 47.8$ \\
\hline
\end{tabular}

Competition of (E)-Dimethyl-(1-pentenyl)silanol (1) vs (E)-Dimethyl-(1-heptenyl)silanol (2) with 4-Iodoacetophenone

Following General Procedure I, 1 (71.8 mg, $0.50 \mathrm{mmol}), 2(85.9 \mathrm{mg}, 0.50 \mathrm{mmol})$, naphthalene (65.3 mg, $0.51 \mathrm{mmol})$, TBAF (2.0 mL, $2.0 \mathrm{mmol}, 1.0 \mathrm{M}$ in THF), Pd(dba) 2 (14.4 $\mathrm{mg} 0.025 \mathrm{mmol})$, and 4-iodoacetophenone $(123.0 \mathrm{mg}, 0.50 \mathrm{mmol})$ was stirred at room temperature for $10 \mathrm{~min}$ and then sample aliquots were taken and reaction was worked up to afford $43 \mathrm{mg}$ (46\%) of $\mathbf{1 2}$ and $48 \mathrm{mg}(44 \%)$ of $\mathbf{1 3}$. GC analysis of samples showed a $\mathbf{1 2} / \mathbf{1 3}$ ratio of 50.1/49.9.

GC Data:

\begin{tabular}{l|c|c|c|c|c|}
\cline { 2 - 6 } & area naphth & area 12 & area 13 & response factor $12 / 13$ & ratio $12 / 13 \times 100$ \\
\hline sample 1 & 15309 & 10206 & 11863 & $0.78 / 0.67$ & $50.2 / 49.84$ \\
\hline
\end{tabular}

\section{Competition of (E)-Dimethyl-(1-pentenyl)silanol (1) vs (E)-Dimethyl-(1-heptenyl)silanol (2) with 4-Iodoacetophenone}

Following General Procedure I, 1 (72.1 mg, $0.50 \mathrm{mmol}), 2(86.2 \mathrm{mg}, 0.50 \mathrm{mmol})$, naphthalene (64.5 mg, $0.50 \mathrm{mmol})$, TBAF (2.0 mL, $2.0 \mathrm{mmol}, 1.0 \mathrm{M}$ in THF), Pd(dba) 2 (14.4 $\mathrm{mg} 0.025 \mathrm{mmol})$, and 4-iodoacetophenone $(123.0 \mathrm{mg}, 0.50 \mathrm{mmol})$ was stirred at room temperature for $10 \mathrm{~min}$ and then sample aliquots were taken and reaction was worked up to afford $40 \mathrm{mg}$ (43\%) of $\mathbf{1 2}$ and $46 \mathrm{mg}$ (43\%) of $\mathbf{1 3}$. GC analysis of samples showed a $\mathbf{1 2} / \mathbf{1 3}$ ratio of $49.9 / 50.1$.

GC Data:

\begin{tabular}{l|c|c|c|c|c|}
\cline { 2 - 6 } & area naphth & area 12 & area 13 & response factor 12/13 & ratio 12/13×100 \\
\hline sample 1 & 6207 & 3990 & 4598 & $0.78 / 0.67$ & $50.4 / 49.6$ \\
\hline sample 2 & 8196 & 4404 & 5257 & $0.78 / 0.67$ & $49.5 / 50.1$ \\
\hline average & 7202 & 4197 & 4928 & $0.78 / 0.67$ & $49.9 / 50.1$ \\
\hline
\end{tabular}




\section{Competition of $(E)$-Dimethyl-(1-pentenyl)silanol (1) vs (E)-Dimethyl-(1-heptenyl)silanol (2)} with 4-Iodoacetophenone

Following General Procedure I, 1 (71.7 mg, $0.50 \mathrm{mmol}), 2$ (86.2 mg, $0.50 \mathrm{mmol})$, naphthalene (64.2 mg, $0.50 \mathrm{mmol})$, TBAF (2.0 mL, $2.0 \mathrm{mmol}, 1.0 \mathrm{M}$ in THF), Pd(dba)2 (14.2 $\mathrm{mg} 0.025 \mathrm{mmol})$, and 4-iodoacetophenone $(123.1 \mathrm{mg}, 0.50 \mathrm{mmol})$ was stirred at room temperature for $10 \mathrm{~min}$ and then sample aliquots were taken and reaction was worked up to afford $41 \mathrm{mg}(44 \%)$ of $\mathbf{1 2}$ and $47 \mathrm{mg}(43 \%)$ of $\mathbf{1 3}$. GC analysis of samples showed a 12/13 ratio of 50.9/49.1.

GC Data:

\begin{tabular}{l|c|c|c|c|c|}
\cline { 2 - 6 } & area naphth & area 12 & area 13 & response factor $12 / 13$ & ratio 12/13×100 \\
\hline sample 1 & 7691 & 4788 & 5450 & $0.78 / 0.67$ & $50.7 / 49.3$ \\
\hline sample 2 & 7560 & 4886 & 5463 & $0.78 / 0.67$ & $51.1 / 48.9$ \\
\hline average & 7626 & 4837 & 5457 & $0.78 / 0.67$ & $50.9 / 49.1$ \\
\hline
\end{tabular}

\section{Competition of $(E)$-Dimethyl-(1-pentenyl)silanol (1) vs (E)-Diethyl-(1-heptenyl)silanol (3)} with 4-Iodoacetophenone

Following General Procedure I, 1 (71.4 mg, $0.50 \mathrm{mmol}), 3$ (100.4 mg, $0.50 \mathrm{mmol})$, naphthalene (64.3 mg, $0.50 \mathrm{mmol})$, TBAF (2.0 mL, $2.0 \mathrm{mmol}, 1.0 \mathrm{M}$ in THF), Pd(dba)2 (14.4 $\mathrm{mg} 0.025 \mathrm{mmol})$, and 4-iodoacetophenone $(123.0 \mathrm{mg}, 0.50 \mathrm{mmol})$ was stirred at room temperature for $10 \mathrm{~min}$ and then sample aliquots were taken and reaction was worked up to afford $49 \mathrm{mg}(52 \%)$ of $\mathbf{1 2}$ and $40 \mathrm{mg}(37 \%)$ of $\mathbf{1 3}$. GC analysis of samples showed a 12/13 ratio of $56.7 / 43.3$.

GC Data:

\begin{tabular}{|c|c|c|c|c|c|}
\hline & area naphth & area 12 & area 13 & response factor $12 / 13$ & ratio $12 / 13 \times 100$ \\
\hline sample 1 & 14122 & 9596 & 8559 & $0.78 / 0.67$ & $56.7 / 43.3$ \\
\hline sample 2 & 18001 & 12673 & 11386 & $0.78 / 0.67$ & $56.6 / 43.4$ \\
\hline average & 16062 & 11135 & 9973 & $0.78 / 0.67$ & $56.7 / 43.3$ \\
\hline
\end{tabular}

\section{Competition of $(E)$-Dimethyl-(1-pentenyl)silanol (1) vs (E)-Diethyl-(1-heptenyl)silanol (3) with 4-Iodoacetophenone}

Following General Procedure I, 1 (71.9 mg, $0.50 \mathrm{mmol}), 3$ (100.4 mg, $0.50 \mathrm{mmol})$, naphthalene (64.6 mg, $0.50 \mathrm{mmol})$, TBAF (2.0 mL, $2.0 \mathrm{mmol}, 1.0 \mathrm{M}$ in THF), Pd(dba)2 (14.4 
mg $0.025 \mathrm{mmol})$, and 4-iodoacetophenone $(123.0 \mathrm{mg}, 0.50 \mathrm{mmol})$ was stirred at room temperature for $10 \mathrm{~min}$ and then sample aliquots were taken and reaction was worked up to afford $49 \mathrm{mg}(52 \%)$ of $\mathbf{1 2}$ and $44 \mathrm{mg}(41 \%)$ of $\mathbf{1 3}$. GC analysis of samples showed a $\mathbf{1 2} / \mathbf{1 3}$ ratio of 56.6/43.4.

GC Data:

\begin{tabular}{|c|c|c|c|c|c|}
\hline & area naphth & area 12 & area 13 & response factor $12 / 13$ & ratio $12 / 13 \times 100$ \\
\hline sample 1 & 12519 & 8940 & 8231 & $0.78 / 0.67$ & $55.9 / 44.1$ \\
\hline sample 2 & 8857 & 6408 & 5624 & $0.78 / 0.67$ & $57.1 / 42.9$ \\
\hline average & 10688 & 7674 & 6928 & $0.78 / 0.67$ & $56.6 / 43.4$ \\
\hline
\end{tabular}

\section{Competition of (E)-Dimethyl-(1-pentenyl)silanol (1) vs. (E)-Di-isopropyl-(1-heptenyl)silanol} (4) with 4-Iodoacetophenone

Following General Procedure I, 1 (71.3 mg, $0.49 \mathrm{mmol}), 4$ (115.1 mg, $0.50 \mathrm{mmol})$, naphthalene $(65.3 \mathrm{mg}, 0.51 \mathrm{mmol})$, TBAF $(2.0 \mathrm{~mL}, 2.0 \mathrm{mmol}, 1 \mathrm{M}$ in THF), Pd(dba) 2 (14.4 mg $0.025 \mathrm{mmol})$, and 4-iodoacetophenone $(123.0 \mathrm{mg}, 0.50 \mathrm{mmol})$ was stirred at room temperature for $10 \mathrm{~min}$ and then sample aliquots were taken and reaction was worked up to afford $51 \mathrm{mg}$ (54\%) of $\mathbf{1 2}$ and $41 \mathrm{mg}$ (38\%) of 13. GC analysis of samples showed a 12/13 ratio of 59.8/40.2.

GC Data:

\begin{tabular}{l|c|c|c|c|c|}
\cline { 2 - 6 } & area naphth & area 12 & area 13 & response factor 12/13 & ratio 12/13×100 \\
\hline sample 1 & 8312 & 6852 & 5043 & $0.78 / 0.67$ & $61.4 / 38.6$ \\
\hline sample 2 & 17074 & 11744 & 9869 & $0.78 / 0.67$ & $58.2 / 41.8$ \\
\hline average & 12693 & 9298 & 7456 & $0.78 / 0.67$ & $59.8 / 40.2$ \\
\hline
\end{tabular}

\section{Competition of $(E)$-Dimethyl-(1-pentenyl)silanol (1) vs (E)-Di-(1-methylethyl)-(1-heptenyl)-} silanol (4) with 4-Iodoacetophenone

Following General Procedure I, 1 (72.4 mg, $0.50 \mathrm{mmol}), 4$ (114.5 mg, $0.50 \mathrm{mmol})$, naphthalene (66.4 mg, $0.52 \mathrm{mmol})$, TBAF (2.0 mL, $2.0 \mathrm{mmol}, 1.0 \mathrm{M}$ in THF), Pd(dba)2 (14.4 $\mathrm{mg} 0.025 \mathrm{mmol})$, and 4-iodoacetophenone $(123.0 \mathrm{mg}, 0.50 \mathrm{mmol})$ was stirred at room temperature for $10 \mathrm{~min}$ and then sample aliquots were taken and reaction was worked up to afford $49 \mathrm{mg}(52 \%)$ of $\mathbf{1 2}$ and $37 \mathrm{mg}(34 \%)$ of 13. GC analysis of samples showed a 12/13 ratio of $59.9 / 40.1$. 
GC Data:

\begin{tabular}{l|c|c|c|c|c|}
\cline { 2 - 6 } & area naphth & area 12 & area 13 & response factor 12/13 & ratio 12/13×100 \\
\hline sample 1 & 9300 & 6470 & 5213 & $0.78 / 0.67$ & $59.2 / 40.8$ \\
\hline sample 2 & 8435 & 6870 & 5238 & $0.78 / 0.67$ & $60.5 / 39.5$ \\
\hline average & 8868 & 6670 & 5226 & $0.78 / 0.67$ & $59.9 / 40.1$ \\
\hline
\end{tabular}

Competition of (E)-Dimethyl-(1-pentenyl)silanol (1) vs (E)-Di-(1,1-dimethylethyl)-(1heptenyl)silanol (5) with 4-Iodoacetophenone

Following General Procedure I, 1 (71.4 mg, $0.50 \mathrm{mmol}), 5(127.9 \mathrm{mg}, 0.50 \mathrm{mmol})$, naphthalene (63.7 mg, $0.50 \mathrm{mmol})$, TBAF (2.0 mL, $2.0 \mathrm{mmol}, 1.0 \mathrm{M}$ in THF), Pd(dba) 2 (14.4 $\mathrm{mg} 0.025 \mathrm{mmol})$, and 4-iodoacetophenone $(123.0 \mathrm{mg}, 0.50 \mathrm{mmol})$ was stirred at room temperature for $10 \mathrm{~min}$ and then sample aliquots were taken and reaction was worked up to afford $78.2 \mathrm{mg}$ (83\%) of $\mathbf{1 2}$. GC analysis of samples showed a $\mathbf{1 2 / 1 3}$ ratio of 96.7/3.3.

GC Data:

\begin{tabular}{l|c|c|c|c|c|}
\cline { 2 - 6 } & area naphth & area 12 & area 13 & response factor 12/13 & ratio 12/13×100 \\
\hline sample 1 & 9077 & 10986 & 465 & $0.78 / 0.67$ & $96.5 / 3.5$ \\
\hline sample 2 & 12930 & 15354 & 581 & $0.78 / 0.67$ & $96.8 / 3.2$ \\
\hline average & 11004 & 13170 & 523 & $0.78 / 0.67$ & $96.7 / 3.3$ \\
\hline
\end{tabular}

\section{Competition of (E)-Dimethyl-(1-pentenyl)silanol (1) vs (E)-Di-(1,1-dimethylethyl)-(1- heptenyl)silanol (5) with 4-Iodoacetophenone}

Following General Procedure I, 1 (72.0 mg, $0.50 \mathrm{mmol}), 5(130.6 \mathrm{mg}, 0.51 \mathrm{mmol})$, naphthalene (65.0 mg, $0.51 \mathrm{mmol}$ ), TBAF (2.0 mL, $2.0 \mathrm{mmol}, 1.0 \mathrm{M}$ in THF), Pd(dba) 2 (14.4 $\mathrm{mg} 0.025 \mathrm{mmol})$, and 4-iodoacetophenone $(123.0 \mathrm{mg}, 0.50 \mathrm{mmol})$ was stirred at room temperature for $10 \mathrm{~min}$ and then sample aliquots were taken and reaction was worked up to afford $77.9 \mathrm{mg}(83 \%)$ of $\mathbf{1 2}$. GC analysis of samples showed a $\mathbf{1 2 / 1 3}$ ratio of 96.1/3.9.

GC Data:

\begin{tabular}{|c|c|c|c|c|c|}
\hline & area naphth & area 12 & area 13 & response factor $12 / 13$ & ratio $12 / 13 \times 100$ \\
\hline sample 1 & 12043 & 13323 & 727 & $0.78 / 0.67$ & $95.5 / 4.5$ \\
\hline sample 2 & 16246 & 18945 & 748 & $0.78 / 0.67$ & $96.7 / 3.3$ \\
\hline average & 14145 & 16134 & 738 & $0.78 / 0.67$ & $96.1 / 3.9$ \\
\hline
\end{tabular}


Competition of $(E)$-Dimethyl-(1-pentenyl)silanol (1) vs (E)-Diphenyl-(1-heptenyl)silanol (6) with 4-Iodoacetophenone

Following General Procedure I, 1 (72.7 mg, $0.50 \mathrm{mmol})$, 6 (148.5 mg, $0.50 \mathrm{mmol})$, naphthalene (64.0 mg, $0.50 \mathrm{mmol})$, TBAF (2.0 mL, $2.0 \mathrm{mmol}, 1.0 \mathrm{M}$ in THF), Pd(dba)2 (14.4 $\mathrm{mg} 0.025 \mathrm{mmol})$, and 4-iodoacetophenone $(123.1 \mathrm{mg}, 0.50 \mathrm{mmol})$ was stirred at room temperature for $10 \mathrm{~min}$ and then sample aliquots were taken and reaction was worked up to afford $42 \mathrm{mg}$ (44\%) of $\mathbf{1 2}$ and $47 \mathrm{mg}(43 \%)$ of $\mathbf{1 3}$. GC analysis of samples showed a 12/13 ratio of 50.0/50.0.

GC Data:

\begin{tabular}{l|c|c|c|c|c|}
\cline { 2 - 6 } & area naphth & area 12 & area 13 & response factor 12/13 & ratio 12/13×100 \\
\hline sample 1 & 6743 & 4314 & 5140 & $0.78 / 0.67$ & $49.5 / 50.5$ \\
\hline sample 2 & 11856 & 7302 & 7979 & $0.78 / 0.67$ & $51.7 / 48.3$ \\
\hline sample 3 & 13140 & 9921 & 12249 & $0.78 / 0.67$ & $48.7 / 51.3$ \\
\hline average & 10580 & 7179 & 8456 & $0.78 / 0.67$ & $50.0 / 50.0$ \\
\hline
\end{tabular}

Competition of $(E)$-Dimethyl-(1-pentenyl)silanol (1) vs (E)-Diphenyl-(1-heptenyl)silanol (6) with 4-Iodoacetophenone

Following General Procedure I, 1 (72.6 mg, $0.50 \mathrm{mmol}), 6$ (148.7 mg, $0.50 \mathrm{mmol})$, naphthalene (64.5 mg, $0.50 \mathrm{mmol})$, TBAF (2.0 mL, $2.0 \mathrm{mmol}, 1.0 \mathrm{M}$ in THF), Pd(dba)2 (14.4 $\mathrm{mg} 0.025 \mathrm{mmol})$, and 4-iodoacetophenone $(123.2 \mathrm{mg}, 0.50 \mathrm{mmol})$ was stirred at room temperature for $10 \mathrm{~min}$ and then sample aliquots were taken and reaction was worked up to afford $41 \mathrm{mg}(44 \%)$ of $\mathbf{1 2}$ and $47 \mathrm{mg}(43 \%)$ of $\mathbf{1 3}$. GC analysis of samples showed a 12/13 ratio of 48.5/51.5.

GC Data:

\begin{tabular}{|c|c|c|c|c|c|}
\hline & area naphth & area 12 & area 13 & response factor $12 / 13$ & ratio $12 / 13 \times 100$ \\
\hline sample 1 & 15024 & 10671 & 13154 & $0.78 / 0.67$ & $48.7 / 51.3$ \\
\hline sample 2 & 20718 & 15021 & 18537 & $0.78 / 0.67$ & $48.7 / 51.3$ \\
\hline sample 3 & 17660 & 12865 & 16263 & $0.78 / 0.67$ & $48.0 / 51.9$ \\
\hline average & 17801 & 12852 & 15985 & $0.78 / 0.67$ & $48.5 / 51.5$ \\
\hline
\end{tabular}


Competition of $(E)$-Dimethyl-(1-pentenyl)silanol (1) vs (E)-Diphenyl-(1-heptenyl)silanol (6) with 4-Iodoacetophenone

Following General Procedure I, 1 (86 $\mu \mathrm{L}, 0.50 \mathrm{mmol}), 6$ (148.4 mg, $0.50 \mathrm{mmol})$, naphthalene (65.9 mg, $0.51 \mathrm{mmol})$, TBAF (2.0 mL, $2.0 \mathrm{mmol}, 1.0 \mathrm{M}$ in THF), Pd(dba) 2 (14.4 $\mathrm{mg} 0.025 \mathrm{mmol})$, and 4-iodoacetophenone $(123.0 \mathrm{mg}, 0.50 \mathrm{mmol})$ was stirred at room temperature for $10 \mathrm{~min}$ and then sample aliquots were taken and reaction was worked up to afford $43 \mathrm{mg}(46 \%)$ of $\mathbf{1 2}$ and $48 \mathrm{mg}(45 \%)$ of $\mathbf{1 3}$. GC analysis of samples showed a 12/13 ratio of $48.4 / 51.6$.

GC Data:

\begin{tabular}{l|c|c|c|c|c|}
\cline { 2 - 6 } & area naphth & area 12 & area 13 & response factor 12/13 & ratio 12/13×100 \\
\hline sample 1 & 8544 & 6339 & 7850 & $0.78 / 0.67$ & $48.6 / 51.4$ \\
\hline sample 2 & 9659 & 7489 & 8883 & $0.78 / 0.67$ & $49.7 / 50.3$ \\
\hline sample 3 & 8171 & 6224 & 8210 & $0.78 / 0.67$ & $47.0 / 53.0$ \\
\hline average & 8791 & 6684 & 8314 & $0.78 / 0.67$ & $48.4 / 51.6$ \\
\hline
\end{tabular}

Competition of $(E)$-Dimethyl-(1-pentenyl)silanol (1) vs (E)-Diphenyl-(1-heptenyl)silanol (6) with 4-Iodoacetophenone

Following General Procedure I, $1(86 \mu \mathrm{L}, 0.50 \mathrm{mmol}), 6$ (148.6 mg, $0.50 \mathrm{mmol})$, naphthalene (65.3 mg, $0.51 \mathrm{mmol})$, TBAF (2.0 mL, $2.0 \mathrm{mmol}, 1.0 \mathrm{M}$ in THF), Pd(dba)2 (14.4 $\mathrm{mg} 0.025 \mathrm{mmol})$, and 4-iodoacetophenone $(123.0 \mathrm{mg}, 0.50 \mathrm{mmol})$ was stirred at room temperature for $10 \mathrm{~min}$ and then sample aliquots were taken and reaction was worked up to afford $42 \mathrm{mg}(45 \%)$ of $\mathbf{1 2}$ and $48 \mathrm{mg}(44 \%)$ of $\mathbf{1 3}$. GC analysis of samples showed a 12/13 ratio of 51.1/48.9.

GC Data:

\begin{tabular}{l|c|c|c|c|c|}
\cline { 2 - 6 } & area naphth & area 12 & area 13 & response factor 12/13 & ratio 12/13×100 \\
\hline sample 1 & 9901 & 9577 & 10105 & $0.78 / 0.67$ & $52.6 / 47.4$ \\
\hline sample 2 & 7927 & 7712 & 8825 & $0.78 / 0.67$ & $50.5 / 49.5$ \\
\hline sample 3 & 9563 & 6545 & 7641 & $0.78 / 0.67$ & $50.0 / 50.0$ \\
\hline average & 9130 & 7945 & 8857 & $0.78 / 0.67$ & $51.1 / 48.9$ \\
\hline
\end{tabular}




\section{Competition Experiments with 4-Iodoanisole. General Procedure II.}

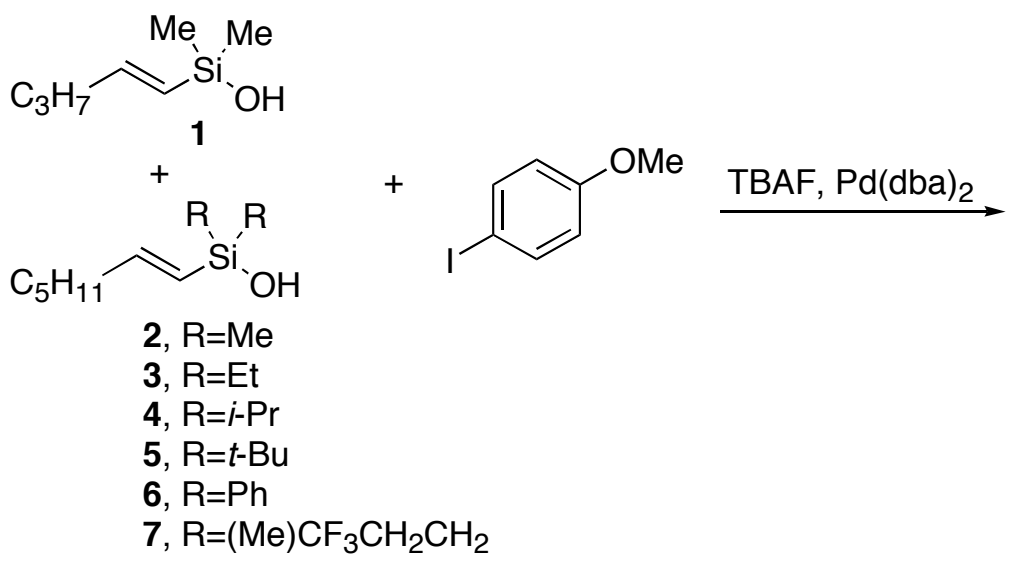<smiles></smiles>

15

A flame-dried, 5-mL, 2-neck, round-bottomed flask under $\mathrm{N}_{2}$ was charged with $(E)$ dimethyl-(1-pentenyl)silanol (1), one of the 1-heptenylsilanols (2-6) and 4-iodoanisole. THF solutions of naphthalene $(0.25 \mathrm{M})$ and TBAF $(1.0 \mathrm{M})$ were added next followed by $\operatorname{Pd}(\mathrm{dba})_{2}$. After 30 min two $25 \mu \mathrm{L}$ samples were taken via syringe. The sample aliquots were filtered through a plug of silica gel washing with $\mathrm{Et}_{2} \mathrm{O}$ to achieve a total sample volume of $\sim 2 \mathrm{~mL}$. These samples were then subjected to GC analysis. Reactions were performed in duplicate.

\section{Competition of $(E)$-Dimethyl-(1-pentenyl)silanol (1) vs (E)-Dimethyl-(1 heptenyl)silanol (2)} with 4-Iodoanisole

Following General Procedure II, 1 (28.8 mg, $0.20 \mathrm{mmol}), 2$ (34.2 mg, $0.20 \mathrm{mmol})$, 4iodoanisole $(46.8 \mathrm{mg}, 0.20 \mathrm{mmol})$, a solution of naphthalene $(0.8 \mathrm{~mL}, 0.20 \mathrm{mmol}, 0.25 \mathrm{M}$ in $\mathrm{THF})$ and TBAF (0.8 mL, $0.80 \mathrm{mmol}, 1.0 \mathrm{M}$ in THF) and $\mathrm{Pd}(\mathrm{dba})_{2}(5.8 \mathrm{mg} 0.01 \mathrm{mmol})$ were stirred at room temperature for $1 \mathrm{~h}$ and then two sample aliquots were taken and analyzed twice on GC. GC analysis of samples showed a 14/15 ratio of 50.6/49.4.

GC Data:

\begin{tabular}{l|c|c|c|c|c|}
\cline { 2 - 6 } reaction 1 & area naphth & area 14 & area 15 & response factor 14/15 & ratio 14/15×100 \\
\hline sample 1 & 17073 & 6579 & 7905 & $0.97 / 0.79$ & $50.3 / 49.7$ \\
\hline & 15511 & 7350 & 9663 & $0.97 / 0.79$ & $48.1 / 51.9$ \\
\hline sample 2 & 17040 & 6385 & 7683 & $0.97 / 0.79$ & $50.3 / 49.7$ \\
\hline & 15464 & 6638 & 7780 & $0.97 / 0.79$ & $50.9 / 49.1$ \\
\hline average & 16272 & 6738 & 8258 & $0.97 / 0.79$ & $49.9 / 50.1$ \\
\hline
\end{tabular}




\begin{tabular}{l|c|c|c|c|c|}
\cline { 2 - 6 } reaction 2 & area naphth & area 14 & area 15 & response factor 14/15 & ratio 14/15×100 \\
\hline sample 1 & 15930 & 6665 & 7546 & $0.97 / 0.79$ & $51.8 / 48.2$ \\
\hline & 15320 & 6589 & 7544 & $0.97 / 0.79$ & $51.5 / 48.5$ \\
\hline sample 2 & 18365 & 7931 & 8950 & $0.97 / 0.79$ & $51.9 / 48.1$ \\
\hline & 19179 & 8006 & 9632 & $0.97 / 0.79$ & $50.3 / 49.7$ \\
\hline average & 17199 & 7298 & 8418 & $0.97 / 0.79$ & $51.4 / 48.6$ \\
\hline
\end{tabular}

Competition of $(E)$-Dimethyl-(1-pentenyl)silanol (1) vs (E)-Diethyl-(1 heptenyl)silanol (3) with 4-Iodoanisole

Following General Procedure II, 1 (28.8 mg, $0.20 \mathrm{mmol}), 3$ (40.0 mg, $0.20 \mathrm{mmol}), 4-$ iodoanisole $(46.8 \mathrm{mg}, 0.20 \mathrm{mmol})$, a solution of naphthalene $(0.8 \mathrm{~mL}, 0.20 \mathrm{mmol}, 0.25 \mathrm{M}$ in THF) and TBAF (0.8 mL, $0.80 \mathrm{mmol}, 1.0 \mathrm{M}$ in THF) and Pd(dba) $2(5.8 \mathrm{mg} 0.01 \mathrm{mmol})$ was stirred at room temperature for $1 \mathrm{~h}$ and then two sample aliquots were taken and analyzed twice on GC. GC analysis of samples showed a 14/15 ratio of 53.3/46.7.

GC Data:

\begin{tabular}{l|c|c|c|c|c|}
\cline { 2 - 6 } reaction 1 & area naphth & area 14 & area 15 & response factor 14/15 & ratio 14/15×100 \\
\hline sample 1 & 52775 & 28507 & 30906 & $0.97 / 0.79$ & $52.9 / 47.1$ \\
\hline & 52994 & 28515 & 31363 & $0.97 / 0.79$ & $52.5 / 47.5$ \\
\hline sample 2 & 53364 & 27642 & 28943 & $0.97 / 0.79$ & $53.7 / 46.3$ \\
\hline & 55121 & 28645 & 29998 & $0.97 / 0.79$ & $53.7 / 46.3$ \\
\hline average & 53564 & 28327 & 30303 & $0.97 / 0.79$ & $53.2 / 46.8$ \\
\hline
\end{tabular}

\begin{tabular}{l|c|c|c|c|c|}
\cline { 2 - 6 } reaction 2 & area naphth & area 14 & area 15 & response factor 14/15 & ratio 14/15×100 \\
\hline sample 1 & 63211 & 34126 & 37476 & $0.97 / 0.79$ & $52.6 / 47.4$ \\
\hline & 62612 & 33889 & 37294 & $0.97 / 0.79$ & $52.5 / 47.5$ \\
\hline sample 2 & 62206 & 31979 & 33055 & $0.97 / 0.79$ & $54.0 / 46.0$ \\
\hline & 60815 & 31267 & 32562 & $0.97 / 0.79$ & $53.9 / 46.1$ \\
\hline average & 62211 & 32815 & 35097 & $0.97 / 0.79$ & $53.3 / 46.7$ \\
\hline
\end{tabular}


Competition of $(E)$-Dimethyl-(1-pentenyl)silanol (1) vs (E)-Di-(1-methylethyl)-(1 heptenyl)silanol (4) with 4-Iodoanisole

Following General Procedure II, 1 (28.8 mg, $0.20 \mathrm{mmol}), 4$ (45.6 mg, $0.20 \mathrm{mmol})$, 4iodoanisole $(46.8 \mathrm{mg}, 0.20 \mathrm{mmol})$, a solution of naphthalene $(0.8 \mathrm{~mL}, 0.20 \mathrm{mmol}, 0.25 \mathrm{M}$ in THF) and TBAF (0.8 mL, $0.80 \mathrm{mmol}, 1.0 \mathrm{M}$ in THF) and Pd(dba) 2 (5.8 $\mathrm{mg} 0.01 \mathrm{mmol})$ was stirred at room temperature for $1 \mathrm{~h}$ and then two sample aliquots were taken and analyzed twice on GC. GC analysis of samples showed a $\mathbf{1 4 / 1 5}$ ratio of 61.7/38.3.

GC Data:

\begin{tabular}{|c|c|c|c|c|c|}
\hline reaction 1 & area naphth & area 14 & area 15 & response factor $14 / 15$ & ratio $14 / 15 \times 100$ \\
\hline \multirow[t]{2}{*}{ sample 1} & 54347 & 26594 & 19788 & $0.97 / 0.79$ & $62.1 / 37.9$ \\
\hline & 53748 & 26239 & 19719 & $0.97 / 0.79$ & $61.8 / 38.2$ \\
\hline \multirow[t]{2}{*}{ sample 2} & 51248 & 25325 & 19255 & $0.97 / 0.79$ & $61.6 / 38.4$ \\
\hline & 55214 & 26984 & 20228 & $0.97 / 0.79$ & $61.9 / 38.1$ \\
\hline average & 53639 & 26286 & 19748 & $0.97 / 0.79$ & $61.9 / 38.1$ \\
\hline
\end{tabular}

\begin{tabular}{l|c|c|c|c|c|}
\cline { 2 - 6 } reaction 2 & area naphth & area 14 & area 15 & response factor 14/15 & ratio 14/15×100 \\
\hline sample 1 & 81797 & 47840 & 36408 & $0.97 / 0.79$ & $61.6 / 38.4$ \\
\hline & 72884 & 43003 & 32741 & $0.97 / 0.79$ & $61.6 / 38.4$ \\
\hline sample 2 & 75205 & 43959 & 33739 & $0.97 / 0.79$ & $61.3 / 38.7$ \\
\hline & 76017 & 45032 & 34276 & $0.97 / 0.79$ & $61.6 / 38.4$ \\
\hline average & 76476 & 44959 & 34291 & $0.97 / 0.79$ & $61.5 / 38.5$ \\
\hline
\end{tabular}

Competition of (E)-Dimethyl-(1-pentenyl)silanol (1) vs (E)-Di-(1,1-dimethylethyl)-(1 heptenyl)silanol (5) with 4-Iodoanisole

Following General Procedure II, 1 (28.8 mg, $0.20 \mathrm{mmol}), 5(51.2 \mathrm{mg}, 0.20 \mathrm{mmol})$, 4iodoanisole $(46.8 \mathrm{mg}, 0.20 \mathrm{mmol})$, a solution of naphthalene $(0.8 \mathrm{~mL}, 0.20 \mathrm{mmol}, 0.25 \mathrm{M}$ in THF) and TBAF (0.8 mL, $0.80 \mathrm{mmol}, 1.0 \mathrm{M}$ in THF) and $\mathrm{Pd}(\mathrm{dba})_{2}(5.8 \mathrm{mg} 0.01 \mathrm{mmol})$ was stirred at room temperature for $1 \mathrm{~h}$ and then two sample aliquots were taken and analyzed twice on GC. GC analysis of samples showed a 14/15 ratio of 96.1/3.9. 
GC Data:

\begin{tabular}{l|c|c|c|c|c|}
\cline { 2 - 6 } reaction 1 & area naphth & area 14 & area 15 & response factor 14/15 & ratio 14/15×100 \\
\hline sample 1 & 55450 & 47522 & 2288 & $0.97 / 0.79$ & $96.1 / 3.9$ \\
\hline & 54932 & 47437 & 2288 & $0.97 / 0.79$ & $96.2 / 3.8$ \\
\hline sample 2 & 54793 & 48162 & 2439 & $0.97 / 0.79$ & $96.0 / 4.0$ \\
\hline & 54829 & 48032 & 2455 & $0.97 / 0.79$ & $96.0 / 4.0$ \\
\hline average & 55001 & 47788 & 2368 & $0.97 / 0.79$ & $96.1 / 3.9$ \\
\hline
\end{tabular}

\begin{tabular}{l|c|c|c|c|c|}
\cline { 2 - 6 } reaction 2 & area naphth & area 14 & area 15 & response factor 14/15 & ratio 14/15×100 \\
\hline sample1 & 71951 & 62296 & 2900 & $0.97 / 0.79$ & $96.3 / 3.7$ \\
\hline & 72755 & 62632 & 2910 & $0.97 / 0.79$ & $96.3 / 3.7$ \\
\hline sample2 & 42415 & 37516 & 1926 & $0.97 / 0.79$ & $96.0 / 4.0$ \\
\hline & 43065 & 37744 & 1942 & $0.97 / 0.79$ & $95.9 / 4.1$ \\
\hline average & 57547 & 50047 & 2420 & $0.97 / 0.79$ & $96.1 / 3.9$ \\
\hline
\end{tabular}

\section{Competition of (E)-Dimethyl-(1-pentenyl)silanol (1) vs (E)-Diphenyl-(1-heptenyl)silanol (6) with 4-Iodoanisole}

Following General Procedure II, 1 (28.8 mg, $0.20 \mathrm{mmol}), 6$ (59.2 mg, $0.20 \mathrm{mmol})$, 4iodoanisole $(46.8 \mathrm{mg}, 0.20 \mathrm{mmol})$, a solution of naphthalene $(0.8 \mathrm{~mL}, 0.20 \mathrm{mmol}, 0.25 \mathrm{M}$ in THF) and TBAF (0.8 mL, $0.80 \mathrm{mmol}, 1.0 \mathrm{M}$ in THF) and $\mathrm{Pd}(\mathrm{dba})_{2}(5.8 \mathrm{mg} 0.01 \mathrm{mmol})$ was stirred at room temperature for $1 \mathrm{~h}$ and then two sample aliquots were taken and analyzed twice on GC. GC analysis of samples showed a $\mathbf{1 4 / 1 5}$ ratio of 56.3/43.7.

GC Data:

\begin{tabular}{|c|c|c|c|c|c|}
\hline reaction 1 & area naphth & area 14 & area 15 & response factor $14 / 15$ & ratio $14 / 15 \times 100$ \\
\hline \multirow[t]{2}{*}{ sample 1} & 32648 & 17120 & 16258 & $0.97 / 0.79$ & $56.2 / 43.8$ \\
\hline & 34431 & 17747 & 17204 & $0.97 / 0.79$ & $55.6 / 44.3$ \\
\hline \multirow[t]{2}{*}{ sample 2} & 37270 & 20655 & 19817 & $0.97 / 0.79$ & $55.9 / 44.1$ \\
\hline & 39301 & 21202 & 20540 & $0.97 / 0.79$ & $55.7 / 44.3$ \\
\hline average & 35913 & 19181 & 18455 & $0.97 / 0.79$ & $55.9 / 44.1$ \\
\hline
\end{tabular}




\begin{tabular}{l|c|c|c|c|c|}
\cline { 2 - 6 } reaction 2 & area naphth & area 14 & area 15 & response factor 14/15 & ratio 14/15×100 \\
\hline sample 1 & 40364 & 21219 & 19995 & $0.97 / 0.79$ & $56.3 / 43.6$ \\
\hline & 42465 & 21045 & 19731 & $0.97 / 0.79$ & $56.5 / 43.5$ \\
\hline sample 2 & 49337 & 24853 & 22506 & $0.97 / 0.79$ & $57.3 / 42.7$ \\
\hline & 46962 & 24173 & 22295 & $0.97 / 0.79$ & $56.9 / 43.1$ \\
\hline average & 44782 & 22823 & 21132 & $0.97 / 0.79$ & $56.8 / 43.2$ \\
\hline
\end{tabular}

\section{Competition of (E)-Dimethyl-(1-pentenyl)silanol (1) vs (E)-Trifluoropropyl-(1-heptenyl)- methylsilanol (7) with 4-Iodoanisole}

Following General Procedure V (see p 51), 1 (28.8 mg, $0.20 \mathrm{mmol}), 7(50.8 \mathrm{mg}, 0.20$ mmol), 4-iodotoluene ( $46.8 \mathrm{mg}, 0.20 \mathrm{mmol})$, a solution of naphthalene $(0.8 \mathrm{~mL}, 0.20 \mathrm{mmol}, 0.25$ $\mathrm{M}$ in THF) and TBAF (0.8 mL, $0.80 \mathrm{mmol}, 1.0 \mathrm{M}$ in THF) was stirred for $1 \mathrm{~h}$ and $\mathrm{Pd}(\mathrm{dba})_{2}(5.8$ $\mathrm{mg} 0.01 \mathrm{mmol}$ ) was added. The reaction was stirred at room temperature for $1 \mathrm{~h}$ and then two sample aliquots were taken and analyzed twice on GC. GC analysis of samples showed a 14/15 ratio of $44.6 / 55.4$.

GC Data:

\begin{tabular}{l|c|c|c|c|c|}
\cline { 2 - 6 } reaction 1 & area naphth & area 14 & area 15 & response factor 14/15 & ratio 14/15x100 \\
\hline sample 1 & 19529 & 7840 & 11073 & $0.96 / 0.79$ & $46.3 / 53.7$ \\
\hline & 19238 & 7758 & 11028 & $0.96 / 0.79$ & $46.2 / 53.8$ \\
\hline sample 2 & 15705 & 6999 & 11244 & $0.96 / 0.79$ & $43.1 / 56.9$ \\
\hline & 15460 & 6956 & 11174 & $0.96 / 0.79$ & $43.1 / 56.9$ \\
\hline average & 17483 & 7388 & 11130 & $0.96 / 0.79$ & $44.7 / 55.3$ \\
\hline
\end{tabular}

\begin{tabular}{l|c|c|c|c|c|}
\cline { 2 - 6 } reaction 2 & area naphth & area 14 & area 15 & response factor 14/15 & ratio 14/15x100 \\
\hline sample 1 & 14099 & 5661 & 8606 & $0.96 / 0.79$ & $44.5 / 55.5$ \\
\hline & 13931 & 6098 & 8592 & $0.96 / 0.79$ & $46.4 / 53.6$ \\
\hline sample 2 & 16252 & 7291 & 11442 & $0.96 / 0.79$ & $43.7 / 56.3$ \\
\hline & 14740 & 6575 & 10250 & $0.96 / 0.79$ & $43.8 / 56.2$ \\
\hline average & 14756 & 6406 & 9723 & $0.96 / 0.79$ & $44.6 / 55.4$ \\
\hline
\end{tabular}




\section{Competition Experiments with 2-Iodotoluene. General Procedure III.}
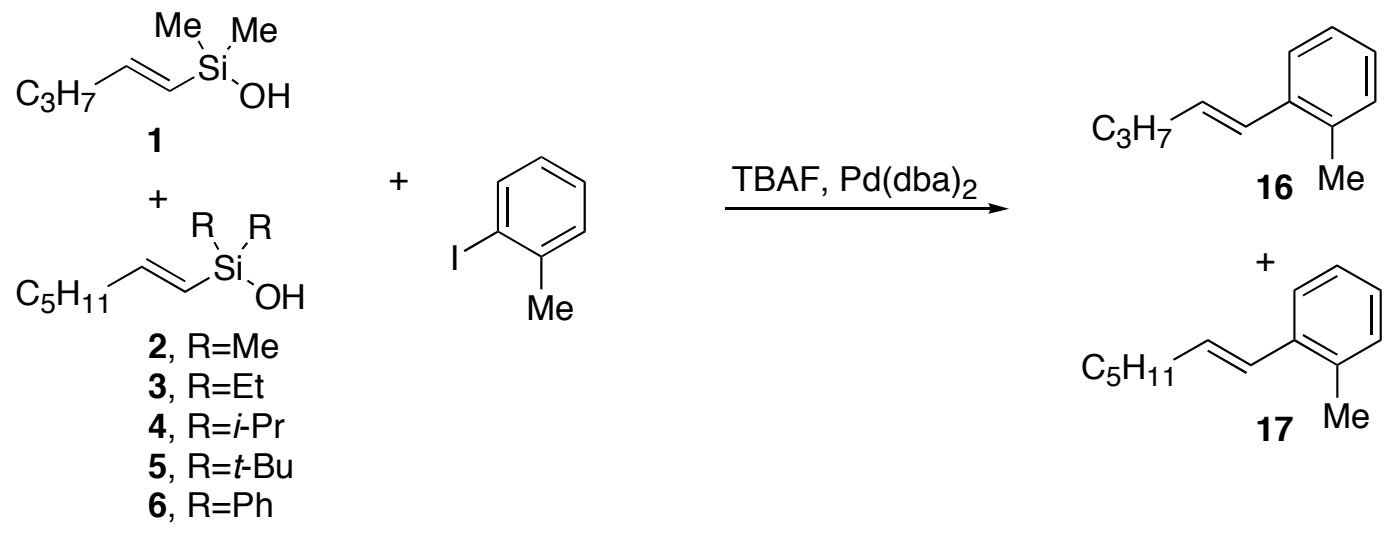

(E)-Dimethyl-(1-pentenyl)silanol (1), together with one of the other 1-heptenylsilanols (2-6), naphthalene, and $\mathrm{Pd}(\mathrm{dba})_{2}$ were dissolved in a TBAF solution (1.0 $\mathrm{M}$ in THF) in a flamedried, 5-mL, 2-neck, round-bottomed flask under $\mathrm{N}_{2}$. The 2-iodotoluene was then added slowly to maintain an internal temperature $<30{ }^{\circ} \mathrm{C}$. After $30 \mathrm{~min} 25-\mu \mathrm{L}$ samples were taken via syringe. The sample aliquots were filtered through a plug of silica gel washing with pentane to achieve a total sample volume of $\sim 2 \mathrm{~mL}$. These samples were then subjected to GC analysis. The reaction mixture was filtered through a plug of silica gel $(\sim 15 \mathrm{~g})$. The plug was washed with hexane/ethyl acetate, 9/1, (100 mL), and the solvent was evaporated in vacuo. The residue was purified by column chromatography (Reverse Phase $\mathrm{C} 18, \mathrm{MeOH} / \mathrm{H}_{2} \mathrm{O}, 9 / 1$ ) to afford the 16, and 17 which were further purified by Kugelrohr distillation.

\section{Competition of $(E)$-Dimethyl-(1-pentenyl)silanol (1) vs (E)-Dimethyl-(1-heptenyl)silanol (2)} with 2-Iodotoluene

Following General Procedure III, $1(86 \mu \mathrm{L}, 0.50 \mathrm{mmol}), 2(86.2 \mathrm{mg}, 0.50 \mathrm{mmol})$, naphthalene (65.1 mg, $0.51 \mathrm{mmol})$, TBAF (2.0 mL, $2.0 \mathrm{mmol}, 1.0 \mathrm{M}$ in THF), Pd(dba) $2(14.4$ mg $0.025 \mathrm{mmol})$, and 2-iodotoluene $(63.6 \mu \mathrm{L}, 0.50 \mathrm{mmol})$ was stirred at room temperature for $30 \mathrm{~min}$ and then sample aliquots were taken and reaction was worked up to afford $35 \mathrm{mg}$ (44\%) of $\mathbf{1 6}$ and $42 \mathrm{mg}$ (45\%) of 17. GC analysis of samples showed a 16/17 ratio of 49.4/50.6. 
GC Data:

\begin{tabular}{l|c|c|c|c|c|}
\cline { 2 - 6 } & area naphth & area 16 & area 17 & response factor 16/17 & ratio 16/17 100 \\
\hline sample 1 & 22228 & 13304 & 16519 & $0.85 / 0.71$ & $49.3 / 50.7$ \\
\hline sample 2 & 19390 & 11289 & 14030 & $0.85 / 0.71$ & $49.3 / 50.7$ \\
\hline sample 3 & 19452 & 11439 & 13917 & $0.85 / 0.71$ & $49.8 / 50.2$ \\
\hline average & 20357 & 12011 & 14822 & $0.85 / 0.71$ & $49.4 / 50.6$ \\
\hline
\end{tabular}

Competition of $(E)$-Dimethyl-(1-pentenyl)silanol (1) vs (E)-Dimethyl-(1-heptenyl)silanol (2) with 2-Iodotoluene

Following General Procedure III, $1(86 \mu \mathrm{L}, 0.50 \mathrm{mmol}), 2(86.2 \mathrm{mg}, 0.50 \mathrm{mmol})$, naphthalene (64.4 mg, $0.50 \mathrm{mmol})$, TBAF (2.0 mL, $2.0 \mathrm{mmol}, 1.0 \mathrm{M}$ in THF), Pd(dba)2 (14.4 mg $0.025 \mathrm{mmol})$, and 2-iodotoluene $(63.6 \mu \mathrm{L}, 0.50 \mathrm{mmol})$ was stirred at room temperature for $30 \mathrm{~min}$ and then sample aliquots were taken and reaction was worked up to afford $35 \mathrm{mg}(44 \%)$ of 16 and $42 \mathrm{mg} \mathrm{(45 \% )} \mathrm{of} \mathrm{17.} \mathrm{GC} \mathrm{analysis} \mathrm{of} \mathrm{samples} \mathrm{showed} \mathrm{a} \mathrm{16/17} \mathrm{ratio} \mathrm{of} \mathrm{50.9/49.1.}$ GC Data:

\begin{tabular}{l|c|c|c|c|c|}
\cline { 2 - 6 } & area naphth & area 16 & area 17 & response factor 16/17 & ratio 16/17×100 \\
\hline sample 1 & 7524 & 3507 & 4071 & $0.85 / 0.71$ & $50.9 / 49.1$ \\
\hline sample 2 & 7675 & 3501 & 4117 & $0.85 / 0.71$ & $50.7 / 49.3$ \\
\hline & 12969 & 5873 & 6829 & $0.85 / 0.71$ & $50.9 / 49.1$ \\
\hline sample 3 & 11107 & 5041 & 5868 & $0.85 / 0.71$ & $50.9 / 49.1$ \\
\hline & 10846 & 5052 & 5896 & $0.85 / 0.71$ & $50.9 / 49.1$ \\
\hline average & 10509 & 4810 & 5605 & $0.85 / 0.71$ & $50.9 / 49.1$ \\
\hline
\end{tabular}

\section{Competition of (E)-Dimethyl-(1-pentenyl)silanol (1) vs (E)-Diethyl-(1-heptenyl)silanol (3) with 2-Iodotoluene}

Following General Procedure II, $1(86 \mu \mathrm{L}, 0.50 \mathrm{mmol}), 3(100.6 \mathrm{mg}, 0.50 \mathrm{mmol})$, naphthalene (64.6 mg, $0.50 \mathrm{mmol})$, TBAF (2.0 mL, $2.0 \mathrm{mmol}, 1.0 \mathrm{M}$ in THF), Pd(dba) $2(14.4$ $\mathrm{mg} 0.025 \mathrm{mmol})$, and 2-iodotoluene $(63.6 \mu \mathrm{L}, 0.50 \mathrm{mmol})$ was stirred at room temperature for $30 \mathrm{~min}$ and then sample aliquots were taken and reaction was worked up to afford $42 \mathrm{mg}(52 \%)$ of 16 and $39 \mathrm{mg}$ (42\%) of 17. GC analysis of samples showed a 16/17 ratio of 57.5/42.5. 
GC Data:

\begin{tabular}{l|c|c|c|c|c|}
\cline { 2 - 6 } & area naphth & area 16 & area 17 & response factor 16/17 & ratio 16/17×100 \\
\hline sample 1 & 19186 & 14700 & 13093 & $0.85 / 0.71$ & $57.5 / 42.5$ \\
\hline sample 2 & 11585 & 8532 & 7937 & $0.85 / 0.71$ & $56.5 / 43.5$ \\
\hline sample 3 & 9163 & 6769 & 5760 & $0.85 / 0.71$ & $58.6 / 41.4$ \\
\hline average & 13311 & 10000 & 8930 & $0.85 / 0.71$ & $57.5 / 42.5$ \\
\hline
\end{tabular}

Competition of (E)-Dimethyl-(1-pentenyl)silanol (1) vs (E)-Diethyl-(1-heptenyl)silanol (3) with 2-Iodotoluene

Following General Procedure III, 1 (86 $\mu \mathrm{L}, 0.50 \mathrm{mmol}), 3$ (100.1 mg, $0.50 \mathrm{mmol})$, naphthalene (66.2 mg, $0.52 \mathrm{mmol})$, TBAF (2.0 mL, $2.0 \mathrm{mmol}, 1.0 \mathrm{M}$ in THF), Pd(dba)2 (14.4 mg $0.025 \mathrm{mmol})$, and 2-iodotoluene $(63.6 \mu \mathrm{L}, 0.50 \mathrm{mmol})$ was stirred at room temperature for $30 \mathrm{~min}$ and then sample aliquots were taken and reaction was worked up to afford $41 \mathrm{mg}(51 \%)$ of 16 and $33 \mathrm{mg}$ (35\%) of 17. GC analysis of samples showed a 16/17 ratio of 59.6/40.4. GC Data:

\begin{tabular}{l|c|c|c|c|c|}
\cline { 2 - 6 } & area naphth & area 16 & area 17 & response factor 16/17 & ratio 16/17×100 \\
\hline sample 1 & 12814 & 7894 & 6425 & $0.85 / 0.71$ & $59.7 / 40.3$ \\
\hline sample 2 & 12655 & 7761 & 6326 & $0.85 / 0.71$ & $59.7 / 40.3$ \\
\hline & 11794 & 7148 & 5773 & $0.85 / 0.71$ & $59.9 / 40.1$ \\
\hline sample 3 & 11860 & 7216 & 5768 & $0.85 / 0.71$ & $60.1 / 39.9$ \\
\hline & 10639 & 6591 & 5438 & $0.85 / 0.71$ & $58.9 / 41.1$ \\
\hline average & 11772 & 7190 & 5873 & $0.85 / 0.71$ & $59.4 / 40.6$ \\
\hline
\end{tabular}

\section{Competition of $(E)$-Dimethyl-(1-pentenyl)silanol (1) vs (E)-Di-(1-methylethyl)-(1-heptenyl)- silanol (4) with 2-Iodotoluene}

Following General Procedure III, 1 (86 $\mu \mathrm{L}, 0.50 \mathrm{mmol}), 4$ (114.7 mg, $0.50 \mathrm{mmol})$, naphthalene (64.2 mg, $0.50 \mathrm{mmol})$, TBAF (2.0 mL, $2.0 \mathrm{mmol}, 1.0 \mathrm{M}$ in THF), Pd(dba) $2(14.4$ $\mathrm{mg} 0.025 \mathrm{mmol})$, and 2-iodotoluene $(63.6 \mu \mathrm{L}, 0.50 \mathrm{mmol})$ was stirred at room temperature for $30 \mathrm{~min}$ and then sample aliquots were taken and reaction was worked up to afford $42 \mathrm{mg}(53 \%)$ of $\mathbf{1 6}$ and $36 \mathrm{mg}$ (38\%) of 17. GC analysis of samples showed a 16/17 ratio of 59.8/40.2. 
GC Data:

\begin{tabular}{l|c|c|c|c|c|}
\cline { 2 - 6 } & area naphth & area 16 & area 17 & response factor 16/17 & ratio 16/17×100 \\
\hline sample 1 & 74249 & 52466 & 41686 & $0.85 / 0.71$ & $60.3 / 39.7$ \\
\hline sample 2 & 74750 & 52972 & 42788 & $0.85 / 0.71$ & $59.9 / 40.1$ \\
\hline & 23057 & 16155 & 13050 & $0.85 / 0.71$ & $59.9 / 40.1$ \\
\hline sample 3 & 23271 & 16273 & 13294 & $0.85 / 0.71$ & $59.6 / 40.4$ \\
\hline & 27105 & 18791 & 15527 & $0.85 / 0.71$ & $59.4 / 40.6$ \\
\hline average & 41592 & 29232 & 23587 & $0.85 / 0.71$ & $59.8 / 40.2$ \\
\hline
\end{tabular}

Competition of (E)-Dimethyl-(1-pentenyl)silanol (1) vs (E)-Di(1-methylethyl)-(1-heptenyl)silanol (4) with 2-Iodotoluene

Following General Procedure III, $1(86 \mu \mathrm{L}, 0.50 \mathrm{mmol}), 4(114.7 \mathrm{mg}, 0.50 \mathrm{mmol})$, naphthalene (66.7 mg, $0.52 \mathrm{mmol}$ ), TBAF (2.0 mL, $2.0 \mathrm{mmol}, 1.0 \mathrm{M}$ in THF), Pd(dba) 2 (14.4 $\mathrm{mg} 0.025 \mathrm{mmol})$, and 2-iodotoluene $(63.6 \mu \mathrm{L}, 0.50 \mathrm{mmol})$ was stirred at room temperature for $30 \mathrm{~min}$ and then sample aliquots were taken and reaction was worked up to afford $43 \mathrm{mg}$ (54\%) of $\mathbf{1 6}$ and $32 \mathrm{mg}$ (34\%) of $\mathbf{1 7}$. GC analysis of samples showed a $\mathbf{1 6 / 1 7}$ ratio of 61.1/38.9. GC Data:

\begin{tabular}{l|c|c|c|c|c|}
\cline { 2 - 6 } & area naphth & area 16 & area 17 & response factor 16/17 & ratio 16/17×100 \\
\hline sample 1 & 9060 & 5548 & 4243 & $0.85 / 0.71$ & $61.2 / 38.8$ \\
\hline & 9143 & 5568 & 4247 & $0.85 / 0.71$ & $61.3 / 38 / 7$ \\
\hline sample 2 & 9447 & 5773 & 4473 & $0.85 / 0.71$ & $60.9 / 39.1$ \\
\hline sample 3 & 9477 & 5797 & 4453 & $0.85 / 0.71$ & $61.1 / 38.9$ \\
\hline & 12196 & 7549 & 5773 & $0.85 / 0.71$ & $61.2 / 38.8$ \\
\hline average & 12295 & 7597 & 5819 & $0.85 / 0.71$ & $61.2 / 38.8$ \\
\hline
\end{tabular}

\section{Competition of (E)-Dimethyl-(1-pentenyl)silanol (1) vs (E)-Di-(1,1-dimethylethyl)-(1- heptenyl)silanol (5) with 2-Iodotoluene}

Following General Procedure III, 1 ( $86 \mu \mathrm{L}, 0.50 \mathrm{mmol}), 5(129.7 \mathrm{mg}, 0.51 \mathrm{mmol})$, naphthalene (64.8 mg, $0.51 \mathrm{mmol})$, TBAF (2.0 mL, $2.0 \mathrm{mmol}, 1.0 \mathrm{M}$ in THF), Pd(dba) 2 (14.4 $\mathrm{mg} 0.025 \mathrm{mmol})$, and 2-iodotoluene $(63.6 \mu \mathrm{L}, 0.50 \mathrm{mmol})$ was stirred at room temperature for 
$30 \mathrm{~min}$ and then sample aliquots were taken and reaction was worked up to afford $67 \mathrm{mg}(84 \%)$ of 16. GC analysis of samples showed a $\mathbf{1 6 / 1 7}$ ratio of 100.0/0.0.

GC Data:

\begin{tabular}{l|c|c|c|c|c|}
\cline { 2 - 6 } & area naphth & area 16 & area 17 & response factor 16/17 & ratio 16/17×100 \\
\hline sample 1 & 10039 & 9771 & 0 & $0.85 / 0.71$ & $100 / 0$ \\
\hline sample 2 & 9995 & 9802 & 0 & $0.85 / 0.71$ & $100 / 0$ \\
\hline & 7633 & 7435 & 0 & $0.85 / 0.71$ & $100 / 0$ \\
\hline sample 3 & 8640 & 8404 & 0 & $0.85 / 0.71$ & $100 / 0$ \\
\hline & 8633 & 8453 & 0 & $0.85 / 0.71$ & $100 / 0$ \\
\hline average & 8764 & 8536 & 0 & $0.85 / 0.71$ & $100 / 0$ \\
\hline
\end{tabular}

\section{Competition of (E)-Dimethyl-(1-pentenyl)silanol (1) vs (E)-Di-(1,1-dimethylethyl)-(1-} heptenyl)silanol (5) with 2-Iodotoluene

Following General Procedure III, 1 (86 $\mu \mathrm{L}, 0.50 \mathrm{mmol}), 5(128.2 \mathrm{mg}, 0.50 \mathrm{mmol})$, naphthalene $(65.2 \mathrm{mg}, 0.51 \mathrm{mmol}), \mathrm{TBAF}(2.0 \mathrm{~mL}, 2.0 \mathrm{mmol}, 1.0 \mathrm{M}$ in THF), Pd(dba)2 (14.4 $\mathrm{mg} 0.025 \mathrm{mmol})$, and 2-iodotoluene $(63.6 \mu \mathrm{L}, 0.50 \mathrm{mmol})$ was stirred at room temperature for $30 \mathrm{~min}$ and then sample aliquots were taken and reaction was worked up to afford $65 \mathrm{mg}(82 \%)$ of 16. GC analysis of samples showed a 16/17 ratio of 100.0/0.0.

GC Data:

\begin{tabular}{l|c|c|c|c|c|}
\cline { 2 - 6 } & area naphth & area 16 & area 17 & response factor 16/17 & ratio 16/17x100 \\
\hline sample 1 & 8955 & 7390 & 0 & $0.85 / 0.71$ & $100 / 0$ \\
\hline sample 2 & 11055 & 9174 & 0 & $0.85 / 0.71$ & $100 / 0$ \\
\hline & 11114 & 9165 & 0 & $0.85 / 0.71$ & $100 / 0$ \\
\hline sample 3 & 10557 & 8605 & 0 & $0.85 / 0.71$ & $100 / 0$ \\
\hline & 10412 & 8650 & 0 & $0.85 / 0.71$ & $100 / 0$ \\
\hline average & 10176 & 8397 & 0 & $0.85 / 0.71$ & $100 / 0$ \\
\hline
\end{tabular}


Competition of $(E)$-Dimethyl-(1-pentenyl)silanol (1) vs (E)-Diphenyl-(1-heptenyl)silanol (6) with 2-Iodotoluene

Following General Procedure III, 1 (86 $\mu \mathrm{L}, 0.50 \mathrm{mmol}), 6$ (146.8 mg, $0.50 \mathrm{mmol})$, naphthalene (69.7 mg, $0.54 \mathrm{mmol})$, TBAF (2.0 mL, $2.0 \mathrm{mmol}, 1.0 \mathrm{M}$ in THF), Pd(dba)2 (14.4 mg $0.025 \mathrm{mmol})$, and 2-iodotoluene $(63.6 \mu \mathrm{L}, 0.50 \mathrm{mmol})$ was stirred at room temperature for $30 \mathrm{~min}$ and then sample aliquots were taken and reaction was worked up to afford $35 \mathrm{mg}(44 \%)$ of $\mathbf{1 6}$ and $42 \mathrm{mg}(45 \%)$ of $\mathbf{1 7}$. GC analysis of samples showed a 16/17 ratio of 50.1/49.9.

GC Data:

\begin{tabular}{|c|c|c|c|c|c|}
\hline & area naphth & area 16 & area 17 & response factor $16 / 17$ & ratio $16 / 17 \times 100$ \\
\hline \multirow[t]{2}{*}{ sample 1} & 18494 & 9737 & 11707 & $0.85 / 0.71$ & $50.1 / 49.9$ \\
\hline & 19365 & 10020 & 21041 & $0.85 / 0.71$ & $50.1 / 49.9$ \\
\hline \multirow[t]{2}{*}{ sample 2} & 19794 & 10345 & 12443 & $0.85 / 0.71$ & $50.1 / 49.9$ \\
\hline & 20137 & 10600 & 12710 & $0.85 / 0.71$ & $50.1 / 49.9$ \\
\hline \multirow[t]{2}{*}{ sample 3} & 15928 & 8320 & 10035 & $0.85 / 0.71$ & $50.5 / 50.0$ \\
\hline & 16400 & 8516 & 10157 & $0.85 / 0.71$ & $50.3 / 49.7$ \\
\hline average & 18353 & 9590 & 13016 & $0.85 / 0.71$ & $50.1 / 49.9$ \\
\hline
\end{tabular}

\section{Competition of (E)-Dimethyl-(1-pentenyl)silanol (1) vs (E)-Diphenyl-(1-heptenyl)silanol (6) with 2-Iodotoluene}

Following General Procedure III, 1 (86 $\mu \mathrm{L}, 0.50 \mathrm{mmol}), 6$ (149.3 mg, $0.50 \mathrm{mmol})$, naphthalene (70.3 mg, $0.55 \mathrm{mmol})$, TBAF (2.0 mL, $2.0 \mathrm{mmol}, 1.0 \mathrm{M}$ in THF), Pd(dba)2 (14.4 $\mathrm{mg} 0.025 \mathrm{mmol})$, and 2-iodotoluene $(63.6 \mu \mathrm{L}, 0.50 \mathrm{mmol})$ was stirred at room temperature for $30 \mathrm{~min}$ and then sample aliquots were taken and reaction was worked up to afford $36 \mathrm{mg}$ (45\%) of 16 and $43 \mathrm{mg}(45 \%)$ of $\mathbf{1 7}$. GC analysis of samples showed a 16/17 ratio of 50.2/49.8. 
GC Data:

\begin{tabular}{|c|c|c|c|c|c|}
\hline & area naphth & area 16 & area 17 & response factor $16 / 17$ & ratio $16 / 17 \times 100$ \\
\hline \multirow[t]{2}{*}{ sample 1} & 13621 & 7254 & 8332 & $0.85 / 0.71$ & $51.2 / 48.8$ \\
\hline & 14192 & 7372 & 8437 & $0.85 / 0.71$ & $51.3 / 48.7$ \\
\hline \multirow[t]{2}{*}{ sample 2} & 16065 & 8346 & 9522 & $0.85 / 0.71$ & $51.4 / 48.6$ \\
\hline & 16195 & 8446 & 9711 & $0.85 / 0.71$ & $51.2 / 48.8$ \\
\hline \multirow[t]{2}{*}{ sample 3} & 13461 & 6945 & 8040 & $0.85 / 0.71$ & $51.0 / 49.0$ \\
\hline & 13655 & 7067 & 8222 & $0.85 / 0.71$ & $50.9 / 49.1$ \\
\hline average & 14532 & 7572 & 8711 & $0.85 / 0.71$ & $50.2 / 49.8$ \\
\hline
\end{tabular}

Competition Experiment from Table 2 (Heteroatom Substituent Effects).

\section{Competition Experiments with 4-Iodoacetophenone. General Procedure IV.}<smiles>[R7][Si]([R7])([R7])/C=C/[CH+]</smiles>

1, $\mathrm{R}_{1}=\mathrm{R}_{2}=\mathrm{Me}$, $\mathrm{R}=\mathrm{H}$,

8, $\mathrm{R}_{1}=\mathrm{Me}$, $\mathrm{R}_{2}=\mathrm{OEt}, \mathrm{R}=\mathrm{Et} \quad+$ $R_{3}, R_{4}$<smiles>[R5][SiH2]C=C[AsH2-]</smiles>

$10, \mathrm{R}_{3}=\mathrm{Me}$, $\mathrm{R}_{4}=\mathrm{R}_{5}=\mathrm{OEt}$,

11, $\mathrm{R}_{3}=\mathrm{R}_{4}=\mathrm{OEt}$, $\mathrm{R}_{5}=\mathrm{OEt}$
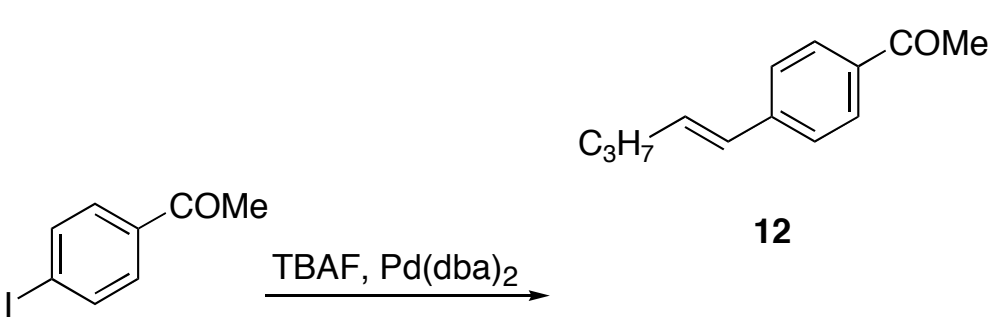

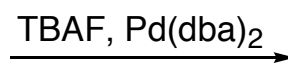<smiles>CC(=O)c1ccc(/C=C/[SbH2])cc1</smiles>

13

A flame-dried, 5-mL, 2-neck, round-bottomed flask under $\mathrm{N}_{2}$ was charged with $(E)$ dimethyl-(1-pentenyl)silanol (1) or (E)-diethoxy-(1-pentenyl)methylsilane (8), $(E)$-triethoxy-(1heptenyl)silane (10) or (E)-diethoxy-(1-heptenyl)methylsilane (11), and 4-iodoacetophenone. THF solutions of naphthalene $(0.25 \mathrm{M})$ and TBAF $(1.0 \mathrm{M})$ were added next. The mixture was stirred at room temperature for $1 \mathrm{~h}$ and $\mathrm{Pd}(\mathrm{dba})_{2}$ was added. After 30 min two $25-\mu \mathrm{L}$ samples were taken via syringe. The sample aliquots were filtered through a plug of silica gel washing with $\mathrm{Et}_{2} \mathrm{O}$ to achieve a total sample volume of $\sim 2 \mathrm{~mL}$. These samples were then subjected to GC analysis. Reactions were performed in duplicate. 
Competition of $(E)$-Dimethyl-(1-pentenyl)silanol (1) vs (E)-Diethoxy-(1-heptenyl)methylsilane (10) with 4-Iodoacetophenone

Following General Procedure IV, 1 (28.8 mg, $0.20 \mathrm{mmol}), 15$ (46.0 mg, $0.20 \mathrm{mmol})$, 4iodoacetophenone $(49.2 \mathrm{mg}, 0.20 \mathrm{mmol})$, a solution of naphthalene $(0.8 \mathrm{~mL}, 0.20 \mathrm{mmol}, 0.25 \mathrm{M}$ in THF) and TBAF (0.8 mL, $0.80 \mathrm{mmol}, 1.0 \mathrm{M}$ in THF) was stirred for $1 \mathrm{~h}$ and $\mathrm{Pd}(\mathrm{dba})_{2}(5.8 \mathrm{mg}$ $0.01 \mathrm{mmol}$ ) was added. The reaction was stirred at room temperature for $1 \mathrm{~h}$ and then two sample aliquots were taken and analyzed twice on GC. GC analysis of samples showed a 12/13 ratio of $49.2 / 50.8$.

GC Data:

\begin{tabular}{l|c|c|c|c|c|}
\cline { 2 - 6 } reaction 1 & area naphth & area 12 & area 13 & response factor 12/13 & ratio 12/13×100 \\
\hline sample 1 & 43234 & 22649 & 28321 & $0.78 / 0.67$ & $48.4 / 51.6$ \\
\hline & 43001 & 20833 & 26167 & $0.78 / 0.67$ & $48.3 / 51.7$ \\
\hline sample 2 & 24316 & 15081 & 18853 & $0.78 / 0.67$ & $48.4 / 51.6$ \\
\hline & 31582 & 15703 & 18656 & $0.78 / 0.67$ & $49.7 / 50.3$ \\
\hline average & 35533 & 18567 & 22999 & $0.78 / 0.67$ & $48.7 / 51.3$ \\
\hline
\end{tabular}

\begin{tabular}{l|c|c|c|c|c|}
\cline { 2 - 6 } reaction 2 & area naphth & area 12 & area 13 & response factor 12/13 & ratio 12/13×100 \\
\hline sample 1 & 33537 & 16512 & 18458 & $0.78 / 0.67$ & $51.2 / 48.8$ \\
\hline & 37849 & 18562 & 21433 & $0.78 / 0.67$ & $50.4 / 49.6$ \\
\hline sample 2 & 36630 & 16186 & 20262 & $0.78 / 0.67$ & $48.4 / 51.6$ \\
\hline & 44103 & 19495 & 23721 & $0.78 / 0.67$ & $49.0 / 51.0$ \\
\hline average & 38030 & 17689 & 20969 & $0.78 / 0.67$ & $49.8 / 50.2$ \\
\hline
\end{tabular}

Competition of (E)-Dimethyl-(1-pentenyl)silanol (1) vs (E)-Triethoxy-(1-heptenyl)silane (11) with 4-Iodoacetophenone

Following General Procedure IV, 1 (28.8 mg, 0.20 mmol), 11 (52 mg, 0.20 mmol), 4iodoacetophenone $(49.2 \mathrm{mg}, 0.20 \mathrm{mmol})$, a solution of naphthalene $(0.8 \mathrm{~mL}, 0.20 \mathrm{mmol}, 0.25 \mathrm{M}$ in THF) and TBAF (0.8 mL, $0.80 \mathrm{mmol}, 1.0 \mathrm{M}$ in THF) was stirred for $1 \mathrm{~h}$ and $\mathrm{Pd}(\mathrm{dba})_{2}(5.8 \mathrm{mg}$ $0.01 \mathrm{mmol}$ ) was added. The reaction was stirred at room temperature for $1 \mathrm{~h}$ and then two sample aliquots were taken and analyzed twice on GC. GC analysis of samples showed a 12/13 ratio of $73.9 / 26.1$. 
GC Data:

\begin{tabular}{l|c|c|c|c|c|}
\cline { 2 - 6 } reaction 1 & area naphth & area 12 & area 13 & response factor 12/13 & ratio 12/13×100 \\
\hline sample 1 & 33285 & 19312 & 7574 & $0.78 / 0.67$ & $74.9 / 25.1$ \\
\hline & 34349 & 20642 & 8491 & $0.78 / 0.67$ & $74.0 / 26.0$ \\
\hline sample 2 & 41452 & 24629 & 10241 & $0.78 / 0.67$ & $73.8 / 26.2$ \\
\hline average & 37199 & 23529 & 9387 & $0.78 / 0.67$ & $74.6 / 25.4$ \\
\hline
\end{tabular}

\begin{tabular}{l|c|c|c|c|c|}
\cline { 2 - 6 } reaction 2 & area naphth & area 12 & area 13 & response factor 12/13 & ratio 12/13x100 \\
\hline sample 1 & 31830 & 23506 & 9484 & $0.78 / 0.67$ & $74.4 / 25.6$ \\
\hline & 30942 & 22643 & 9222 & $0.78 / 0.67$ & $74.2 / 25.8$ \\
\hline sample 2 & 36959 & 23815 & 11330 & $0.78 / 0.67$ & $71.1 / 28.8$ \\
\hline & 34267 & 21527 & 8693 & $0.78 / 0.67$ & $74.4 / 25.6$ \\
\hline average & 33500 & 22873 & 9682 & $0.78 / 0.67$ & $73.5 / 26.5$ \\
\hline
\end{tabular}

\section{Competition of $(E)$ - Diethoxy-(1-pentenyl)methylsilane (8) vs (E)-Triethoxy-(1-heptenyl)- silane (11) with 4-Iodoacetophenone}

Following General Procedure IV, 8 (40.4 mg, $0.20 \mathrm{mmol}), 14$ (52.0 mg, $0.20 \mathrm{mmol})$, 4iodoacetophenone $(49.2 \mathrm{mg}, 0.20 \mathrm{mmol})$, a solution of naphthalene $(0.8 \mathrm{~mL}, 0.20 \mathrm{mmol}, 0.25 \mathrm{M}$ in THF) and TBAF (0.8 mL, $0.80 \mathrm{mmol}, 1.0 \mathrm{M}$ in THF) was stirred for $1 \mathrm{~h}$ and $\mathrm{Pd}(\mathrm{dba})_{2}(5.8 \mathrm{mg}$ $0.01 \mathrm{mmol}$ ) was added. The reaction was stirred at room temperature for $1 \mathrm{~h}$ and then two sample aliquots were taken and analyzed twice on GC. GC analysis of samples showed a 12/13 ratio of 73.1/26.9.

GC Data:

\begin{tabular}{|c|c|c|c|c|c|}
\hline reaction 1 & area naphth & area 12 & area 13 & response factor $12 / 13$ & ratio $12 / 13 \times 100$ \\
\hline \multirow[t]{2}{*}{ sample 1} & 46263 & 19698 & 8310 & $0.78 / 0.67$ & $73.5 / 26.5$ \\
\hline & 46379 & 21699 & 9886 & $0.78 / 0.67$ & $72.0 / 28.0$ \\
\hline \multirow[t]{2}{*}{ sample 2} & 39019 & 18565 & 8469 & $0.78 / 0.67$ & $72.0 / 28.0$ \\
\hline & 37506 & 18054 & 8248 & $0.78 / 0.67$ & $72.0 / 28.0$ \\
\hline average & 42292 & 19504 & 8728 & $0.78 / 0.67$ & $72.4 / 27.6$ \\
\hline
\end{tabular}




\begin{tabular}{|c|c|c|c|c|c|}
\hline reaction 2 & area naphth & area 12 & area 13 & response factor $12 / 13$ & ratio $12 / 13 \times 100$ \\
\hline \multirow[t]{2}{*}{ sample 1} & 34306 & 20132 & 8667 & $0.78 / 0.67$ & $73.1 / 26.9$ \\
\hline & 34281 & 20059 & 8871 & $0.78 / 0.67$ & $72.6 / 27.4$ \\
\hline \multirow[t]{2}{*}{ sample 2} & 29777 & 17456 & 6818 & $0.78 / 0.67$ & $75.0 / 25.0$ \\
\hline & 29877 & 17692 & 6950 & $0.78 / 0.67$ & $74.9 / 25.1$ \\
\hline average & 32060 & 18835 & 7827 & $0.78 / 0.67$ & $73.9 / 26.1$ \\
\hline
\end{tabular}

\section{Competition Experiments with 4-Iodoanisole. General Procedure V.}

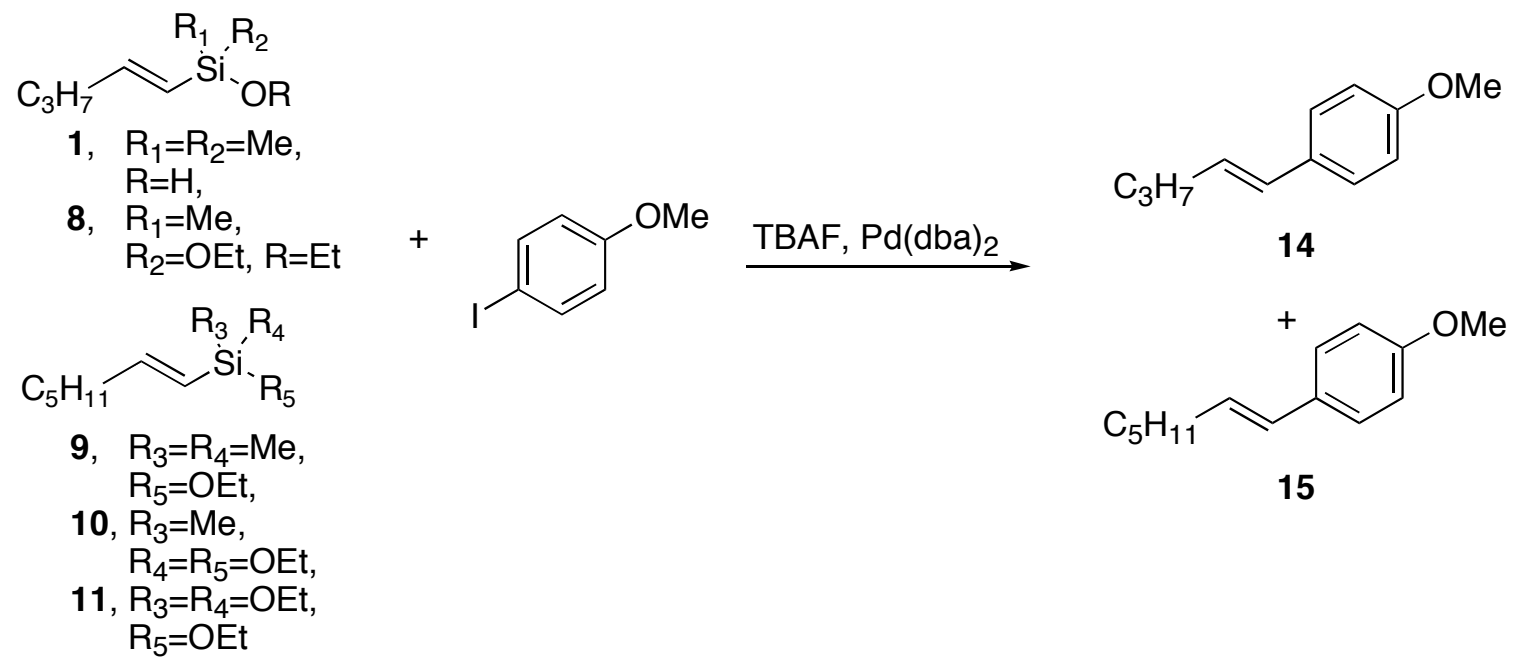

A flame-dried, 5-mL, 2-neck, round-bottomed flask under $\mathrm{N}_{2}$ was charged with $(E)$ dimethyl-(1-pentenyl)silanol (1) or $(E)$-diethoxy-(1-pentenyl)methylsilane $(\mathbf{8}),(E)$-diethoxy-(1heptenyl)methylsilane (11) or (E)-Triethoxy-(1-heptenyl)silane (15) or (E)-dimethylethoxy-(1heptenyl)silane (17) and 4-iodoanisole. THF solutions of naphthalene (0.25 M) and TBAF (1.0 M) were added next. The mixture was stirred at room temperature for $1 \mathrm{~h}$ and $\operatorname{Pd}(\mathrm{dba})_{2}$ was added. After 30 min two $25-\mu \mathrm{L}$ samples were taken via syringe. The sample aliquots were filtered through a plug of silica gel washing with $\mathrm{Et}_{2} \mathrm{O}$ to achieve a total sample volume of $\sim 2$ $\mathrm{mL}$. These samples were then subjected to GC analysis. Reactions were performed in duplicate.

\section{Competition of (E)-Dimethyl-(1-pentenyl)silanol (1) vs (E)-Diethoxy-(1-heptenyl)methyl- silane (10) with 4-Iodoanisole}

Following General Procedure V, 1 (28.8 mg, 0.20 mmol), 10 (46.0 mg, 0.20 mmol), 4iodoanisole $(46.8 \mathrm{mg}, 0.20 \mathrm{mmol})$, a solution of naphthalene $(0.8 \mathrm{~mL}, 0.20 \mathrm{mmol}, 0.25 \mathrm{M}$ in 
THF) and TBAF (0.8 mL, $0.80 \mathrm{mmol}, 1.0 \mathrm{M}$ in THF) was stirred for $1 \mathrm{~h}$ and $\mathrm{Pd}(\mathrm{dba})_{2}(5.8 \mathrm{mg}$ $0.01 \mathrm{mmol}$ ) was added. The reaction was stirred at room temperature for $1 \mathrm{~h}$ and then two sample aliquots were taken and analyzed twice on GC. GC analysis of samples showed a 14/15 ratio of 56.6/43.4.

GC Data:

\begin{tabular}{l|c|c|c|c|c|}
\cline { 2 - 6 } reaction 1 & area naphth & area 14 & area 15 & response factor 14/15 & ratio 14/15x100 \\
\hline sample 1 & 33508 & 19836 & 19364 & $0.96 / 0.79$ & $55.5 / 44.5$ \\
\hline & 35651 & 20941 & 19949 & $0.96 / 0.79$ & $56.1 / 43.9$ \\
\hline sample 2 & 39588 & 24024 & 21623 & $0.96 / 0.79$ & $57.5 / 42.5$ \\
\hline & 40440 & 24611 & 22066 & $0.96 / 0.79$ & $57.6 / 42.4$ \\
\hline average & 37297 & 22353 & 20751 & $0.96 / 0.79$ & $56.7 / 43.3$ \\
\hline
\end{tabular}

\begin{tabular}{l|c|c|c|c|c|}
\cline { 2 - 6 } reaction 2 & area naphth & area 14 & area 15 & response factor 14/15 & ratio 14/15x100 \\
\hline sample 1 & 36310 & 21747 & 19973 & $0.96 / 0.79$ & $57.0 / 43.0$ \\
\hline & 35890 & 21662 & 19462 & $0.96 / 0.79$ & $57.5 / 42.5$ \\
\hline sample 2 & 59786 & 35099 & 33646 & $0.96 / 0.79$ & $56.0 / 44.0$ \\
\hline & 59901 & 35132 & 33666 & $0.96 / 0.79$ & $56.0 / 44.0$ \\
\hline average & 47972 & 28410 & 26687 & $0.96 / 0.79$ & $56.6 / 43.4$ \\
\hline
\end{tabular}

\section{Competition of (E)-Dimethyl-(1-pentenyl)silanol (1) vs (E)-Triethoxy-(1-heptenyl)silane}

\section{(11) with 4-Iodoanisole}

Following General Procedure V, 1 (28.8 mg, $0.20 \mathrm{mmol}), 11$ (52.0 mg, $0.20 \mathrm{mmol})$, 4iodoanisole $(46.8 \mathrm{mg}, 0.20 \mathrm{mmol})$, a solution of naphthalene $(0.8 \mathrm{~mL}, 0.20 \mathrm{mmol}, 0.25 \mathrm{M}$ in THF) and TBAF (0.8 mL, $0.80 \mathrm{mmol}, 1.0 \mathrm{M}$ in THF) was stirred for $1 \mathrm{~h}$ and $\mathrm{Pd}(\mathrm{dba})_{2}(5.8 \mathrm{mg}$ $0.01 \mathrm{mmol}$ ) was added. The reaction was stirred at room temperature for $1 \mathrm{~h}$ and then two sample aliquots were taken and analyzed twice on GC. GC analysis of samples showed a 14/15 ratio of $81.5 / 18.5$. 
GC Data:

\begin{tabular}{l|c|c|c|c|c|}
\cline { 2 - 6 } reaction 1 & area naphth & area 14 & area 15 & response factor 14/15 & ratio 14/15×100 \\
\hline sample 1 & 63425 & 43224 & 11789 & $0.96 / 0.79$ & $81.7 / 18.3$ \\
\hline & 63784 & 43594 & 11822 & $0.96 / 0.79$ & $81.8 / 18.2$ \\
\hline sample 2 & 39579 & 27782 & 7613 & $0.96 / 0.79$ & $81.6 / 18.4$ \\
\hline & 40022 & 28112 & 7527 & $0.96 / 0.79$ & $81.9 / 18.1$ \\
\hline average & 51703 & 35678 & 9688 & $0.96 / 0.79$ & $81.8 / 18.2$ \\
\hline
\end{tabular}

\begin{tabular}{l|c|c|c|c|c|}
\cline { 2 - 6 } reaction 2 & area naphth & area 14 & area 15 & response factor 14/15 & ratio 14/15×100 \\
\hline sample 1 & 62306 & 48939 & 13656 & $0.96 / 0.79$ & $81.4 / 18.6$ \\
\hline & 63805 & 49961 & 13845 & $0.96 / 0.79$ & $81.5 / 18.5$ \\
\hline sample 2 & 58500 & 46520 & 12973 & $0.96 / 0.79$ & $81.4 / 18.6$ \\
\hline & 58898 & 46305 & 12986 & $0.96 / 0.79$ & $81.3 / 18.7$ \\
\hline average & 60877 & 47931 & 13365 & $0.96 / 0.79$ & $81.4 / 18.6$ \\
\hline
\end{tabular}

\section{Competition of $(E)$ - Diethoxy-(1-pentenyl)methylsilane (8) vs (E)-Triethoxy-(1-heptenyl)- silane (11) with 4-Iodoanisole}

Following General Procedure V, 8 (40.4 mg, 0.20 mmol), 11 (52.0 mg, 0.20 mmol), 4iodoanisole $(46.8 \mathrm{mg}, 0.20 \mathrm{mmol})$, a solution of naphthalene $(0.8 \mathrm{~mL}, 0.20 \mathrm{mmol}, 0.25 \mathrm{M}$ in THF) and TBAF (0.8 mL, $0.80 \mathrm{mmol}, 1.0 \mathrm{M}$ in THF) was stirred for $1 \mathrm{~h}$ and $\mathrm{Pd}(\mathrm{dba})_{2}(5.8 \mathrm{mg}$ $0.01 \mathrm{mmol}$ ) was added. The reaction was stirred at room temperature for $1 \mathrm{~h}$ and then two sample aliquots were taken and analyzed twice on GC. GC analysis of samples showed a 14/15 ratio of 76.4/23.6.

GC Data:

\begin{tabular}{l|c|c|c|c|c|}
\cline { 2 - 6 } reaction 1 & area naphth & area 14 & area 15 & response factor 14/15 & ratio 14/15x100 \\
\hline sample 1 & 49082 & 28098 & 10621 & $0.96 / 0.79$ & $76.3 / 23.7$ \\
\hline & 46729 & 27132 & 10294 & $0.96 / 0.79$ & $76.2 / 23.8$ \\
\hline sample 2 & 48724 & 28213 & 10717 & $0.96 / 0.79$ & $76.2 / 23.8$ \\
\hline & 47371 & 27367 & 10313 & $0.96 / 0.79$ & $76.4 / 23.6$ \\
\hline average & 47977 & 27703 & 10486 & $0.96 / 0.79$ & $76.3 / 23.7$ \\
\hline
\end{tabular}




\begin{tabular}{l|c|c|c|c|c|}
\cline { 2 - 6 } reaction 2 & area naphth & area 14 & area 15 & response factor 14/15 & ratio 14/15x100 \\
\hline sample 1 & 48650 & 30306 & 11392 & $0.96 / 0.79$ & $76.4 / 23.6$ \\
\hline & 49689 & 30847 & 11457 & $0.96 / 0.79$ & $76.6 / 23.4$ \\
\hline sample 2 & 49986 & 31218 & 11555 & $0.96 / 0.79$ & $76.7 / 23.3$ \\
\hline & 49589 & 30895 & 11566 & $0.96 / 0.79$ & $76.5 / 23.5$ \\
\hline average & 49479 & 30817 & 11493 & $0.96 / 0.79$ & $76.6 / 23.4$ \\
\hline
\end{tabular}

Competition of $(E)$-Dimethyl-(1-pentenyl)silanol (1) vs (E)- Dimethyl-(1-heptenyl)ethoxysilane (9) with 4-Iodoanisole

Following General Procedure V, 1 (28.8 mg, $0.20 \mathrm{mmol}), 9(40.0 \mathrm{mg}, 0.20 \mathrm{mmol}), 4-$ iodoanisole $(46.8 \mathrm{mg}, 0.20 \mathrm{mmol})$, a solution of naphthalene $(0.8 \mathrm{~mL}, 0.20 \mathrm{mmol}, 0.25 \mathrm{M}$ in THF) and TBAF (0.8 mL, $0.80 \mathrm{mmol}, 1.0 \mathrm{M}$ in THF) was stirred for $1 \mathrm{~h}$ and Pd(dba) $2(5.8 \mathrm{mg}$ $0.01 \mathrm{mmol}$ ) was added. The reaction was stirred at room temperature for $1 \mathrm{~h}$ and then two sample aliquots were taken and analyzed twice on GC. GC analysis of samples showed a 14/15 ratio of $49.4 / 50.6$.

GC Data:

\begin{tabular}{l|c|c|c|c|c|}
\cline { 2 - 6 } reaction 1 & area naphth & area 14 & area 15 & response factor 14/15 & ratio 14/15x100 \\
\hline sample 1 & 55280 & 29310 & 33723 & $0.96 / 0.79$ & $53.8 / 46.2$ \\
\hline & 51732 & 30201 & 40037 & $0.96 / 0.79$ & $48.7 / 51.3$ \\
\hline sample 2 & 136226 & 66353 & 78334 & $0.96 / 0.79$ & $50.8 / 49.2$ \\
\hline & 103450 & 61348 & 79557 & $0.96 / 0.79$ & $51.4 / 48.6$ \\
\hline average & 86672 & 46803 & 57913 & $0.96 / 0.79$ & $51.2 / 48.8$ \\
\hline
\end{tabular}

\begin{tabular}{l|c|c|c|c|c|}
\cline { 2 - 6 } reaction 2 & area naphth & area 14 & area 15 & response factor 14/15 & ratio 14/15x100 \\
\hline sample 1 & 36181 & 19548 & 25300 & $0.96 / 0.79$ & $46.8 / 53.2$ \\
\hline & 33750 & 16723 & 22760 & $0.96 / 0.79$ & $47.8 / 52.2$ \\
\hline sample 2 & 51360 & 22762 & 23731 & $0.96 / 0.79$ & $48.4 / 51.6$ \\
\hline & 33920 & 19852 & 27421 & $0.96 / 0.79$ & $47.2 / 52.8$ \\
\hline average & 38803 & 19721 & 24803 & $0.96 / 0.79$ & $47.6 / 52.4$ \\
\hline
\end{tabular}




\section{Competition Experiments with 2-Iodotoluene. General Procedure VI.}

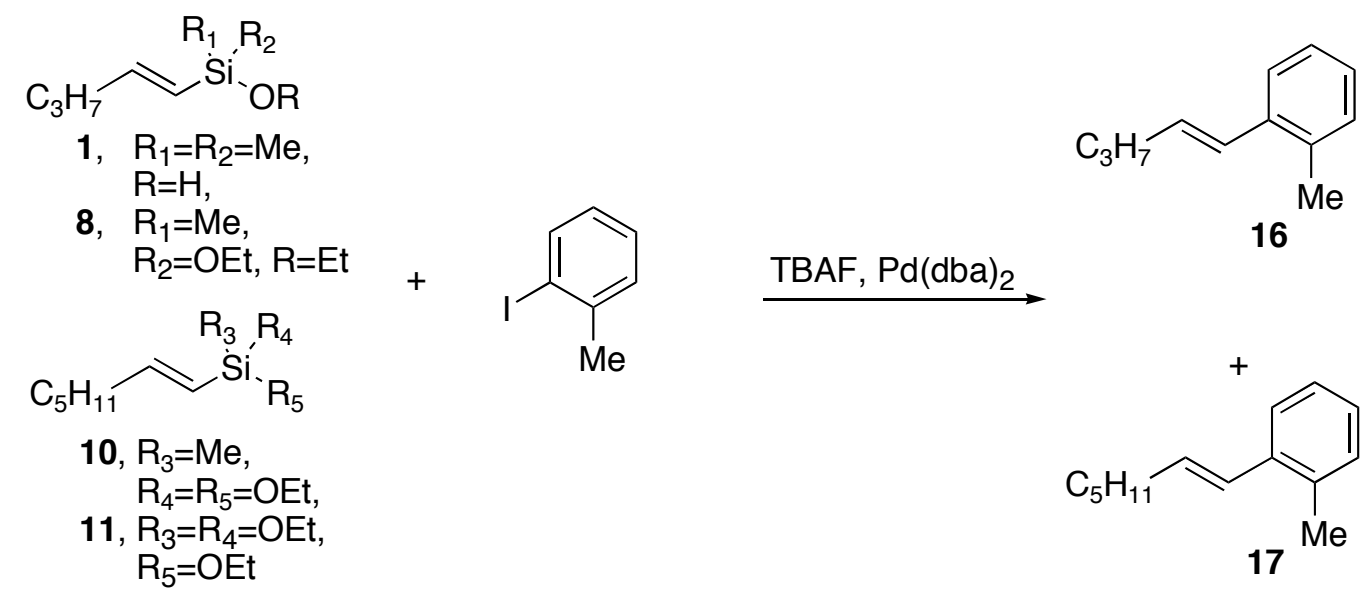

A flame-dried, 5-mL, 2-neck, round-bottomed flask under $\mathrm{N}_{2}$ was charged with (E)dimethyl-(1-pentenyl)silanol (1) or (E)-diethoxy-(1-pentenyl)methylsilane (8), $(E)$-triethoxy-(1heptenyl)silane (10) or (E)-diethoxy-(1-heptenyl)methylsilane (11), and 2-iodotoluene. THF solutions of naphthalene $(0.25 \mathrm{M})$ and TBAF $(1.0 \mathrm{M})$ were added next. The mixture was stirred at room temperature for $1 \mathrm{~h}$ and $\mathrm{Pd}(\mathrm{dba})_{2}$ was added. After $30 \mathrm{~min}$ two $25-\mu \mathrm{L}$ samples were taken via syringe. The sample aliquots were filtered through a plug of silica gel washing with $\mathrm{Et}_{2} \mathrm{O}$ to achieve a total sample volume of $\sim 2 \mathrm{~mL}$. These samples were then subjected to GC analysis. Reactions were performed in duplicate.

\section{Competition of $(E)$-Dimethyl-(1-pentenyl)silanol (1) vs (E)-Diethoxy-(1-heptenyl)methyl- silane (10) with 2-Iodotoluene}

Following General Procedure VI, 1 (28.8 mg, $0.20 \mathrm{mmol}), 10$ (46.0 mg, $0.20 \mathrm{mmol})$, 2iodotoluene $(25.6 \mu \mathrm{L}, 0.20 \mathrm{mmol})$, a solution of naphthalene $(0.8 \mathrm{~mL}, 0.20 \mathrm{mmol}, 0.25 \mathrm{M}$ in THF) and TBAF (0.8 mL, $0.80 \mathrm{mmol}, 1.0 \mathrm{M}$ in THF) was stirred for $1 \mathrm{~h}$ and $\mathrm{Pd}(\mathrm{dba}) 2(5.8 \mathrm{mg}$ $0.01 \mathrm{mmol}$ ) was added. The reaction was stirred at room temperature for $1 \mathrm{~h}$ and then two sample aliquots were taken and analyzed twice on GC. GC analysis of samples showed a 16/17 ratio of 51.4/48.6. 
GC Data:

\begin{tabular}{l|c|c|c|c|c|}
\cline { 2 - 6 } reaction 1 & area naphth & area 16 & area 17 & response factor 16/17 & ratio 16/17x100 \\
\hline sample 1 & 40099 & 23444 & 18578 & $0.86 / 0.71$ & $51.1 / 48.9$ \\
\hline & 17578 & 10609 & 9030 & $0.86 / 0.71$ & $49.3 / 50.7$ \\
\hline sample 2 & 34377 & 20022 & 15754 & $0.86 / 0.71$ & $51.3 / 48.7$ \\
\hline & 31432 & 18853 & 15953 & $0.86 / 0.71$ & $49.5 / 50.5$ \\
\hline average & 30872 & 18232 & 14829 & $0.86 / 0.71$ & $50.3 / 49.7$ \\
\hline
\end{tabular}

\begin{tabular}{l|c|c|c|c|c|}
\cline { 2 - 6 } reaction 2 & area naphth & area 16 & area 17 & response factor $16 / 17$ & ratio 16/17×100 \\
\hline sample 1 & 35409 & 19881 & 15110 & $0.86 / 0.71$ & $52.1 / 47.9$ \\
\hline & 36556 & 23764 & 18105 & $0.86 / 0.71$ & $52.1 / 47.9$ \\
\hline sample 2 & 50075 & 27983 & 20912 & $0.86 / 0.71$ & $52.6 / 47.4$ \\
\hline & 46599 & 26336 & 19575 & $0.86 / 0.71$ & $52.7 / 47.3$ \\
\hline average & 42160 & 24491 & 18426 & $0.86 / 0.71$ & $52.4 / 47.6$ \\
\hline
\end{tabular}

\section{Competition of $(E)$-Dimethyl-(1-pentenyl)silanol (1) vs (E)-Triethoxy-(1-heptenyl)silane} (11) with 2-Iodotoluene

Following General Procedure VI, 1 (28.8 mg, $0.20 \mathrm{mmol}), 14$ (52.0 mg, $0.20 \mathrm{mmol})$, 2iodotoluene $(25.6 \mu \mathrm{L}, 0.20 \mathrm{mmol})$, a solution of naphthalene $(0.8 \mathrm{~mL}, 0.20 \mathrm{mmol}, 0.25 \mathrm{M}$ in $\mathrm{THF})$ and TBAF $\left(0.8 \mathrm{~mL}, 0.80 \mathrm{mmol}, 1.0 \mathrm{M}\right.$ in THF) was stirred for $1 \mathrm{~h}$ and $\mathrm{Pd}(\mathrm{dba})_{2}(5.8 \mathrm{mg}$ $0.01 \mathrm{mmol}$ ) was added. The reaction was stirred at room temperature for $1 \mathrm{~h}$ and then two sample aliquots were taken and analyzed twice on GC. GC analysis of samples showed a 16/17 ratio of $81.1 / 18.9$

GC Data:

\begin{tabular}{l|c|c|c|c|c|}
\cline { 2 - 6 } reaction 1 & area naphth & area 16 & area 17 & response factor $16 / 17$ & ratio 16/17x100 \\
\hline sample 1 & 65559 & 41279 & 11396 & $0.86 / 0.71$ & $81.4 / 18.6$ \\
\hline & 64535 & 40389 & 11127 & $0.86 / 0.71$ & $81.4 / 18.6$ \\
\hline sample 2 & 49213 & 30732 & 8575 & $0.86 / 0.71$ & $81.2 / 18.8$ \\
\hline & 49089 & 30672 & 8514 & $0.86 / 0.71$ & $81.3 / 18.7$ \\
\hline average & 57099 & 35768 & 9903 & $0.86 / 0.71$ & $81.3 / 18.7$ \\
\hline
\end{tabular}




\begin{tabular}{l|c|c|c|c|c|}
\cline { 2 - 6 } reaction 2 & area naphth & area 16 & area 17 & response factor $16 / 17$ & ratio $16 / 17 \times 100$ \\
\hline sample 1 & 57551 & 41549 & 11813 & $0.86 / 0.71$ & $80.9 / 19.1$ \\
\hline & 58493 & 42322 & 12015 & $0.86 / 0.71$ & $80.9 / 19.1$ \\
\hline sample 2 & 50523 & 36321 & 10311 & $0.86 / 0.71$ & $80.9 / 19.1$ \\
\hline & 51554 & 37040 & 10517 & $0.86 / 0.71$ & $81.0 / 19.0$ \\
\hline average & 54530 & 39308 & 11164 & $0.86 / 0.71$ & $80.9 / 19.1$ \\
\hline
\end{tabular}

Competition of $(E)$ - Diethoxy-(1-pentenyl)methylsilane (8) vs (E)-Triethoxy-(1-heptenyl)silane (11) with 2-Iodotoluene

Following General Procedure VI, 8 (40.4 mg, $0.20 \mathrm{mmol}), 11(52.0 \mathrm{mg}, 0.20 \mathrm{mmol}), 2-$ iodotoluene $(25.6 \mu \mathrm{L}, 0.20 \mathrm{mmol})$, a solution of naphthalene $(0.8 \mathrm{~mL}, 0.20 \mathrm{mmol}, 0.25 \mathrm{M}$ in THF) and TBAF (0.8 mL, $0.80 \mathrm{mmol}, 1.0 \mathrm{M}$ in THF) was stirred for $1 \mathrm{~h}$ and $\mathrm{Pd}(\mathrm{dba})_{2}(5.8 \mathrm{mg}$ $0.01 \mathrm{mmol}$ ) was added. The reaction was stirred at room temperature for $1 \mathrm{~h}$ and then two sample aliquots were taken and analyzed twice on GC. GC analysis of samples showed a 16/17 ratio of 74.4/25.6.

GC Data:

\begin{tabular}{l|c|c|c|c|c|}
\cline { 2 - 6 } reaction 1 & area naphth & area 16 & area 17 & response factor 16/17 & ratio 16/17x100 \\
\hline sample 1 & 60499 & 46284 & 19176 & $0.86 / 0.71$ & $74.4 / 25.6$ \\
\hline & 63396 & 48347 & 19938 & $0.86 / 0.71$ & $74.2 / 25.8$ \\
\hline sample 2 & 49896 & 33954 & 14214 & $0.86 / 0.71$ & $74.2 / 25.8$ \\
\hline & 51629 & 34852 & 14214 & $0.86 / 0.71$ & $74.7 / 25.3$ \\
\hline average & 56355 & 40859 & 16886 & $0.86 / 0.71$ & $74.4 / 25.6$ \\
\hline
\end{tabular}

\begin{tabular}{l|c|c|c|c|c|}
\cline { 2 - 6 } reaction 2 & area naphth & area 16 & area 17 & response factor 16/17 & ratio 16/17x100 \\
\hline sample 1 & 55396 & 37786 & 15739 & $0.86 / 0.71$ & $74.3 / 25.7$ \\
\hline & 55300 & 37694 & 15739 & $0.86 / 0.71$ & $74.3 / 25.7$ \\
\hline sample 2 & 40863 & 31296 & 13097 & $0.86 / 0.71$ & $74.2 / 25.8$ \\
\hline & 40115 & 30675 & 12811 & $0.86 / 0.71$ & $74.3 / 25.7$ \\
\hline average & 47919 & 34363 & 14347 & $0.86 / 0.71$ & $74.3 / 25.7$ \\
\hline
\end{tabular}




\section{Competition Experiments with Potassium Trimethylsilanolate as Activator.}

Competition Experiments with Carbon Substituents in the Presence of Potassium Trimethylsilanolate. General Procedure VII.
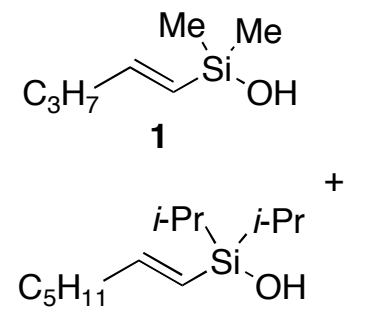

4

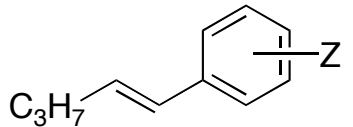

12, $\mathrm{Z}=4-\mathrm{MeCO}$

14, $Z=4-\mathrm{MeO}$

$16, Z=2-M e$

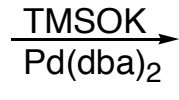

13, $\mathrm{Z}=4-\mathrm{MeCO}$

15, $Z=4-\mathrm{MeO}$

$17, Z=2-M e$

A flame-dried, 5-mL, 2-neck, round-bottomed flask under $\mathrm{N}_{2}$ was charged with $(E)$ dimethyl-(1-pentenyl)silanol (1), di(1-methylethyl)-(1-heptenyl)silanol (4) and the aryl iodide. A THF solution of naphthalene $(0.25 \mathrm{M})$ was added next followed by TMSOK and $\operatorname{Pd}(\mathrm{dba})_{2}$. After an appropriate time, two $25-\mu \mathrm{L}$ samples were taken via syringe. The sample aliquots were filtered through a plug of silica gel washing with $\mathrm{Et}_{2} \mathrm{O}$ to achieve a total sample volume of $\sim 2$ $\mathrm{mL}$. These samples were then subjected to GC analysis. Reactions were performed in duplicate.

Competition of $(E)$-Dimethyl-(1-pentenyl)silanol (1) vs (E)-Diisopropyl-(1-heptenyl)silanol (4) with 4-Iodoacetophenone

Following General Procedure VII, 1 (28.8 mg, $0.20 \mathrm{mmol}), 4$ (45.6 mg, $0.20 \mathrm{mmol})$, 4iodoacetophenone $(49.2 \mathrm{mg}, 0.20 \mathrm{mmol})$, a solution of naphthalene $(0.8 \mathrm{~mL}, 0.20 \mathrm{mmol}, 0.25 \mathrm{M}$ in DME) and TMSOK (25.8 mg, $0.80 \mathrm{mmol})$ and $\mathrm{Pd}(\mathrm{dba})_{2}(5.8 \mathrm{mg} 0.01 \mathrm{mmol})$ were stirred at room temperature for $14 \mathrm{~h}$ and then two sample aliquots were taken and analyzed twice on GC. GC analysis of samples showed a $\mathbf{1 2 / 1 3}$ ratio of 100/0. 
GC Data:

\begin{tabular}{l|c|c|c|c|c|}
\cline { 2 - 6 } reaction 1 & area naphth & area 12 & area 13 & response factor 12/13 & ratio 12/13x100 \\
\hline sample 1 & 8109 & 7911 & 0 & $0.78 / 0.67$ & $100 / 0$ \\
\hline & 7088 & 7579 & 0 & $0.78 / 0.67$ & $100 / 0$ \\
\hline sample 2 & 10186 & 9874 & 0 & $0.78 / 0.67$ & $100 / 0$ \\
\hline & 9290 & 9418 & 0 & $0.78 / 0.67$ & $100 / 0$ \\
\hline average & 8668 & 8696 & 0 & $0.78 / 0.67$ & $100.0 / 0.0$ \\
\hline
\end{tabular}

\begin{tabular}{|c|c|c|c|c|c|}
\hline reaction 2 & area naphth & area 12 & area 13 & response factor $12 / 13$ & ratio $12 / 13 \times 100$ \\
\hline \multirow[t]{2}{*}{ sample 1} & 9034 & 1325 & 0 & $0.78 / 0.67$ & $100 / 0$ \\
\hline & 7985 & 10105 & 0 & $0.78 / 0.67$ & $100 / 0$ \\
\hline \multirow[t]{2}{*}{ sample 2} & 9303 & 11746 & 0 & $0.78 / 0.67$ & $100 / 0$ \\
\hline & 9312 & 11335 & 0 & $0.78 / 0.67$ & $100 / 0$ \\
\hline average & 8909 & 8628 & 0 & $0.78 / 0.67$ & $100.0 / 0.0$ \\
\hline
\end{tabular}

\section{Competition of $(E)$-Dimethyl-(1-pentenyl)silanol (1) vs (E)-Di(1-methylsethy)-(1 heptenyl)- silanol (4) with 2-Iodotoluene}

Following General Procedure VII, 1 (28.8 mg, $0.20 \mathrm{mmol}), 4$ (45.6 mg, $0.20 \mathrm{mmol})$, 2iodotoluene $(25.6 \mu \mathrm{L}, 0.20 \mathrm{mmol})$, a solution of naphthalene $(0.8 \mathrm{~mL}, 0.20 \mathrm{mmol}, 0.25 \mathrm{M}$ in DME) and TMSOK $(25.8 \mathrm{mg}, 0.80 \mathrm{mmol})$ and $\mathrm{Pd}(\mathrm{dba})_{2}(5.8 \mathrm{mg} 0.01 \mathrm{mmol})$ were stirred at room temperature for $4 \mathrm{~h}$ and then two sample aliquots were taken and analyzed twice on GC. GC analysis of samples showed a $\mathbf{1 6 / 1 7}$ ratio of 93.7/6.3.

GC Data:

\begin{tabular}{l|c|c|c|c|c|}
\cline { 2 - 6 } reaction 1 & area naphth & area 16 & area 17 & response factor 16/17 & ratio 16/17×100 \\
\hline sample 1 & 16988 & 12745 & 947 & $0.78 / 0.67$ & $94.2 / 5.8$ \\
\hline & 15051 & 10791 & 801 & $0.78 / 0.67$ & $94.2 / 5.8$ \\
\hline sample 2 & 13632 & 10101 & 962 & $0.78 / 0.67$ & $92.7 / 7.3$ \\
\hline & 13838 & 10258 & 981 & $0.78 / 0.67$ & $92.7 / 7.3$ \\
\hline average & 14877 & 10974 & 923 & $0.78 / 0.67$ & $93.5 / 6.5$ \\
\hline
\end{tabular}




\begin{tabular}{l|c|c|c|c|c|}
\cline { 2 - 6 } reaction 2 & area naphth & area 16 & area 17 & response factor 16/17 & ratio 16/17×100 \\
\hline sample 1 & 14763 & 13054 & 1042 & $0.78 / 0.67$ & $93.8 / 6.8$ \\
\hline & 14012 & 12536 & 1002 & $0.78 / 0.67$ & $93.7 / 6.3$ \\
\hline sample 2 & 22285 & 19442 & 1322 & $0.78 / 0.67$ & $94.7 / 5.4$ \\
\hline & 19526 & 17396 & 1371 & $0.78 / 0.67$ & $93.9 / 6.1$ \\
\hline average & 17647 & 15607 & 1184 & $0.78 / 0.67$ & $93.9 / 6.1$ \\
\hline
\end{tabular}

\section{Competition of (E)-Dimethyl-(1-pentenyl)silanol (1) vs (E)-Di-(1-methylethyl)-(1 heptenyl)- silanol (4) with 4-Iodoanisole}

Following General Procedure VII, 1 (28.8 mg, $0.20 \mathrm{mmol}), 4(45.6 \mathrm{mg}, 0.20 \mathrm{mmol})$, 4iodoanisole $(46.8 \mathrm{mg}, 0.20 \mathrm{mmol})$, a solution of naphthalene $(0.8 \mathrm{~mL}, 0.20 \mathrm{mmol}, 0.25 \mathrm{M}$ in DME) and TMSOK (25.8 $\mathrm{mg}, 0.80 \mathrm{mmol})$ and $\mathrm{Pd}(\mathrm{dba})_{2}(5.8 \mathrm{mg} 0.01 \mathrm{mmol})$ were stirred at room temperature for $4 \mathrm{~h}$ and then two sample aliquots were taken and analyzed twice on GC. GC analysis of samples showed a 14/15 ratio of 95.0/5.0.

GC Data:

\begin{tabular}{l|c|c|c|c|c|}
\cline { 2 - 6 } reaction 1 & area naphth & area 14 & area 15 & response factor 14/15 & ratio 14/15x100 \\
\hline sample 1 & 10177 & 8386 & 663 & $0.96 / 0.79$ & $93.9 / 6.0$ \\
\hline & 11986 & 9371 & 642 & $0.96 / 0.79$ & $94.7 / 5.3$ \\
\hline sample 2 & 14510 & 10237 & 549 & $0.96 / 0.79$ & $95.8 / 4.2$ \\
\hline average & 13521 & 10010 & 545 & $0.96 / 0.79$ & $95.7 / 4.3$ \\
\hline
\end{tabular}

\begin{tabular}{|c|c|c|c|c|c|}
\hline reaction 2 & area naphth & area 14 & area 15 & response factor $14 / 15$ & ratio $14 / 15 \times 100$ \\
\hline \multirow[t]{2}{*}{ sample 1} & 12820 & 9712 & 609 & $0.96 / 0.79$ & $95.1 / 4.9$ \\
\hline & 14275 & 10540 & 650 & $0.96 / 0.79$ & $95.2 / 4.8$ \\
\hline \multirow[t]{2}{*}{ sample 2} & 15035 & 8525 & 498 & $0.96 / 0.79$ & $95.4 / 4.6$ \\
\hline & 12941 & 7787 & 543 & $0.96 / 0.79$ & $94.6 / 5.4$ \\
\hline average & 13768 & 9141 & 575 & $0.96 / 0.79$ & $95.1 / 4.9$ \\
\hline
\end{tabular}


Competition Experiments with 4-Iodoanisole in the Presence of Potassium Trimethylsilanolate. General Procedure VIII.

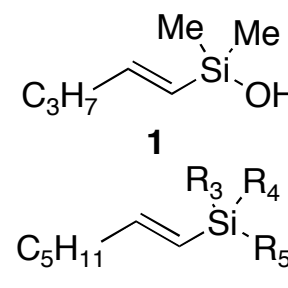

6, $R_{3}=R_{4}=P h$, $\mathrm{R}_{5}=\mathrm{OH}$,

7, $\mathrm{R}_{3}=\mathrm{CH}_{2} \mathrm{CF}_{3}$, $\mathrm{R}_{4}=\mathrm{Me}, \mathrm{R}_{5}=\mathrm{OH}$

9, $\mathrm{R}_{3}=\mathrm{R}_{4}=\mathrm{Me}$, $R_{5}=\mathrm{OEt}$,

10, $\mathrm{R}_{3}=\mathrm{Me}$, $\mathrm{R}_{4}=\mathrm{R}_{5}=\mathrm{OEt}$

11, $\mathrm{R}_{3}=\mathrm{R}_{4}=\mathrm{OEt}$, $\mathrm{R}_{5}=\mathrm{OEt}$

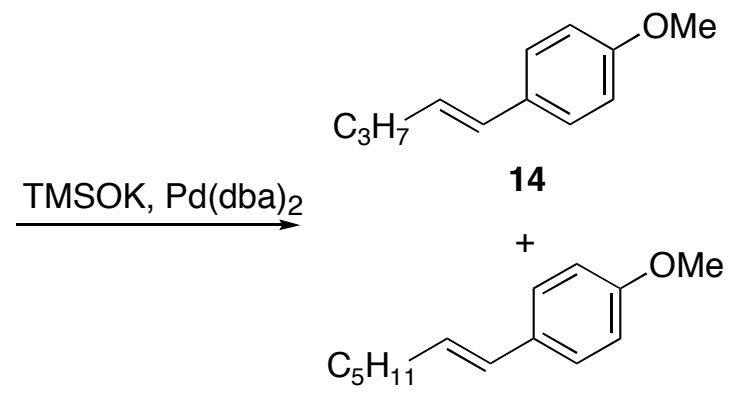

15

A flame-dried, 5-mL, 2-neck, round-bottomed flask under $\mathrm{N}_{2}$ was charged with $(E)$ dimethyl-(1-pentenyl)silanol (1), one of the (1-heptenyl)silanols (6-11) and 4-iodoanisole. A THF solution of naphthalene $(0.25 \mathrm{M})$ was added next followed by TMSOK and $\mathrm{Pd}(\mathrm{dba})_{2}$. After $4 \mathrm{~h}$, two $25-\mu \mathrm{L}$ samples were taken via syringe. The sample aliquots were filtered through a plug of silica gel washing with $\mathrm{Et}_{2} \mathrm{O}$ to achieve a total sample volume of $\sim 2 \mathrm{~mL}$. These samples were then subjected to GC analysis. Reactions were performed in duplicate.

\section{Competition of $(E)$-Dimethyl-(1-pentenyl)silanol (1) vs (E)-Diphenyl-(1 heptenyl)silanol (6)} with 4-Iodoanisole

Following General Procedure VIII, 1 (28.8 mg, $0.20 \mathrm{mmol}), 6$ (59.2 mg, $0.20 \mathrm{mmol})$, 4iodoanisole $(46.8 \mathrm{mg}, 0.20 \mathrm{mmol})$, a solution of naphthalene $(0.8 \mathrm{~mL}, 0.20 \mathrm{mmol}, 0.25 \mathrm{M}$ in THF) and TMSOK (102 mg, $0.80 \mathrm{mmol})$ and $\mathrm{Pd}(\mathrm{dba})_{2}(5.8 \mathrm{mg} 0.01 \mathrm{mmol})$ were stirred at room temperature for $4 \mathrm{~h}$ and then two sample aliquots were taken and analyzed twice on GC. GC analysis of samples showed a $\mathbf{1 4 / 1 5}$ ratio of 20.6/79.4. 
GC Data:

\begin{tabular}{l|c|c|c|c|c|}
\cline { 2 - 6 } reaction 1 & area naphth & area 14 & area 15 & response factor 14/15 & ratio 14/15x100 \\
\hline sample 1 & 10920 & 2036 & 10383 & $0.96 / 0.79$ & $19.3 / 80.7$ \\
\hline & 13224 & 2111 & 10117 & $0.96 / 0.79$ & $20.3 / 79.7$ \\
\hline sample 2 & 12746 & 2744 & 12088 & $0.96 / 0.79$ & $21.6 / 78.4$ \\
\hline average & 10889 & 2812 & 11887 & $0.96 / 0.79$ & $22.4 / 77.6$ \\
\hline
\end{tabular}

\begin{tabular}{l|c|c|c|c|c|}
\cline { 2 - 6 } reaction 2 & area naphth & area 14 & area 15 & response factor 14/15 & ratio 14/15×100 \\
\hline sample 1 & 27002 & 5281 & 25181 & $0.96 / 0.79$ & $20.3 / 79.7$ \\
\hline & 22444 & 4990 & 24059 & $0.96 / 0.79$ & $20.2 / 79.8$ \\
\hline sample 2 & 21416 & 4494 & 22861 & $0.96 / 0.79$ & $19.3 / 80.7$ \\
\hline & 23732 & 5307 & 23967 & $0.96 / 0.79$ & $21.2 / 78.8$ \\
\hline average & 23649 & 5018 & 24017 & $0.96 / 0.79$ & $20.3 / 79.7$ \\
\hline
\end{tabular}

\section{Competition of (E)-Dimethyl-(1-pentenyl)silanol (1) vs (E)-Trifluoropropyl-(1-heptenyl)- methylsilanol (7) with 4-Iodoanisole}

Following General Procedure VIII, 1 (28.8 mg, $0.20 \mathrm{mmol}), 7$ (50.8 mg, $0.20 \mathrm{mmol}), 4-$ iodoanisole $(46.8 \mathrm{mg}, 0.20 \mathrm{mmol})$, a solution of naphthalene $(0.8 \mathrm{~mL}, 0.20 \mathrm{mmol}, 0.25 \mathrm{M}$ in THF) and TMSOK (102 mg, $0.80 \mathrm{mmol})$ were stirred for $1 \mathrm{~h}$ and $\mathrm{Pd}(\mathrm{dba})_{2}(5.8 \mathrm{mg} 0.01 \mathrm{mmol})$ was added. The reaction was stirred at room temperature for $4 \mathrm{~h}$ and then two sample aliquots were taken and analyzed twice on GC. GC analysis of samples showed a 14/15 ratio of 34.9/65.1.

GC Data:

\begin{tabular}{l|c|c|c|c|c|}
\cline { 2 - 6 } reaction 1 & area naphth & area 14 & area 15 & response factor 14/15 & ratio 14/15×100 \\
\hline sample 1 & 9213 & 3254 & 7782 & $0.96 / 0.79$ & $33.7 / 66.3$ \\
\hline sample 2 & 10920 & 3618 & 7965 & $0.96 / 0.79$ & $35.6 / 64.4$ \\
\hline & 18740 & 5826 & 12499 & $0.96 / 0.79$ & $36.2 / 63.8$ \\
\hline average & 14974 & 5708 & 12352 & $0.96 / 0.79$ & $36.0 / 64.0$ \\
\hline
\end{tabular}




\begin{tabular}{l|c|c|c|c|c|}
\cline { 2 - 6 } reaction 2 & area naphth & area 14 & area 15 & response factor 14/15 & ratio 14/15×100 \\
\hline sample 1 & 15913 & 4443 & 10072 & $0.96 / 0.79$ & $34.9 / 65.1$ \\
\hline & 14890 & 4399 & 10118 & $0.96 / 0.79$ & $34.6 / 65.4$ \\
\hline sample 2 & 16420 & 4736 & 10709 & $0.96 / 0.79$ & $35.0 / 65.0$ \\
\hline & 14129 & 4372 & 10713 & $0.96 / 0.79$ & $33.1 / 66.9$ \\
\hline average & 15338 & 4488 & 10403 & $0.96 / 0.79$ & $34.4 / 65.6$ \\
\hline
\end{tabular}

\section{Competition of $(E)$-Dimethyl-(1-pentenyl)silanol (1) vs (E)- Dimethyl-(1-heptenyl) ethoxysilane (9) with 4-Iodoanisole}

Following General Procedure VIII, 1 (28.8 mg, $0.20 \mathrm{mmol}), 9$ (40.0 mg, $0.20 \mathrm{mmol})$, 4iodoanisole $(46.8 \mathrm{mg}, 0.20 \mathrm{mmol})$, a solution of naphthalene $(0.8 \mathrm{~mL}, 0.20 \mathrm{mmol}, 0.25 \mathrm{M}$ in THF) and TMSOK (102 mg, $0.80 \mathrm{mmol})$ were stirred for $1 \mathrm{~h}$ and $\mathrm{Pd}(\mathrm{dba})_{2}(5.8 \mathrm{mg} 0.01 \mathrm{mmol})$ was added. The reaction was stirred at room temperature for $4 \mathrm{~h}$ and then two sample aliquots were taken and analyzed twice on GC. GC analysis of samples showed a 14/15 ratio of 48.1/51.9.

GC Data:

\begin{tabular}{l|c|c|c|c|c|}
\cline { 2 - 6 } reaction 1 & area naphth & area 14 & area 15 & response factor 14/15 & ratio 14/15×100 \\
\hline sample 1 & 7822 & 3398 & 4320 & $0.96 / 0.79$ & $48.9 / 51.1$ \\
\hline & 5984 & 2763 & 4025 & $0.96 / 0.79$ & $45.5 / 54.4$ \\
\hline sample 2 & 5884 & 2883 & 4082 & $0.96 / 0.79$ & $46.2 / 53.7$ \\
\hline & 6834 & 2846 & 3733 & $0.96 / 0.79$ & $48.1 / 51.9$ \\
\hline average & 6631 & 2973 & 4040 & $0.96 / 0.79$ & $47.2 / 52.8$ \\
\hline
\end{tabular}

\begin{tabular}{l|c|c|c|c|c|}
\cline { 2 - 6 } reaction 2 & area naphth & area 14 & area 15 & response factor 14/15 & ratio 14/15×100 \\
\hline sample 1 & 14268 & 5150 & 6635 & $0.96 / 0.79$ & $48.6 / 51.4$ \\
\hline & 12609 & 4823 & 6132 & $0.96 / 0.79$ & $48.9 / 51.1$ \\
\hline sample 2 & 11388 & 3972 & 4765 & $0.96 / 0.79$ & $50.4 / 49.6$ \\
\hline & 8838 & 4555 & 5975 & $0.96 / 0.79$ & $48.1 / 51.9$ \\
\hline average & 11776 & 4625 & 5877 & $0.96 / 0.79$ & $49.0 / 51.0$ \\
\hline
\end{tabular}


Competition of $(E)$-Dimethyl-(1-pentenyl)silanol (1) vs (E)-Diethoxy-(1-heptenyl)methylsilane (10) with 4-Iodoanisole

Following General Procedure VIII, 1 (28.8 mg, 0.20 mmol), 10 (46.0 mg, 0.20 mmol), 4iodotoluene $(46.8 \mathrm{mg}, 0.20 \mathrm{mmol})$, a solution of naphthalene $(0.8 \mathrm{~mL}, 0.20 \mathrm{mmol}, 0.25 \mathrm{M}$ in THF) and TMSOK (102 mg, $0.80 \mathrm{mmol}, 4$ equiv) was stirred for $1 \mathrm{~h}$ and $\mathrm{Pd}(\mathrm{dba})_{2}(5.8 \mathrm{mg} 0.01$ mmol) was added. The reaction was stirred at room temperature for $4 \mathrm{~h}$ and then two sample aliquots were taken and analyzed twice on GC. GC analysis of samples showed a 14/15 ratio of 45.2/54.8.

GC Data:

\begin{tabular}{l|c|c|c|c|c|}
\cline { 2 - 6 } reaction 1 & area naphth & area 14 & area 15 & response factor 14/15 & ratio 14/15x100 \\
\hline sample 1 & 12383 & 5015 & 6820 & $0.96 / 0.79$ & $47.2 / 52.8$ \\
\hline & 14152 & 5473 & 7759 & $0.96 / 0.79$ & $46.2 / 53.8$ \\
\hline sample 2 & 14350 & 5591 & 7614 & $0.96 / 0.79$ & $47.2 / 52.8$ \\
\hline & 12685 & 5158 & 7438 & $0.96 / 0.79$ & $45.8 / 54.2$ \\
\hline average & 13393 & 5309 & 7408 & $0.96 / 0.79$ & $46.6 / 53.4$ \\
\hline
\end{tabular}

\begin{tabular}{l|c|c|c|c|c|}
\cline { 2 - 6 } reaction 2 & area naphth & area 14 & area 15 & response factor 14/15 & ratio 14/15x100 \\
\hline sample 1 & 10483 & 3694 & 5885 & $0.96 / 0.79$ & $43.3 / 56.7$ \\
\hline & 10944 & 3532 & 5508 & $0.96 / 0.79$ & $43.8 / 56.1$ \\
\hline sample 2 & 13787 & 4374 & 6477 & $0.96 / 0.79$ & $45.1 / 54.9$ \\
\hline & 11565 & 3684 & 5892 & $0.96 / 0.79$ & $43.2 / 56.8$ \\
\hline average & 11695 & 3821 & 5941 & $0.96 / 0.79$ & $43.9 / 56.1$ \\
\hline
\end{tabular}

\section{Competition of (E)-Dimethyl-(1-pentenyl)silanol (1) vs (E)-Triethoxy-(1-heptenyl)silane} (11) with 4-Iodoanisole

Following General Procedure VIII, 1 (28.8 mg, 0.20 mmol), 11 (52.0 mg, 0.20 mmol), 4iodoanisole $(46.8 \mathrm{mg}, 0.20 \mathrm{mmol})$, a solution of naphthalene $(0.8 \mathrm{~mL}, 0.20 \mathrm{mmol}, 0.25 \mathrm{M}$ in THF) and TMSOK (102 mg, $0.80 \mathrm{mmol}, 4$ equiv) were stirred for $1 \mathrm{~h}$ and $\mathrm{Pd}(\mathrm{dba})_{2}$ (5.8 $\mathrm{mg} 0.01$ mmol) was added. The reaction was stirred at room temperature for $4 \mathrm{~h}$ and then two sample aliquots were taken and analyzed twice on GC. GC analysis of samples showed a 14/15 ratio of 51.6/48.4. 
GC Data:

\begin{tabular}{l|c|c|c|c|c|}
\cline { 2 - 6 } reaction 1 & area naphth & area 14 & area 15 & response factor 14/15 & ratio 14/15x100 \\
\hline sample 1 & 20487 & 8602 & 10147 & $0.96 / 0.79$ & $50.8 / 49.2$ \\
\hline & 20825 & 11020 & 12209 & $0.96 / 0.79$ & $52.4 / 47.6$ \\
\hline sample 2 & 22197 & 9139 & 10343 & $0.96 / 0.79$ & $51.8 / 48.2$ \\
\hline & 20003 & 8185 & 9599 & $0.96 / 0.79$ & $50.9 / 49.1$ \\
\hline average & 20878 & 9237 & 10575 & $0.96 / 0.79$ & $51.5 / 48.5$ \\
\hline
\end{tabular}

\begin{tabular}{l|c|c|c|c|c|}
\cline { 2 - 6 } reaction 2 & area naphth & area 14 & area 15 & response factor 14/15 & ratio 14/15x100 \\
\hline sample 1 & 19421 & 6903 & 7745 & $0.96 / 0.79$ & $52.0 / 48.0$ \\
\hline & 14983 & 6151 & 7832 & $0.96 / 0.79$ & $48.9 / 51.1$ \\
\hline sample 2 & 17814 & 7132 & 8018 & $0.96 / 0.79$ & $52.0 / 48.0$ \\
\hline & 17462 & 6857 & 7118 & $0.96 / 0.79$ & $53.8 / 46.2$ \\
\hline average & 17420 & 6761 & 7678 & $0.96 / 0.79$ & $51.7 / 48.3$ \\
\hline
\end{tabular}

\section{Competition Experiments of (E)-Dimethyl-(1-pentenyl)silanol (1) vs (E)-Di(1-methylethyl)-}

\section{(1-heptenyl)silanol (4) with 4-Iodoanisole. General Procedure IX.}<smiles>C[Si](C)(O)/C=C/c1ccccc1</smiles>

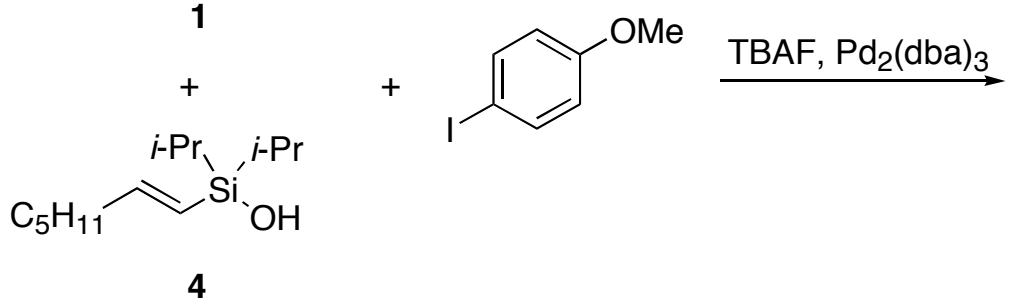<smiles>COc1ccc(/C=C/[13CH][13CH]c2ccccc2)cc1</smiles>

(E)-Dimethyl-(1-pentenyl)silanol (1), together with (E)-Di(1-methylethyl)-(1-heptenyl)silanol (4), naphthalene, and 4-iodoanisole were dissolved in a TBAF solution (1.0 M in THF) in a flame-dried, 5-mL, 2-neck, round-bottomed flask under $\mathrm{Ar} . \mathrm{Pd}_{2}(\mathrm{dba})_{3}$ was added next and the resulting mixture stirred for $1 \mathrm{~h}$. Two $25 \mu \mathrm{L}$ samples were then taken via syringe. The sample aliquots were filtered through a plug of silica gel washing with EtOAc to achieve a total sample volume of $\sim 2 \mathrm{~mL}$. These samples were then subjected to GC analysis. Reactions were performed in duplicate. 


\section{Competition of 1 equiv (E)-Dimethyl-(1-pentenyl)silanol (1) vs 1 equiv (E)-Di(1-} methylethyl)-(1-heptenyl)silanol (4) with 4-Iodoanisole and 1 equiv TBAF.

Following General Procedure IX, 1 (28.8 mg, 0.20 mmol), 4 (45.6 mg, 0.20 mmol), 4iodoanisole (46.8 mg, $0.20 \mathrm{mmol})$, naphthalene $(22.3 \mathrm{mg})$, TBAF $(0.2 \mathrm{~mL}, 0.20 \mathrm{mmol}, 1.0 \mathrm{M}$ in THF) and $\mathrm{Pd}_{2}(\mathrm{dba})_{3}(4.6 \mathrm{mg} 0.005 \mathrm{mmol})$ were stirred at room temperature for $1 \mathrm{~h}$ and then two sample aliquots were taken and analyzed twice on GC. GC analysis of samples showed a 14/15 ratio of 53.4/46.6.

GC Data:

\begin{tabular}{l|c|c|c|c|c|}
\cline { 2 - 6 } & area naphth & area 14 & area 15 & response factor 14/15 & ratio 14/15×100 \\
\hline sample 1 & 33357 & 14468 & 14037 & $0.87 / 0.79$ & $53.1 / 46.9$ \\
\hline sample 2 & 40130 & 16930 & 16170 & $0.87 / 0.79$ & $53.6 / 46.4$ \\
\hline average & 36744 & 15699 & 15104 & $0.87 / 0.79$ & $53.4 / 46.6$ \\
\hline
\end{tabular}

\section{Competition of 1 equiv (E)-Dimethyl-(1-pentenyl)silanol (1) vs 1 equiv (E)-Di(1- methylethyl)-(1-heptenyl)silanol (4) with 4-Iodoanisole and 1 equiv TBAF.}

Following General Procedure IX, 1 (28.8 mg, $0.20 \mathrm{mmol}), 4$ (45.6 mg, $0.20 \mathrm{mmol})$, 4iodoanisole (46.8 mg, $0.20 \mathrm{mmol})$, naphthalene (11.6 mg), TBAF $(0.2 \mathrm{~mL}, 0.20 \mathrm{mmol}, 1.0 \mathrm{M}$ in THF) and $\mathrm{Pd}_{2}(\mathrm{dba})_{3}(4.6 \mathrm{mg} 0.005 \mathrm{mmol})$ were stirred at room temperature for $1 \mathrm{~h}$ and then two sample aliquots were taken and analyzed twice on GC. GC analysis of samples showed a 14/15 ratio of 55.7/44.3.

GC Data:

\begin{tabular}{l|c|c|c|c|c|}
\cline { 2 - 6 } & area naphth & area 14 & area 15 & response factor 14/15 & ratio 14/15×100 \\
\hline sample 1 & 18797 & 12336 & 10723 & $0.87 / 0.79$ & $55.9 / 44.1$ \\
\hline sample 2 & 30774 & 19873 & 17691 & $0.87 / 0.79$ & $55.4 / 44.6$ \\
\hline average & 24745 & 16105 & 14207 & $0.87 / 0.79$ & $55.7 / 44.3$ \\
\hline
\end{tabular}

\section{Competition of 1 equiv (E)-Dimethyl-(1-pentenyl)silanol (1) vs 1 equiv (E)-Di(1-} methylethyl)-(1-heptenyl)silanol (4) with 4-Iodoanisole and 2 equiv TBAF.

Following General Procedure IX, 1 (28.8 mg, $0.20 \mathrm{mmol}), 4$ (45.6 mg, $0.20 \mathrm{mmol})$, 4iodoanisole (46.8 mg, $0.20 \mathrm{mmol})$, naphthalene (11.4 mg), TBAF (0.4 mL, $0.40 \mathrm{mmol}, 1.0 \mathrm{M}$ in THF) and $\mathrm{Pd}_{2}(\mathrm{dba})_{3}(4.6 \mathrm{mg} 0.005 \mathrm{mmol})$ were stirred at room temperature for $1 \mathrm{~h}$ and then two 
sample aliquots were taken and analyzed twice on GC. GC analysis of samples showed a 14/15 ratio of 58.5/41.5.

GC Data:

\begin{tabular}{l|c|c|c|c|c|}
\cline { 2 - 5 } & area naphth & area 14 & area 15 & response factor 14/15 & ratio 14/15x100 \\
\hline sample 1 & 25030 & 25148 & 19628 & $0.87 / 0.79$ & $58.5 / 41.5$ \\
\hline sample 2 & 26012 & 26279 & 20708 & $0.87 / 0.79$ & $58.4 / 41.6$ \\
\hline average & 25521 & 25714 & 20168 & $0.87 / 0.79$ & $58.5 / 41.5$ \\
\hline
\end{tabular}

\section{Competition of 1 equiv (E)-Dimethyl-(1-pentenyl)silanol (1) vs 1 equiv (E)-Di(1-} methylethyl)-(1-heptenyl)silanol (4) with 4-Iodoanisole and 2 equiv TBAF.

Following General Procedure IX, 1 (28.8 mg, $0.20 \mathrm{mmol}), 4$ (45.6 mg, $0.20 \mathrm{mmol})$, 4iodoanisole $(46.8 \mathrm{mg}, 0.20 \mathrm{mmol})$, naphthalene $(22.2 \mathrm{mg})$, TBAF $(0.4 \mathrm{~mL}, 0.40 \mathrm{mmol}, 1.0 \mathrm{M}$ in THF) and $\mathrm{Pd}_{2}(\mathrm{dba})_{3}(4.6 \mathrm{mg} 0.005 \mathrm{mmol})$ were stirred at room temperature for $1 \mathrm{~h}$ and then two sample aliquots were taken and analyzed twice on GC. GC analysis of samples showed a 14/15 ratio of 58.5/41.5.

GC Data:

\begin{tabular}{l|c|c|c|c|c|}
\cline { 2 - 6 } & area naphth & area 14 & area 15 & response factor 14/15 & ratio 14/15×100 \\
\hline sample 1 & 29357 & 18201 & 14864 & $0.87 / 0.79$ & $57.5 / 42.6$ \\
\hline sample 2 & 26383 & 14283 & 10732 & $0.87 / 0.79$ & $59.4 / 40.6$ \\
\hline average & 27870 & 16242 & 12798 & $0.87 / 0.79$ & $58.5 / 41.6$ \\
\hline
\end{tabular}

\section{Competition of 1 equiv ( $E$ )-Dimethyl-(1-pentenyl)silanol (1) vs 1 equiv (E)-Di(1-} methylethyl)-(1-heptenyl)silanol (4) with 4-Iodoanisole and 4 equiv TBAF.

Following General Procedure IX, 1 (28.8 mg, $0.20 \mathrm{mmol}), 4$ (45.6 mg, $0.20 \mathrm{mmol})$, 4iodoanisole $(46.8 \mathrm{mg}, 0.20 \mathrm{mmol})$, naphthalene $(20.8 \mathrm{mg})$, TBAF $(0.8 \mathrm{~mL}, 0.80 \mathrm{mmol}, 1.0 \mathrm{M}$ in $\mathrm{THF})$ and $\mathrm{Pd}_{2}(\mathrm{dba})_{3}(4.6 \mathrm{mg} 0.005 \mathrm{mmol})$ were stirred at room temperature for $1 \mathrm{~h}$ and then two sample aliquots were taken and analyzed twice on GC. GC analysis of samples showed a 14/15 ratio of $66.9 / 33.1$. 
GC Data:

\begin{tabular}{l|c|c|c|c|c|}
\cline { 2 - 5 } & area naphth & area 14 & area 15 & response factor 14/15 & ratio 14/15×100 \\
\hline sample 1 & 8147 & 4295 & 2282 & $0.87 / 0.79$ & $67.5 / 32.5$ \\
\hline sample 2 & 14041 & 7763 & 4282 & $0.87 / 0.79$ & $66.8 / 33.4$ \\
\hline average & 11094 & 6029 & 3282 & $0.87 / 0.79$ & $66.9 / 33.1$ \\
\hline
\end{tabular}

\section{Competition of 1 equiv (E)-Dimethyl-(1-pentenyl)silanol (1) vs 1 equiv (E)-Di(1-}

methylethyl)-(1-heptenyl)silanol (4) with 4-Iodoanisole and 4 equiv TBAF.

Following General Procedure IX, 1 (28.8 mg, $0.20 \mathrm{mmol}), 4$ (45.6 mg, $0.20 \mathrm{mmol})$, 4iodoanisole (46.8 mg, $0.20 \mathrm{mmol})$, naphthalene $(14.1 \mathrm{mg})$, TBAF $(0.8 \mathrm{~mL}, 0.80 \mathrm{mmol}, 1.0 \mathrm{M}$ in THF) and $\mathrm{Pd}_{2}(\mathrm{dba})_{3}(4.6 \mathrm{mg} 0.005 \mathrm{mmol})$ were stirred at room temperature for $1 \mathrm{~h}$ and then two sample aliquots were taken and analyzed twice on GC. GC analysis of samples showed a 14/15 ratio of $65.0 / 34.0$.

GC Data:

\begin{tabular}{l|c|c|c|c|c|}
\cline { 2 - 6 } & area naphth & area 14 & area 15 & response factor 14/15 & ratio 14/15×100 \\
\hline sample 1 & 10116 & 8167 & 4814 & $0.87 / 0.79$ & $65.2 / 34.8$ \\
\hline sample 2 & 10205 & 9091 & 5443 & $0.87 / 0.79$ & $64.9 / 35.1$ \\
\hline average & 10161 & 8629 & 5129 & $0.87 / 0.79$ & $65.1 / 34.9$ \\
\hline
\end{tabular}

\section{Competition of 1 equiv (E)-Dimethyl-(1-pentenyl)silanol (1) vs 1 equiv (E)-Di(1- methylethyl)-(1-heptenyl)silanol (4) with 4-Iodoanisole and 8 equiv TBAF.}

Following General Procedure IX, 1 (28.8 mg, $0.20 \mathrm{mmol}), 4$ (45.6 mg, $0.20 \mathrm{mmol})$, 4iodoanisole $(46.8 \mathrm{mg}, 0.20 \mathrm{mmol})$, naphthalene $(23.5 \mathrm{mg})$, TBAF (1.6 mL, 1.60 mmol, 1.0 M in $\mathrm{THF})$ and $\mathrm{Pd}_{2}(\mathrm{dba})_{3}(4.6 \mathrm{mg} 0.005 \mathrm{mmol})$ were stirred at room temperature for $1 \mathrm{~h}$ and then two sample aliquots were taken and analyzed twice on GC. GC analysis of samples showed a 14/15 ratio of $67.1 / 32.9$.

GC Data:

\begin{tabular}{l|c|c|c|c|c|}
\cline { 2 - 6 } & area naphth & area 14 & area 15 & response factor 14/15 & ratio 14/15x100 \\
\hline sample 1 & 21870 & 12164 & 6563 & $0.87 / 0.79$ & $67.1 / 32.9$ \\
\hline sample 2 & 6474 & 3705 & 2023 & $0.87 / 0.79$ & $67.1 / 32.9$ \\
\hline average & 14172 & 7935 & 4293 & $0.87 / 0.79$ & $67.1 / 32.9$ \\
\hline
\end{tabular}




\section{Competition of 1 equiv (E)-Dimethyl-(1-pentenyl)silanol (1) vs 1 equiv (E)-Di(1-} methylethyl)-(1-heptenyl)silanol (4) with 4-Iodoanisole and 8 equiv TBAF.

Following General Procedure IX, 1 (28.8 mg, 0.20 mmol), 4 (45.6 mg, 0.20 mmol), 4iodoanisole (46.8 mg, $0.20 \mathrm{mmol})$, naphthalene (14.5 mg), TBAF (1.6 mL, $1.60 \mathrm{mmol}, 1.0 \mathrm{M}$ in THF) and $\mathrm{Pd}_{2}(\mathrm{dba})_{3}(4.6 \mathrm{mg} 0.005 \mathrm{mmol})$ were stirred at room temperature for $1 \mathrm{~h}$ and then two sample aliquots were taken and analyzed twice on GC. GC analysis of samples showed a 14/15 ratio of 66.1/33.9.

GC Data:

\begin{tabular}{l|c|c|c|c|c|}
\cline { 2 - 6 } & area naphth & area 14 & area 15 & response factor 14/15 & ratio 14/15×100 \\
\hline sample 1 & 7962 & 4936 & 2450 & $0.87 / 0.79$ & $68.9 / 31.1$ \\
\hline sample 2 & 14725 & 10608 & 6836 & $0.87 / 0.79$ & $63.1 / 36.9$ \\
\hline average & 11344 & 7772 & 4643 & $0.87 / 0.79$ & $66.1 / 33.9$ \\
\hline
\end{tabular}

\section{Competition of 2 equiv (E)-Dimethyl-(1-pentenyl)silanol (1) vs 2 equiv (E)-Di(1-} methylethyl)-(1-heptenyl)silanol (4) with 4-Iodoanisole and 8 equiv TBAF.

Following General Procedure IX, 1 (28.8 mg, $0.20 \mathrm{mmol}), 4$ (45.6 mg, $0.20 \mathrm{mmol})$, 4iodoanisole $(23.4 \mathrm{mg}, 0.10 \mathrm{mmol})$, naphthalene $(10.3 \mathrm{mg})$, TBAF $(0.8 \mathrm{~mL}, 0.8 \mathrm{mmol}, 1.0 \mathrm{M}$ in THF) and $\mathrm{Pd}_{2}(\mathrm{dba})_{3}(2.3 \mathrm{mg} 0.0025 \mathrm{mmol})$ were stirred at room temperature for $1 \mathrm{~h}$ and then two sample aliquots were taken and analyzed twice on GC. GC analysis of samples showed a $\mathbf{1 4} / \mathbf{1 5}$ ratio of $68.0 / 32.0$.

GC Data:

\begin{tabular}{l|c|c|c|c|c|}
\cline { 2 - 6 } & area naphth & area 14 & area 15 & response factor 14/15 & ratio 14/15×100 \\
\hline sample 1 & 15634 & 8491 & 4485 & $0.87 / 0.79$ & $68.1 / 31.9$ \\
\hline sample 2 & 16164 & 9183 & 4751 & $0.87 / 0.79$ & $67.9 / 32.1$ \\
\hline average & 15899 & 8837 & 4618 & $0.87 / 0.79$ & $68.0 / 32.0$ \\
\hline
\end{tabular}

\section{Competition of 2 equiv (E)-Dimethyl-(1-pentenyl)silanol (1) vs 2 equiv (E)-Di(1-} methylethyl)-(1-heptenyl)silanol (4) with 4-Iodoanisole and 8 equiv TBAF.

Following General Procedure IX, 1 (28.8 mg, $0.20 \mathrm{mmol}), 4$ (45.6 mg, $0.20 \mathrm{mmol})$, 4iodoanisole (23.4 mg, $0.10 \mathrm{mmol})$, naphthalene $(11.0 \mathrm{mg})$, TBAF $(0.8 \mathrm{~mL}, 0.8 \mathrm{mmol}, 1.0 \mathrm{M}$ in THF) and $\mathrm{Pd}_{2}(\mathrm{dba})_{3}(2.3 \mathrm{mg} 0.0025 \mathrm{mmol})$ were stirred at room temperature for $1 \mathrm{~h}$ and then 
two sample aliquots were taken and analyzed twice on GC. GC analysis of samples showed a $\mathbf{1 4 / 1 5}$ ratio of $66.0 / 34.0$.

GC Data:

\begin{tabular}{l|c|c|c|c|c|}
\cline { 2 - 6 } & area naphth & area 14 & area 15 & response factor 14/15 & ratio 14/15×100 \\
\hline sample 1 & 12430 & 6762 & 3973 & $0.87 / 0.79$ & $65.3 / 34.7$ \\
\hline sample 2 & 11752 & 5981 & 3294 & $0.87 / 0.79$ & $66.7 / 33.4$ \\
\hline average & 12091 & 6372 & 3634 & $0.87 / 0.79$ & $66.0 / 34.0$ \\
\hline
\end{tabular}

\section{Competition of 3 equiv (E)-Dimethyl-(1-pentenyl)silanol (1) vs 3 equiv (E)-Di(1- methylethyl)-(1-heptenyl)silanol (4) with 4-Iodoanisole and 12 equiv TBAF.}

Following General Procedure IX, 1 (43.2 mg, $0.30 \mathrm{mmol}), 4$ (68.5 mg, $0.30 \mathrm{mmol})$, 4iodoanisole (23.4 mg, $0.10 \mathrm{mmol})$, naphthalene $(8.7 \mathrm{mg})$, TBAF (1.2 mL, $1.2 \mathrm{mmol}, 1.0 \mathrm{M}$ in THF) and $\mathrm{Pd}_{2}(\mathrm{dba})_{3}(2.3 \mathrm{mg} 0.0025 \mathrm{mmol})$ were stirred at room temperature for $1 \mathrm{~h}$ and then two sample aliquots were taken and analyzed twice on GC. GC analysis of samples showed a $\mathbf{1 4 / 1 5}$ ratio of $68.8 / 31.2$.

GC Data:

\begin{tabular}{l|c|c|c|c|c|}
\cline { 2 - 5 } & area naphth & area 14 & area 15 & response factor 14/15 & ratio 14/15×100 \\
\hline sample 1 & 7068 & 4567 & 2183 & $0.87 / 0.79$ & $69.8 / 30.2$ \\
\hline sample 2 & 12681 & 8735 & 4560 & $0.87 / 0.79$ & $67.9 / 32.1$ \\
\hline average & 9875 & 6651 & 3372 & $0.87 / 0.79$ & $68.8 / 31.2$ \\
\hline
\end{tabular}

\section{Competition of 3 equiv (E)-Dimethyl-(1-pentenyl)silanol (1) vs 3 equiv (E)-Di(1-} methylethyl)-(1-heptenyl)silanol (4) with 4-Iodoanisole and 12 equiv TBAF.

Following General Procedure IX, 1 (43.2 mg, $0.30 \mathrm{mmol}), 4$ (68.5 mg, $0.30 \mathrm{mmol})$, 4iodoanisole (23.4 mg, $0.10 \mathrm{mmol})$, naphthalene (10.3 mg), TBAF (1.2 mL, 1.2 mmol, 1.0 M in THF) and $\mathrm{Pd}_{2}(\mathrm{dba})_{3}(2.3 \mathrm{mg} 0.0025 \mathrm{mmol})$ were stirred at room temperature for $1 \mathrm{~h}$ and then two sample aliquots were taken and analyzed twice on GC. GC analysis of samples showed a $\mathbf{1 4} / \mathbf{1 5}$ ratio of $69.9 / 30.1$. 
GC Data:

\begin{tabular}{l|c|c|c|c|c|}
\cline { 2 - 6 } & area naphth & area 14 & area 15 & response factor 14/15 & ratio 14/15×100 \\
\hline sample 1 & 8751 & 5424 & 2666 & $0.87 / 0.79$ & $69.2 / 30.8$ \\
\hline sample 2 & 11933 & 7329 & 3381 & $0.87 / 0.79$ & $70.5 / 29.5$ \\
\hline average & 12091 & 6372 & 3634 & $0.87 / 0.79$ & $69.9 / 30.1$ \\
\hline
\end{tabular}

Competition of 1 equiv (E)-Dimethyl-(1-pentenyl)silanol (1) vs 1 equiv (E)-Di(1methylethyl)-(1-heptenyl)silanol (4) with 4-Iodoanisole and 1 equiv TMSOK.

Following General Procedure IX, 1 (28.8 mg, $0.20 \mathrm{mmol}), 4$ (45.6 mg, $0.20 \mathrm{mmol})$, 4iodoanisole (46.8 mg, $0.20 \mathrm{mmol})$, naphthalene $(20.5 \mathrm{mg})$, TMSOK (0.2 mL, $0.20 \mathrm{mmol}, 1.0 \mathrm{M}$ in THF ) and $\mathrm{Pd}_{2}(\mathrm{dba})_{3}(4.6 \mathrm{mg} 0.005 \mathrm{mmol})$ were stirred at room temperature for $1 \mathrm{~h}$ and then two sample aliquots were taken and analyzed twice on GC. GC analysis of samples showed a $\mathbf{1 4} / \mathbf{1 5}$ ratio of $1.0 / 0$.

GC Data:

\begin{tabular}{l|c|c|c|c|c|}
\cline { 2 - 6 } & area naphth & area 14 & area 15 & response factor 14/15 & ratio 14/15×100 \\
\hline sample 1 & 51446 & 13127 & 0 & $0.87 / 0.79$ & $1.0 / 0$ \\
\hline sample 2 & 44112 & 10900 & 0 & $0.87 / 0.79$ & $1.0 / 0$ \\
\hline average & 47779 & 12014 & 0 & $0.87 / 0.79$ & $1.0 / 0$ \\
\hline
\end{tabular}

\section{Competition of 1 equiv (E)-Dimethyl-(1-pentenyl)silanol (1) vs 1 equiv (E)-Di(1- methylethyl)-(1-heptenyl)silanol (4) with 4-Iodoanisole and 2 equiv TMSOK.}

Following General Procedure IX, 1 (28.8 mg, $0.20 \mathrm{mmol}), 4$ (45.6 mg, $0.20 \mathrm{mmol})$, 4iodoanisole (46.8 mg, $0.20 \mathrm{mmol})$, naphthalene (19.5 mg), TMSOK (0.4 mL, $0.40 \mathrm{mmol}, 1.0 \mathrm{M}$ in $\mathrm{THF})$ and $\mathrm{Pd}_{2}(\mathrm{dba})_{3}(4.6 \mathrm{mg} 0.005 \mathrm{mmol})$ were stirred at room temperature for $1 \mathrm{~h}$ and then two sample aliquots were taken and analyzed twice on GC. GC analysis of samples showed a $\mathbf{1 4} / \mathbf{1 5}$ ratio of $1.0 / 0$.

GC Data:

\begin{tabular}{l|c|c|c|c|c|}
\cline { 2 - 6 } & area naphth & area 14 & area 15 & response factor 14/15 & ratio 14/15×100 \\
\hline sample 1 & 42337 & 23484 & 0 & $0.87 / 0.79$ & $1.0 / 0$ \\
\hline sample 2 & 58993 & 33079 & 0 & $0.87 / 0.79$ & $1.0 / 0$ \\
\hline average & 50665 & 28282 & 0 & $0.87 / 0.79$ & $1.0 / 0$ \\
\hline
\end{tabular}




\section{Competition Experiments of (E)-Dimethyl-(1-pentenyl)silanol (1) vs (E)-Diphenyl-(1-} heptenyl)silanol (6) with 4-Iodoanisole. General Procedure X.
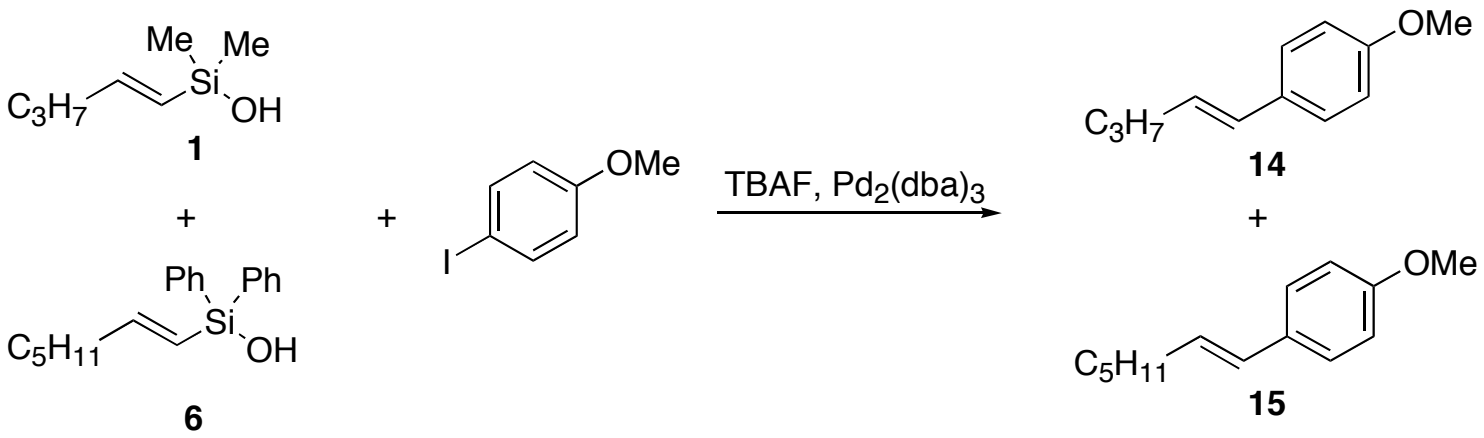

(E)-Dimethyl-(1-pentenyl)silanol (1), together with $(E)$-diphenyl-(1-heptenyl)silanol (6), naphthalene, and 4-iodoanisole were dissolved in a TBAF solution (1.0 M in THF) in a flamedried, 5-mL, 2-neck, round-bottomed flask under $\mathrm{Ar} . \mathrm{Pd}_{2}(\mathrm{dba})_{3}$ was added next and the resulting mixture stirred for $1 \mathrm{~h}$. Two $25 \mu \mathrm{L}$ samples were then taken via syringe. The sample aliquots were filtered through a plug of silica gel washing with EtOAc to achieve a total sample volume of $\sim 2 \mathrm{~mL}$. These samples were then subjected to GC analysis. Reactions were performed in duplicate.

\section{Competition of 1 equiv $(E)$-Dimethyl-(1-pentenyl)silanol (1) vs 1 equiv (E)-Diphenyl-(1- heptenyl)silanol (6) with 4-Iodoanisole and 1 equiv TBAF.}

Following General Procedure X, 1 (28.8 mg, $0.20 \mathrm{mmol}), 6$ (59.3 mg, $0.20 \mathrm{mmol})$, 4iodoanisole (46.8 mg, $0.20 \mathrm{mmol})$, naphthalene $(20.1 \mathrm{mg})$, TBAF $(0.2 \mathrm{~mL}, 0.20 \mathrm{mmol}, 1.0 \mathrm{M}$ in $\mathrm{THF})$, and $\mathrm{Pd}_{2}(\mathrm{dba})_{3}(4.6 \mathrm{mg} 0.005 \mathrm{mmol})$ were stirred at room temperature for $1 \mathrm{~h}$ and then two sample aliquots were taken and analyzed twice on GC. GC analysis of samples showed a 14/15 ratio of 42.3/57.7.

GC Data:

\begin{tabular}{l|c|c|c|c|c|}
\cline { 2 - 6 } & area naphth & area 14 & area 15 & response factor 14/15 & ratio 14/15×100 \\
\hline sample 1 & 22546 & 9143 & 14358 & $0.87 / 0.79$ & $41.3 / 58.7$ \\
\hline sample 2 & 33737 & 12703 & 18270 & $0.87 / 0.79$ & $43.4 / 56.6$ \\
\hline average & 28141 & 10923 & 16314 & $0.87 / 0.79$ & $42.3 / 57.7$ \\
\hline
\end{tabular}


Competition of 1 equiv ( $E$ )-Dimethyl-(1-pentenyl)silanol (1) vs 1 equiv (E)-Diphenyl-(1heptenyl)silanol (6) with 4-Iodoanisole and 1 equiv TBAF.

Following General Procedure X, 1 (28.8 mg, $0.20 \mathrm{mmol}), 6$ (59.3 mg, $0.20 \mathrm{mmol})$, 4iodoanisole (46.8 mg, $0.20 \mathrm{mmol})$, naphthalene $(15.9 \mathrm{mg})$, TBAF $(0.2 \mathrm{~mL}, 0.20 \mathrm{mmol}, 1.0 \mathrm{M}$ in $\mathrm{THF})$, and $\mathrm{Pd}_{2}(\mathrm{dba})_{3}(4.6 \mathrm{mg} 0.005 \mathrm{mmol})$ were stirred at room temperature for $1 \mathrm{~h}$ and then two sample aliquots were taken and analyzed twice on GC. GC analysis of samples showed a 14/15 ratio of $40.3 / 59.7$.

GC Data:

\begin{tabular}{l|c|c|c|c|c|}
\cline { 2 - 6 } & area naphth & area 14 & area 15 & response factor 14/15 & ratio 14/15×100 \\
\hline sample 1 & 17268 & 8725 & 14173 & $0.87 / 0.79$ & $40.5 / 59.5$ \\
\hline sample 2 & 13180 & 6984 & 11599 & $0.87 / 0.79$ & $40.0 / 60.0$ \\
\hline average & 15224 & 7854 & 12886 & $0.87 / 0.79$ & $40.3 / 59.7$ \\
\hline
\end{tabular}

Competition of 1 equiv (E)-Dimethyl-(1-pentenyl)silanol (1) vs 1 equiv (E)-Diphenyl-(1heptenyl)silanol (6) with 4-Iodoanisole and 2 equiv TBAF.

Following General Procedure X, 1 (28.8 mg, $0.20 \mathrm{mmol}), 6$ (59.3 mg, $0.20 \mathrm{mmol})$, 4iodoanisole $(46.8 \mathrm{mg}, 0.20 \mathrm{mmol})$, naphthalene $(20.2 \mathrm{mg})$, TBAF $(0.4 \mathrm{~mL}, 0.40 \mathrm{mmol}, 1.0 \mathrm{M}$ in $\mathrm{THF})$, and $\mathrm{Pd}_{2}(\mathrm{dba})_{3}(4.6 \mathrm{mg} 0.005 \mathrm{mmol})$ were stirred at room temperature for $1 \mathrm{~h}$ and then two sample aliquots were taken and analyzed twice on GC. GC analysis of samples showed a 14/15 ratio of 50.2/49.8.

GC Data:

\begin{tabular}{l|c|c|c|c|c|}
\cline { 2 - 6 } & area naphth & area 14 & area 15 & response factor 14/15 & ratio 14/15×100 \\
\hline sample 1 & 20967 & 12601 & 13955 & $0.87 / 0.79$ & $49.9 / 50.1$ \\
\hline sample 2 & 25207 & 14023 & 15160 & $0.87 / 0.79$ & $50.5 / 49.5$ \\
\hline average & 23087 & 13312 & 14557 & $0.87 / 0.79$ & $50.2 / 49.8$ \\
\hline
\end{tabular}

\section{Competition of 1 equiv ( $E$ )-Dimethyl-(1-pentenyl)silanol (1) vs 1 equiv (E)-Diphenyl-(1- heptenyl)silanol (6) with 4-Iodoanisole and 2 equiv TBAF.}

Following General Procedure X, 1 (28.8 mg, $0.20 \mathrm{mmol}), 6$ (59.3 mg, $0.20 \mathrm{mmol})$, 4iodoanisole $(46.8 \mathrm{mg}, 0.20 \mathrm{mmol})$, naphthalene $(15.9 \mathrm{mg})$, TBAF $(0.4 \mathrm{~mL}, 0.40 \mathrm{mmol}, 1.0 \mathrm{M}$ in $\mathrm{THF})$, and $\mathrm{Pd}_{2}(\mathrm{dba})_{3}(4.6 \mathrm{mg} 0.005 \mathrm{mmol})$ were stirred at room temperature for $1 \mathrm{~h}$ and then two 
sample aliquots were taken and analyzed twice on GC. GC analysis of samples showed a 14/15 ratio of 50.2/49.8.

GC Data:

\begin{tabular}{l|c|c|c|c|c|}
\cline { 2 - 6 } & area naphth & area 14 & area 15 & response factor 14/15 & ratio 14/15×100 \\
\hline sample 1 & 11532 & 7726 & 8375 & $0.87 / 0.79$ & $50.5 / 49.5$ \\
\hline sample 2 & 20061 & 13663 & 15283 & $0.87 / 0.79$ & $49.7 / 50.3$ \\
\hline average & 15796 & 10694 & 11829 & $0.87 / 0.79$ & $50.2 / 49.8$ \\
\hline
\end{tabular}

\section{Competition of 1 equiv $(E)$-Dimethyl-(1-pentenyl)silanol (1) vs 1 equiv (E)-Diphenyl-(1-} heptenyl)silanol (6) with 4-Iodoanisole and 4 equiv TBAF.

Following General Procedure X, 1 (28.8 mg, $0.20 \mathrm{mmol}), 6$ (59.3 mg, $0.20 \mathrm{mmol})$, 4iodoanisole (46.8 mg, $0.20 \mathrm{mmol})$, naphthalene $(19.5 \mathrm{mg})$, TBAF $(0.8 \mathrm{~mL}, 0.80 \mathrm{mmol}, 1.0 \mathrm{M}$ in $\mathrm{THF})$, and $\mathrm{Pd}_{2}(\mathrm{dba})_{3}(4.6 \mathrm{mg} 0.005 \mathrm{mmol})$ were stirred at room temperature for $1 \mathrm{~h}$ and then two sample aliquots were taken and analyzed twice on GC. GC analysis of samples showed a 14/15 ratio of 59.5/40.5.

GC Data:

\begin{tabular}{l|c|c|c|c|c|}
\cline { 2 - 6 } & area naphth & area 14 & area 15 & response factor 14/15 & ratio 14/15×100 \\
\hline sample 1 & 12345 & 7335 & 5487 & $0.87 / 0.79$ & $59.6 / 40.4$ \\
\hline sample 2 & 16274 & 10275 & 7769 & $0.87 / 0.79$ & $59.4 / 40.6$ \\
\hline average & 14309 & 8805 & 6628 & $0.87 / 0.79$ & $59.5 / 40.5$ \\
\hline
\end{tabular}

Competition of 1 equiv $(E)$-Dimethyl-(1-pentenyl)silanol (1) vs 1 equiv (E)-Diphenyl-(1heptenyl)silanol (6) with 4-Iodoanisole and 4 equiv TBAF.

Following General Procedure X, 1 (28.8 mg, $0.20 \mathrm{mmol}), 6$ (59.3 mg, $0.20 \mathrm{mmol})$, 4iodoanisole $(46.8 \mathrm{mg}, 0.20 \mathrm{mmol})$, naphthalene $(15.9 \mathrm{mg})$, TBAF $(0.8 \mathrm{~mL}, 0.80 \mathrm{mmol}, 1.0 \mathrm{M}$ in $\mathrm{THF})$, and $\mathrm{Pd}_{2}(\mathrm{dba})_{3}(4.6 \mathrm{mg} 0.005 \mathrm{mmol})$ were stirred at room temperature for $1 \mathrm{~h}$ and then two sample aliquots were taken and analyzed twice on GC. GC analysis of samples showed a 14/15 ratio of 59.8/40.2. 
GC Data:

\begin{tabular}{l|c|c|c|c|c|}
\cline { 2 - 6 } & area naphth & area 14 & area 15 & response factor 14/15 & ratio 14/15×100 \\
\hline sample 1 & 23124 & 21653 & 16187 & $0.87 / 0.79$ & $59.6 / 40.4$ \\
\hline sample 2 & 27174 & 16805 & 12354 & $0.87 / 0.79$ & $60.0 / 40.0$ \\
\hline average & 25149 & 19229 & 14270 & $0.87 / 0.79$ & $59.8 / 40.2$ \\
\hline
\end{tabular}

\section{Competition of 1 equiv (E)-Dimethyl-(1-pentenyl)silanol (1) vs 1 equiv (E)-Diphenyl-(1-} heptenyl)silanol (6) with 4-Iodoanisole and 8 equiv TBAF.

Following General Procedure X, 1 (28.8 mg, $0.20 \mathrm{mmol}), 6$ (59.3 mg, $0.20 \mathrm{mmol})$, 4iodoanisole $(46.8 \mathrm{mg}, 0.20 \mathrm{mmol})$, naphthalene $(25.4 \mathrm{mg})$, TBAF $(1.6 \mathrm{~mL}, 1.60 \mathrm{mmol}, 1.0 \mathrm{M}$ in $\mathrm{THF})$, and $\mathrm{Pd}_{2}(\mathrm{dba})_{3}(4.6 \mathrm{mg} 0.005 \mathrm{mmol})$ were stirred at room temperature for $1 \mathrm{~h}$ and then two sample aliquots were taken and analyzed twice on GC. GC analysis of samples showed a 14/15 ratio of $50.5 / 49.5$.

GC Data:

\begin{tabular}{l|c|c|c|c|c|}
\cline { 2 - 6 } & area naphth & area 14 & area 15 & response factor 14/15 & ratio 14/15×100 \\
\hline sample 1 & 17080 & 6780 & 6930 & $0.87 / 0.79$ & $51.9 / 48.1$ \\
\hline sample 2 & 13917 & 5536 & 6292 & $0.87 / 0.79$ & $49.3 / 50.7$ \\
\hline average & 15498 & 6158 & 6611 & $0.87 / 0.79$ & $50.5 / 49.5$ \\
\hline
\end{tabular}

\section{Competition of 1 equiv (E)-Dimethyl-(1-pentenyl)silanol (1) vs 1 equiv (E)-Diphenyl-(1-} heptenyl)silanol (6) with 4-Iodoanisole and 8 equiv TBAF.

Following General Procedure X, 1 (28.8 mg, $0.20 \mathrm{mmol}), 6$ (59.3 mg, $0.20 \mathrm{mmol})$, 4iodoanisole $(46.8 \mathrm{mg}, 0.20 \mathrm{mmol})$, naphthalene $(15.9 \mathrm{mg})$, TBAF (1.6 mL, 1.60 mmol, 1.0 M in $\mathrm{THF})$, and $\mathrm{Pd}_{2}(\mathrm{dba})_{3}(4.6 \mathrm{mg} 0.005 \mathrm{mmol})$ were stirred at room temperature for $1 \mathrm{~h}$ and then two sample aliquots were taken and analyzed twice on GC. GC analysis of samples showed a 14/15 ratio of $49.5 / 50.5$.

GC Data:

\begin{tabular}{l|c|c|c|c|c|}
\cline { 2 - 5 } & area naphth & area 14 & area 15 & response factor 14/15 & ratio 14/15×100 \\
\hline sample 1 & 9853 & 6193 & 7086 & $0.87 / 0.79$ & $49.1 / 50.9$ \\
\hline sample 2 & 9333 & 6034 & 6722 & $0.87 / 0.79$ & $49.8 / 50.2$ \\
\hline average & 9593 & 6113 & 6904 & $0.87 / 0.79$ & $49.5 / 50.5$ \\
\hline
\end{tabular}




\section{Competition of 2 equiv (E)-Dimethyl-(1-pentenyl)silanol (1) vs 2 equiv (E)-Diphenyl-(1-} heptenyl)silanol (6) with 4-Iodoanisole and 8 equiv TBAF.

Following General Procedure X, 1 (28.8 mg, $0.20 \mathrm{mmol}), 6$ (59.3 mg, $0.20 \mathrm{mmol})$, 4iodoanisole $(23.4 \mathrm{mg}, 0.10 \mathrm{mmol})$, naphthalene $(10.8 \mathrm{mg})$, TBAF $(0.8 \mathrm{~mL}, 0.8 \mathrm{mmol}, 1.0 \mathrm{M}$ in $\mathrm{THF})$, and $\mathrm{Pd}_{2}(\mathrm{dba})_{3}(2.3 \mathrm{mg} 0.0025 \mathrm{mmol})$ were stirred at room temperature for $1 \mathrm{~h}$ and then two sample aliquots were taken and analyzed twice on GC. GC analysis of samples showed a $\mathbf{1 4 / 1 5}$ ratio of $59.4 / 40.6$.

GC Data:

\begin{tabular}{l|c|c|c|c|c|}
\cline { 2 - 5 } & area naphth & area 14 & area 15 & response factor 14/15 & ratio 14/15×100 \\
\hline sample 1 & 11521 & 5999 & 4543 & $0.87 / 0.79$ & $59.3 / 40.7$ \\
\hline sample 2 & 13250 & 6885 & 5197 & $0.87 / 0.79$ & $59.4 / 40.6$ \\
\hline average & 12385 & 6442 & 4847 & $0.87 / 0.79$ & $59.4 / 40.6$ \\
\hline
\end{tabular}

Competition of 2 equiv (E)-Dimethyl-(1-pentenyl)silanol (1) vs 2 equiv (E)-Diphenyl-(1heptenyl)silanol (6) with 4-Iodoanisole and 8 equiv TBAF.

Following General Procedure X, 1 (28.8 mg, $0.20 \mathrm{mmol}), 6$ (59.3 mg, $0.20 \mathrm{mmol})$, 4iodoanisole (23.4 mg, $0.10 \mathrm{mmol})$, naphthalene $(15.9 \mathrm{mg})$, TBAF $(0.8 \mathrm{~mL}, 0.8 \mathrm{mmol}, 1.0 \mathrm{M}$ in $\mathrm{THF})$, and $\mathrm{Pd}_{2}(\mathrm{dba})_{3}(2.3 \mathrm{mg} 0.0025 \mathrm{mmol})$ were stirred at room temperature for $1 \mathrm{~h}$ and then two sample aliquots were taken and analyzed twice on GC. GC analysis of samples showed a $\mathbf{1 4} / \mathbf{1 5}$ ratio of $59.9 / 40.1$.

GC Data:

\begin{tabular}{l|c|c|c|c|c|}
\cline { 2 - 5 } & area naphth & area 14 & area 15 & response factor 14/15 & ratio 14/15×100 \\
\hline sample 1 & 8203 & 8043 & 5865 & $0.87 / 0.79$ & $60.2 / 39.8$ \\
\hline sample 2 & 5103 & 4599 & 3444 & $0.87 / 0.79$ & $59.6 / 40.4$ \\
\hline average & 6653 & 4821 & 4654 & $0.87 / 0.79$ & $59.9 / 40.1$ \\
\hline
\end{tabular}

\section{Competition of 3 equiv (E)-Dimethyl-(1-pentenyl)silanol (1) vs 3 equiv (E)-Diphenyl-(1-} heptenyl)silanol (6) with 4-Iodoanisole and 12 equiv TBAF.

Following General Procedure X, 1 (43.3 mg, $0.30 \mathrm{mmol}), 6$ (88.9 mg, $0.30 \mathrm{mmol})$, 4iodoanisole $(23.4 \mathrm{mg}, 0.10 \mathrm{mmol})$, naphthalene $(15.9 \mathrm{mg})$, TBAF (1.2 mL, 1.2 mmol, 1.0 M in $\mathrm{THF})$, and $\mathrm{Pd}_{2}(\mathrm{dba})_{3}(2.3 \mathrm{mg} 0.0025 \mathrm{mmol})$ were stirred at room temperature for $1 \mathrm{~h}$ and then 
two sample aliquots were taken and analyzed twice on GC. GC analysis of samples showed a $\mathbf{1 4} / \mathbf{1 5}$ ratio of $58.8 / 41.2$.

GC Data:

\begin{tabular}{l|c|c|c|c|c|}
\cline { 2 - 5 } & area naphth & area 14 & area 15 & response factor 14/15 & ratio 14/15×100 \\
\hline sample 1 & 9551 & 4979 & 3736 & $0.87 / 0.79$ & $59.6 / 40.4$ \\
\hline sample 2 & 7102 & 6100 & 4907 & $0.87 / 0.79$ & $57.9 / 42.1$ \\
\hline average & 8326 & 5539 & 4321 & $0.87 / 0.79$ & $58.8 / 41.2$ \\
\hline
\end{tabular}

\section{Competition of 3 equiv (E)-Dimethyl-(1-pentenyl)silanol (1) vs 3 equiv (E)-Diphenyl-(1- heptenyl)silanol (6) with 4-Iodoanisole and 12 equiv TBAF.}

Following General Procedure X, 1 (43.3 mg, $0.30 \mathrm{mmol}), 6$ (88.9 mg, $0.30 \mathrm{mmol})$, 4iodoanisole (23.4 mg, $0.10 \mathrm{mmol})$, naphthalene (13.6 mg), TBAF (1.2 mL, 1.2 mmol, 1.0 M in $\mathrm{THF})$, and $\mathrm{Pd}_{2}(\mathrm{dba})_{3}(2.3 \mathrm{mg} 0.0025 \mathrm{mmol})$ were stirred at room temperature for $1 \mathrm{~h}$ and then two sample aliquots were taken and analyzed twice on GC. GC analysis of samples showed a $\mathbf{1 4 / 1 5}$ ratio of $57.7 / 42.3$.

GC Data:

\begin{tabular}{l|c|c|c|c|c|}
\cline { 2 - 5 } & area naphth & area 14 & area 15 & response factor 14/15 & ratio 14/15×100 \\
\hline sample 1 & 12218 & 5908 & 4766 & $0.87 / 0.79$ & $57.8 / 42.2$ \\
\hline sample 2 & 14495 & 6549 & 5322 & $0.87 / 0.79$ & $57.6 / 42.4$ \\
\hline average & 13356 & 6228 & 5044 & $0.87 / 0.79$ & $57.7 / 42.3$ \\
\hline
\end{tabular}

\section{Competition of 1 equiv $(E)$-Dimethyl-(1-pentenyl)silanol (1) vs 1 equiv (E)-Diphenyl-(1-} heptenyl)silanol (6) with 4-Iodoanisole and 1 equiv TMSOK.

Following General Procedure X, 1 (28.8 mg, $0.20 \mathrm{mmol}), 6$ (59.3 mg, $0.20 \mathrm{mmol})$, 4iodoanisole (46.8 mg, $0.20 \mathrm{mmol}$ ), naphthalene (18.1 mg), TMSOK (25.6 mg, $0.20 \mathrm{mmol})$, THF $(0.4 \mathrm{~mL})$ and $\mathrm{Pd}_{2}(\mathrm{dba})_{3}(4.6 \mathrm{mg} 0.005 \mathrm{mmol})$ were stirred at room temperature for $1 \mathrm{~h}$ and then two sample aliquots were taken and analyzed twice on GC. GC analysis of samples showed a $\mathbf{1 4} / \mathbf{1 5}$ ratio of $15.8 / 84.2$. 
GC Data:

\begin{tabular}{l|c|c|c|c|c|}
\cline { 2 - 6 } & area naphth & area 14 & area 15 & response factor 14/15 & ratio 14/15×100 \\
\hline sample 1 & 28110 & 1051 & 6006 & $0.87 / 0.79$ & $16.2 / 83.8$ \\
\hline sample 2 & 33532 & 1120 & 6827 & $0.87 / 0.79$ & $15.3 / 84.7$ \\
\hline average & 30821 & 1085 & 6416 & $0.87 / 0.79$ & $15.8 / 84.2$ \\
\hline
\end{tabular}

Competition of 1 equiv $(E)$-Dimethyl-(1-pentenyl)silanol (1) vs 1 equiv (E)-Diphenyl-(1heptenyl)silanol (6) with 4-Iodoanisole and 1 equiv TMSOK.

Following General Procedure X, 1 (28.8 mg, $0.20 \mathrm{mmol}), 6$ (59.3 mg, $0.20 \mathrm{mmol})$, 4iodoanisole (46.8 mg, $0.20 \mathrm{mmol}$ ), naphthalene (15.9 mg), TMSOK (25.6 mg, $0.20 \mathrm{mmol})$, THF $(0.4 \mathrm{~mL})$ and $\mathrm{Pd}_{2}(\mathrm{dba})_{3}(4.6 \mathrm{mg} 0.005 \mathrm{mmol})$ were stirred at room temperature for $1 \mathrm{~h}$ and then two sample aliquots were taken and analyzed twice on GC. GC analysis of samples showed a $\mathbf{1 4} / \mathbf{1 5}$ ratio of $12.5 / 87.5$.

GC Data:

\begin{tabular}{l|c|c|c|c|c|}
\cline { 2 - 6 } & area naphth & area 14 & area 15 & response factor 14/15 & ratio 14/15×100 \\
\hline sample 1 & 26560 & 992 & 7692 & $0.87 / 0.79$ & $12.5 / 87.5$ \\
\hline sample 2 & 14063 & 509 & 3952 & $0.87 / 0.79$ & $12.5 / 87.5$ \\
\hline average & 20311 & 750 & 5822 & $0.87 / 0.79$ & $12.5 / 87.5$ \\
\hline
\end{tabular}

\section{Competition of 1 equiv ( $E$ )-Dimethyl-(1-pentenyl)silanol (1) vs 1 equiv (E)-Diphenyl-(1- heptenyl)silanol (6) with 4-Iodoanisole and 2 equiv TMSOK.}

Following General Procedure X, 1 (28.8 mg, $0.20 \mathrm{mmol}), 6$ (59.3 mg, $0.20 \mathrm{mmol})$, 4iodoanisole (46.8 mg, $0.20 \mathrm{mmol}$ ), naphthalene (15.9 mg), TMSOK (51.2 mg, $0.40 \mathrm{mmol})$, THF $(0.4 \mathrm{~mL})$ and $\mathrm{Pd}_{2}(\mathrm{dba})_{3}(4.6 \mathrm{mg} 0.005 \mathrm{mmol})$ were stirred at room temperature for $1 \mathrm{~h}$ and then two sample aliquots were taken and analyzed twice on GC. GC analysis of samples showed a $\mathbf{1 4 / 1 5}$ ratio of $15.7 / 84.3$.

GC Data:

\begin{tabular}{l|c|c|c|c|c|}
\cline { 2 - 5 } & area naphth & area 14 & area 15 & response factor 14/15 & ratio 14/15×100 \\
\hline sample 1 & 27147 & 3535 & 20885 & $0.87 / 0.79$ & $15.8 / 84.2$ \\
\hline sample 2 & 37987 & 5169 & 30850 & $0.87 / 0.79$ & $15.6 / 84.4$ \\
\hline average & 32567 & 4352 & 25867 & $0.87 / 0.79$ & $15.7 / 84.3$ \\
\hline
\end{tabular}


Competition of 1 equiv (E)-Dimethyl-(1-pentenyl)silanol (1) vs 1 equiv $(E)$-Diphenyl-(1heptenyl)silanol (6) with 4-Iodoanisole and 2 equiv TMSOK.

Following General Procedure X, 1 (28.8 mg, $0.20 \mathrm{mmol}), 6$ (59.3 mg, $0.20 \mathrm{mmol})$, 4iodoanisole (46.8 mg, $0.20 \mathrm{mmol}$ ), naphthalene (19.6 mg), TMSOK (51.2 mg, $0.40 \mathrm{mmol}$ ), THF $(0.4 \mathrm{~mL})$ and $\mathrm{Pd}_{2}(\mathrm{dba})_{3}(4.6 \mathrm{mg} 0.005 \mathrm{mmol})$ were stirred at room temperature for $1 \mathrm{~h}$ and then two sample aliquots were taken and analyzed twice on GC. GC analysis of samples showed a $\mathbf{1 4 / 1 5}$ ratio of $16.3 / 83.7$.

GC Data:

\begin{tabular}{l|c|c|c|c|c|}
\cline { 2 - 6 } & area naphth & area 14 & area 15 & response factor 14/15 & ratio 14/15×100 \\
\hline sample 1 & 37643 & 4556 & 25154 & $0.87 / 0.79$ & $16.7 / 83.3$ \\
\hline sample 2 & 30725 & 3506 & 20405 & $0.87 / 0.79$ & $16.0 / 84.0$ \\
\hline average & 34184 & 4031 & 22779 & $0.87 / 0.79$ & $16.3 / 83.7$ \\
\hline
\end{tabular}

\section{Competition of 1 equiv (E)-Dimethyl-(1-pentenyl)silanol (1) vs 1 equiv (E)-Diphenyl-(1-} heptenyl)silanol (6) with 4-Iodoanisole and 4 equiv TMSOK.

Following General Procedure X, 1 (28.8 mg, $0.20 \mathrm{mmol}), 6$ (59.3 mg, $0.20 \mathrm{mmol})$, 4iodoanisole (46.8 mg, $0.20 \mathrm{mmol}$ ), naphthalene (20.9 mg), TMSOK (103 mg, $0.80 \mathrm{mmol}$ ), THF $(0.8 \mathrm{~mL})$ and $\mathrm{Pd}_{2}(\mathrm{dba})_{3}(4.6 \mathrm{mg} 0.005 \mathrm{mmol})$ were stirred at room temperature for $1 \mathrm{~h}$ and then two sample aliquots were taken and analyzed twice on GC. GC analysis of samples showed a $\mathbf{1 4} / \mathbf{1 5}$ ratio of $40.2 / 59.8$.

GC Data:

\begin{tabular}{l|c|c|c|c|c|}
\cline { 2 - 6 } & area naphth & area 14 & area 15 & response factor 14/15 & ratio 14/15×100 \\
\hline sample 1 & 20175 & 7329 & 12366 & $0.87 / 0.79$ & $39.6 / 60.4$ \\
\hline sample 2 & 19947 & 7353 & 11844 & $0.87 / 0.79$ & $40.7 / 59.3$ \\
\hline average & 20061 & 7341 & 12105 & $0.87 / 0.79$ & $40.2 / 59.8$ \\
\hline
\end{tabular}

\section{Competition of 1 equiv (E)-Dimethyl-(1-pentenyl)silanol (1) vs 1 equiv (E)-Diphenyl-(1-} heptenyl)silanol (6) with 4-Iodoanisole and 4 equiv TMSOK.

Following General Procedure X, 1 (28.8 mg, $0.20 \mathrm{mmol}), 6$ (59.3 mg, $0.20 \mathrm{mmol})$, 4iodoanisole (46.8 mg, $0.20 \mathrm{mmol}$ ), naphthalene (15.9 mg), TMSOK (103 mg, $0.80 \mathrm{mmol})$, THF $(0.8 \mathrm{~mL})$ and $\mathrm{Pd}_{2}(\mathrm{dba})_{3}(4.6 \mathrm{mg} 0.005 \mathrm{mmol})$ were stirred at room temperature for $1 \mathrm{~h}$ and then 
two sample aliquots were taken and analyzed twice on GC. GC analysis of samples showed a $\mathbf{1 4} / \mathbf{1 5}$ ratio of $31.4 / 68.6$.

GC Data:

\begin{tabular}{l|c|c|c|c|c|}
\cline { 2 - 6 } & area naphth & area 14 & area 15 & response factor 14/15 & ratio 14/15×100 \\
\hline sample 1 & 14843 & 6020 & 14238 & $0.87 / 0.79$ & $31.8 / 68.2$ \\
\hline sample 2 & 16122 & 6836 & 16818 & $0.87 / 0.79$ & $31.0 / 69.0$ \\
\hline average & 15482 & 6428 & 15528 & $0.87 / 0.79$ & $31.4 / 68.6$ \\
\hline
\end{tabular}

\section{Competition of 1 equiv (E)-Dimethyl-(1-pentenyl)silanol (1) vs 1 equiv (E)-Diphenyl-(1-} heptenyl)silanol (6) with 4-Iodoanisole and 4 equiv TMSOK.

Following General Procedure X, 1 (28.8 mg, $0.20 \mathrm{mmol}), 6$ (59.3 mg, $0.20 \mathrm{mmol})$, 4iodoanisole (46.8 mg, $0.20 \mathrm{mmol})$, naphthalene (13.1 mg), TMSOK (103 mg, $0.80 \mathrm{mmol})$, THF $(0.8 \mathrm{~mL})$ and $\mathrm{Pd}_{2}(\mathrm{dba})_{3}(4.6 \mathrm{mg} 0.005 \mathrm{mmol})$ were stirred at room temperature for $1 \mathrm{~h}$ and then two sample aliquots were taken and analyzed twice on GC. GC analysis of samples showed a $\mathbf{1 4} / \mathbf{1 5}$ ratio of $35.2 / 64.8$.

GC Data:

\begin{tabular}{l|c|c|c|c|c|}
\cline { 2 - 5 } & area naphth & area 14 & area 15 & response factor 14/15 & ratio 14/15×100 \\
\hline sample 1 & 16624 & 9111 & 16841 & $0.87 / 0.79$ & $37.4 / 62.6$ \\
\hline sample 2 & 18291 & 9833 & 22055 & $0.87 / 0.79$ & $33.0 / 67.0$ \\
\hline average & 17457 & 9472 & 19448 & $0.87 / 0.79$ & $35.2 / 64.8$ \\
\hline
\end{tabular}

\section{Competition of 1 equiv (E)-Dimethyl-(1-pentenyl)silanol (1) vs 1 equiv (E)-Diphenyl-(1-} heptenyl)silanol (6) with 4-Iodoanisole and 8 equiv TMSOK.

Following General Procedure X, 1 (28.8 mg, $0.20 \mathrm{mmol}), 6$ (59.3 mg, $0.20 \mathrm{mmol})$, 4iodoanisole (46.8 mg, $0.20 \mathrm{mmol}$ ), naphthalene (20.1 mg), TMSOK (205 mg, $1.60 \mathrm{mmol})$, THF $(1.6 \mathrm{~mL})$ and $\mathrm{Pd}_{2}(\mathrm{dba})_{3}(4.6 \mathrm{mg} 0.005 \mathrm{mmol})$ were stirred at room temperature for $1 \mathrm{~h}$ and then two sample aliquots were taken and analyzed twice on GC. GC analysis of samples showed a $\mathbf{1 4 / 1 5}$ ratio of $61.2 / 38.8$. 
GC Data:

\begin{tabular}{l|c|c|c|c|c|}
\cline { 2 - 5 } & area naphth & area 14 & area 15 & response factor 14/15 & ratio 14/15×100 \\
\hline sample 1 & 9806 & 6231 & 4376 & $0.87 / 0.79$ & $61.1 / 38.9$ \\
\hline sample 2 & 7811 & 4701 & 3283 & $0.87 / 0.79$ & $61.3 / 38.7$ \\
\hline average & 8808 & 5466 & 3829 & $0.87 / 0.79$ & $61.2 / 38.8$ \\
\hline
\end{tabular}

Competition of 1 equiv $(E)$-Dimethyl-(1-pentenyl)silanol (1) vs 1 equiv (E)-Diphenyl-(1heptenyl)silanol (6) with 4-Iodoanisole and 8 equiv TMSOK.

Following General Procedure X, 1 (28.8 mg, $0.20 \mathrm{mmol}), 6$ (59.3 mg, $0.20 \mathrm{mmol})$, 4iodoanisole (46.8 mg, $0.20 \mathrm{mmol}$ ), naphthalene (15.9 mg), TMSOK (205 mg, $1.60 \mathrm{mmol}$ ), THF $(1.6 \mathrm{~mL})$ and $\mathrm{Pd}_{2}(\mathrm{dba})_{3}(4.6 \mathrm{mg} 0.005 \mathrm{mmol})$ were stirred at room temperature for $1 \mathrm{~h}$ and then two sample aliquots were taken and analyzed twice on GC. GC analysis of samples showed a $\mathbf{1 4} / \mathbf{1 5}$ ratio of $56.4 / 43.6$.

GC Data:

\begin{tabular}{l|c|c|c|c|c|}
\cline { 2 - 5 } & area naphth & area 14 & area 1 5 & response factor 14/15 & ratio 14/15x100 \\
\hline sample 1 & 6228 & 2407 & 1909 & $0.87 / 0.79$ & $58.2 / 41.8$ \\
\hline sample 2 & 5060 & 2138 & 1971 & $0.87 / 0.79$ & $54.5 / 45.5$ \\
\hline average & 5644 & 2272 & 1940 & $0.87 / 0.79$ & $56.4 / 43.6$ \\
\hline
\end{tabular}

\section{Competition of 2 equiv (E)-Dimethyl-(1-pentenyl)silanol (1) vs 2 equiv (E)-Diphenyl-(1- heptenyl)silanol (6) with 4-Iodoanisole and 8 equiv TMSOK.}

Following General Procedure X, 1 (28.8 mg, $0.20 \mathrm{mmol}), 6$ (59.3 mg, $0.20 \mathrm{mmol})$, 4iodoanisole (23.4 mg, $0.10 \mathrm{mmol}$ ), naphthalene (11.9 mg), TMSOK (103 mg, $0.80 \mathrm{mmol})$, THF $(0.8 \mathrm{~mL})$ and $\mathrm{Pd}_{2}(\mathrm{dba})_{3}(2.3 \mathrm{mg} 0.0025 \mathrm{mmol})$ were stirred at room temperature for $1 \mathrm{~h}$ and then two sample aliquots were taken and analyzed twice on GC. GC analysis of samples showed a $\mathbf{1 4 / 1 5}$ ratio of $35.7 / 64.3$.

GC Data:

\begin{tabular}{l|c|c|c|c|c|}
\cline { 2 - 6 } & area naphth & area 14 & area 15 & response factor 14/15 & ratio 14/15×100 \\
\hline sample 1 & 11054 & 3523 & 7644 & $0.87 / 0.79$ & $33.7 / 66.3$ \\
\hline sample 2 & 12260 & 4103 & 7514 & $0.87 / 0.79$ & $37.6 / 62.4$ \\
\hline average & 11657 & 3813 & 7579 & $0.87 / 0.79$ & $35.7 / 64.3$ \\
\hline
\end{tabular}


Competition of 2 equiv ( $E$ )-Dimethyl-(1-pentenyl)silanol (1) vs 2 equiv (E)-Diphenyl-(1heptenyl)silanol (6) with 4-Iodoanisole and 8 equiv TMSOK.

Following General Procedure X, 1 (28.8 mg, $0.20 \mathrm{mmol}), 6$ (59.3 mg, $0.20 \mathrm{mmol})$, 4iodoanisole (23.4 mg, $0.10 \mathrm{mmol})$, naphthalene $(15.9 \mathrm{mg})$, TMSOK (103 mg, $0.80 \mathrm{mmol})$, THF $(0.8 \mathrm{~mL})$ and $\mathrm{Pd}_{2}(\mathrm{dba})_{3}(2.3 \mathrm{mg} 0.0025 \mathrm{mmol})$ were stirred at room temperature for $1 \mathrm{~h}$ and then two sample aliquots were taken and analyzed twice on GC. GC analysis of samples showed a $\mathbf{1 4} / \mathbf{1 5}$ ratio of $33.5 / 66.5$.

GC Data:

\begin{tabular}{l|c|c|c|c|c|}
\cline { 2 - 5 } & area naphth & area 14 & area 15 & response factor 14/15 & ratio 14/15×100 \\
\hline sample 1 & 6176 & 2685 & 5603 & $0.87 / 0.79$ & $34.6 / 65.4$ \\
\hline sample 2 & 5039 & 2091 & 4827 & $0.87 / 0.79$ & $32.4 / 67.6$ \\
\hline average & 5607 & 2388 & 5215 & $0.87 / 0.79$ & $33.5 / 66.5$ \\
\hline
\end{tabular}

\section{Competition of 3 equiv (E)-Dimethyl-(1-pentenyl)silanol (1) vs 3 equiv (E)-Diphenyl-(1-} heptenyl)silanol (6) with 4-Iodoanisole and 12 equiv TMSOK.

Following General Procedure X, 1 (43.3 mg, $0.30 \mathrm{mmol}), 6$ (88.9 mg, $0.20 \mathrm{mmol})$, 4iodoanisole (23.4 mg, $0.10 \mathrm{mmol})$, naphthalene (14.0 mg), TMSOK (103 mg, $0.80 \mathrm{mmol})$, THF $(0.8 \mathrm{~mL})$ and $\mathrm{Pd}_{2}(\mathrm{dba})_{3}(2.3 \mathrm{mg} 0.0025 \mathrm{mmol})$ were stirred at room temperature for $1 \mathrm{~h}$ and then two sample aliquots were taken and analyzed twice on GC. GC analysis of samples showed a $\mathbf{1 4} / \mathbf{1 5}$ ratio of $39.6 / 60.4$.

GC Data:

\begin{tabular}{l|c|c|c|c|c|}
\cline { 2 - 6 } & area naphth & area 14 & area 15 & response factor 14/15 & ratio 14/15×100 \\
\hline sample 1 & 7584 & 2232 & 3655 & $0.87 / 0.79$ & $40.3 / 59.7$ \\
\hline sample 2 & 8436 & 2222 & 3845 & $0.87 / 0.79$ & $39.0 / 61.0$ \\
\hline average & 8010 & 2227 & 3750 & $0.87 / 0.79$ & $39.6 / 60.4$ \\
\hline
\end{tabular}

\section{Competition of 3 equiv (E)-Dimethyl-(1-pentenyl)silanol (1) vs 3 equiv (E)-Diphenyl-(1-} heptenyl)silanol (6) with 4-Iodoanisole and 12 equiv TMSOK.

Following General Procedure X, 1 (43.3 mg, $0.30 \mathrm{mmol}), 6$ (88.9 mg, $0.20 \mathrm{mmol})$, 4iodoanisole (23.4 mg, $0.10 \mathrm{mmol})$, naphthalene (15.9 mg), TMSOK (103 mg, $0.80 \mathrm{mmol})$, THF $(0.8 \mathrm{~mL})$ and $\mathrm{Pd}_{2}(\mathrm{dba})_{3}(2.3 \mathrm{mg} 0.0025 \mathrm{mmol})$ were stirred at room temperature for $1 \mathrm{~h}$ and then 
two sample aliquots were taken and analyzed twice on GC. GC analysis of samples showed a $\mathbf{1 4} / \mathbf{1 5}$ ratio of $36.2 / 63.8$.

GC Data:

\begin{tabular}{l|c|c|c|c|c|}
\cline { 2 - 6 } & area naphth & area 14 & area 15 & response factor 14/15 & ratio 14/15×100 \\
\hline sample 1 & 4874 & 1863 & 3955 & $0.87 / 0.79$ & $34.2 / 65.8$ \\
\hline sample 2 & 4703 & 2188 & 3917 & $0.87 / 0.79$ & $38.2 / 61.8$ \\
\hline average & 4788 & 2025 & 3936 & $0.87 / 0.79$ & $36.2 / 63.8$ \\
\hline
\end{tabular}

\section{References}

(1) Gilman, H.; Cartledge, F. K.; Sin, S.-Y. J. Organomet. Chem. 1963, 1, 8.

(2) Chandra, G.; Lo, P. Y.; Hitencock, P. B.; Lappert, M. F. Organometallics 1987, 6, 191.

(3) Denmark, S. E.; Wehrli, D.; Choi, J. Y. Org. Lett. 2000, 2, 2491.

(4) Barrett, A. G. M.; Bennett, A. J.; Menzer, S.; Smith, M. L.; White, A. J. P.; Williams, D. J. J. Org. Chem. 1999, 64, 162.

(5) Le Bigot, Y.; Delmas, M.; Gaset, A. Synth. Commun. 1982, 12, 1115.

(6) Austin, W. B.; Bilow, N.; Kelleghan, J.; Lau, J. S. Y. J. Org. Chem. 1981, 46, 2280.

(7) Esteruelas M. A.; Werner, H. J. Organomet. Chem. 1986, 303, 221.

(8) Tsuda, K.; Ishizone, T.; Hirao, A.; Nakahama, S. Macromolecules 1993, 26, 6985.

(9) Meng, H.-H. B.; Dalton, L. R.; Wu, S.-T. Mol. Cryst. Liq. Cryst. Sci. Technol. Sect. A 1994, 250, 303.

(10) Stammel, C.; Frohlich, R.; Wolf, C.; Wenck, H.; de Meijere, A.; Mattay, J. Eur. J. Org. Chem. 1999, 1790. 